Review

\title{
Fossil History of Curculionoidea (Coleoptera) from the Paleogene
}

\author{
Andrei A. Legalov 1,2 (1) \\ 1 Institute of Systematics and Ecology of Animals, Siberian Branch, Russian Academy of Sciences, Ulitsa \\ Frunze, 11, 630091 Novosibirsk, Novosibirsk Oblast, Russia; fossilweevils@gmail.com; Tel.: +7-9139471413 \\ 2 Biological Institute, Tomsk State University, Lenin Ave, 36, 634050 Tomsk, Tomsk Oblast, Russia
}

Received: 23 June 2020; Accepted: 4 September 2020; Published: 6 September 2020

check for updates

\begin{abstract}
Currently, some 564 species of Curculionoidea from nine families (Nemonychidae-4, Anthribidae-33, Ithyceridae-3, Belidae-9, Rhynchitidae-41, Attelabidae-3, Brentidae-47, Curculionidae-384, Platypodidae-2, Scolytidae-37) are known from the Paleogene. Twenty-seven species are found in the Paleocene, 442 in the Eocene and 94 in the Oligocene. The greatest diversity of Curculionoidea is described from the Eocene of Europe and North America. The richest faunas are known from Eocene localities, Florissant (177 species), Baltic amber (124 species) and Green River formation (75 species). The family Curculionidae dominates in all Paleogene localities. Weevil species associated with herbaceous vegetation are present in most localities since the middle Paleocene. A list of Curculionoidea species and their distribution by location is presented.
\end{abstract}

Keywords: Coleoptera; Curculionoidea; fossil weevil; faunal structure; Paleocene; Eocene; Oligocene

\section{Introduction}

Research into the biodiversity of the past is very important for understanding the development of life on our planet. Insects are one of the Main components of both extinct and recent ecosystems. Coleoptera occupied a special place in the terrestrial animal biotas of the Mesozoic and Cenozoics, as they are characterized by not only great diversity but also by their ecological specialization. The largest superfamily in the Coleoptera is the Curculionoidea, which, among beetles, is one of the Main plant consumers. They develop in living or dead tissues of various plant organs as well as in soil, feeding on roots. As an exception, some weevil larvae can develop completely exposed on plants, as well as on the surface of the soil as detritivores or predators.

The purpose of this study is to evaluate the diversity of Curculionoidea in the Paleogene and to show the specificity of faunas in various epochs, ages and localities. This is the first time a review of the Paleogene Curculionoid beetle has been undertaken.

\section{Materials and Methods}

The Paleogene is the first period of the Cenozoic, consisting of three epochs, the Paleocene, Eocene and Oligocene (Figure 1). The Paleogene started at about $66 \mathrm{Ma}$ and ended around $23 \mathrm{Ma}$. The Late Cretaceous preceded it. The Miocene (from the Neogene) began after the Paleogene. The Paleocene consists of three ages (Danian, Selandian and Thanetian). The Eocene is subdivided into three subepochs (Early, Middle and Late) with four ages (Ypresian, Lutetian, Bartonian and Priabonian). The Bridgerian stands out for the North American Provincial Ages at the end of the Ypresian and the beginning of the Lutetian. This age corresponds with the Green River Formation. The Oligocene consists of two subepochs (Early and Late) and two ages (Rupelian and Chattian). 


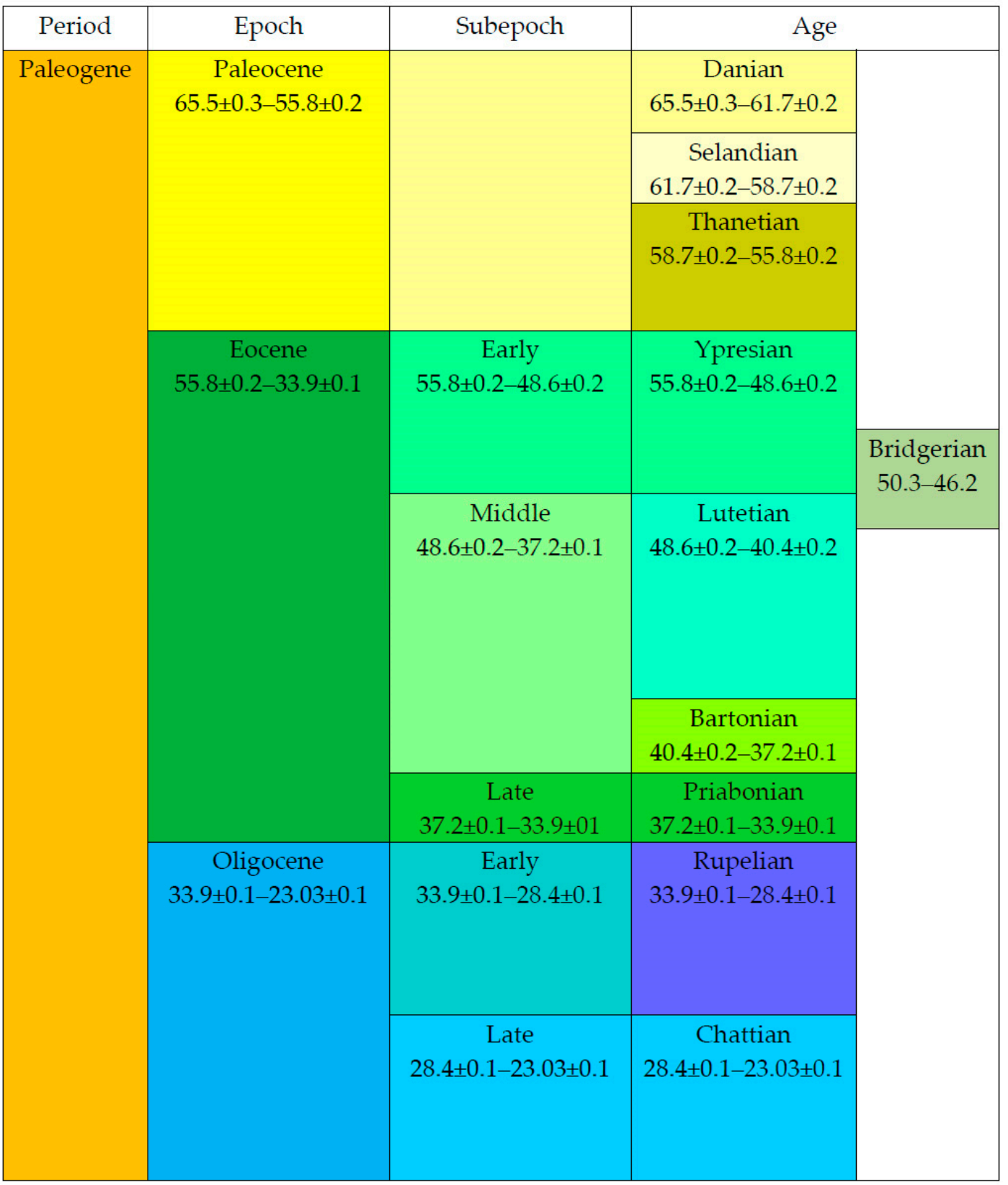

Figure 1. Geochronology of the Paleogene (Ma).

Curculionoidea have been found from 53 localities in 18 countries (Figure 2, Figure 3, Figure 4, Figure 5, Figure 6, Figure 9, Figure 12, Figure 14, Figure 20, Figure 21), spanning all ages of the Paleogene (Table 1). 


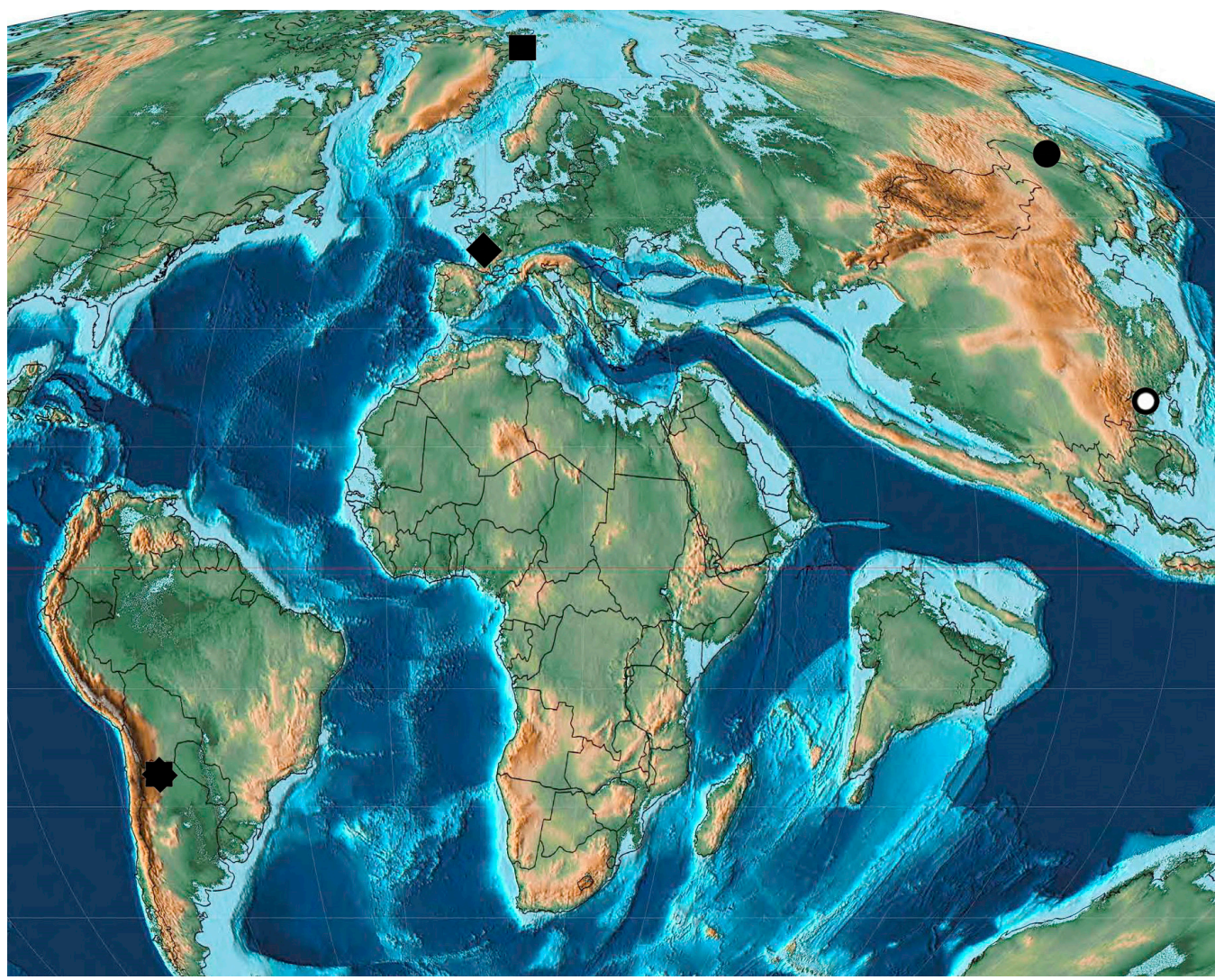

Figure 2. Paleocene Curculionoidea deposits: octagon—Sunchal; square—Starostin; rhombus-Menat; circle-Arkhara; ring-Mirs Bay.

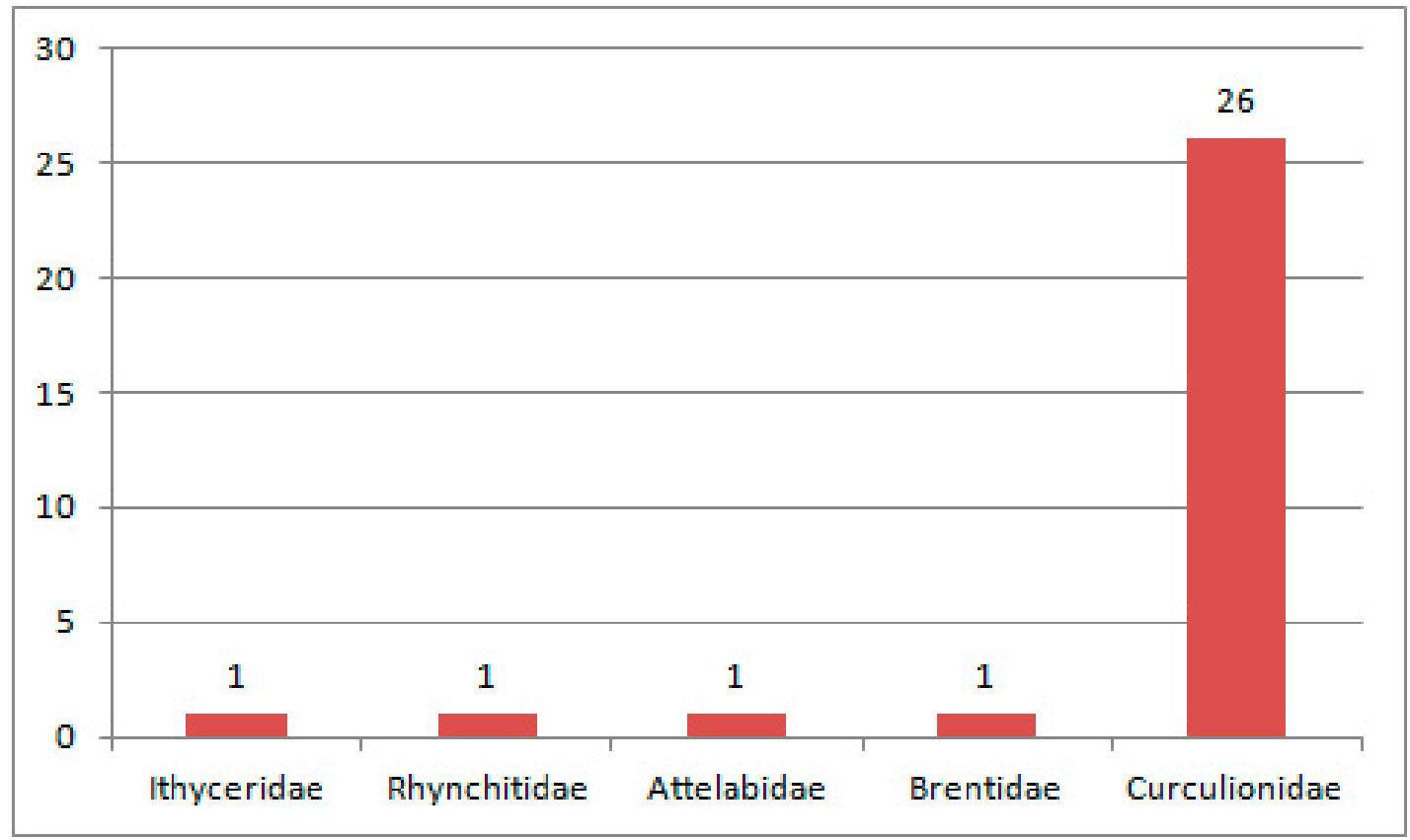

Figure 3. Composition of species of Curculionoidea in the Paleocene fauna. 


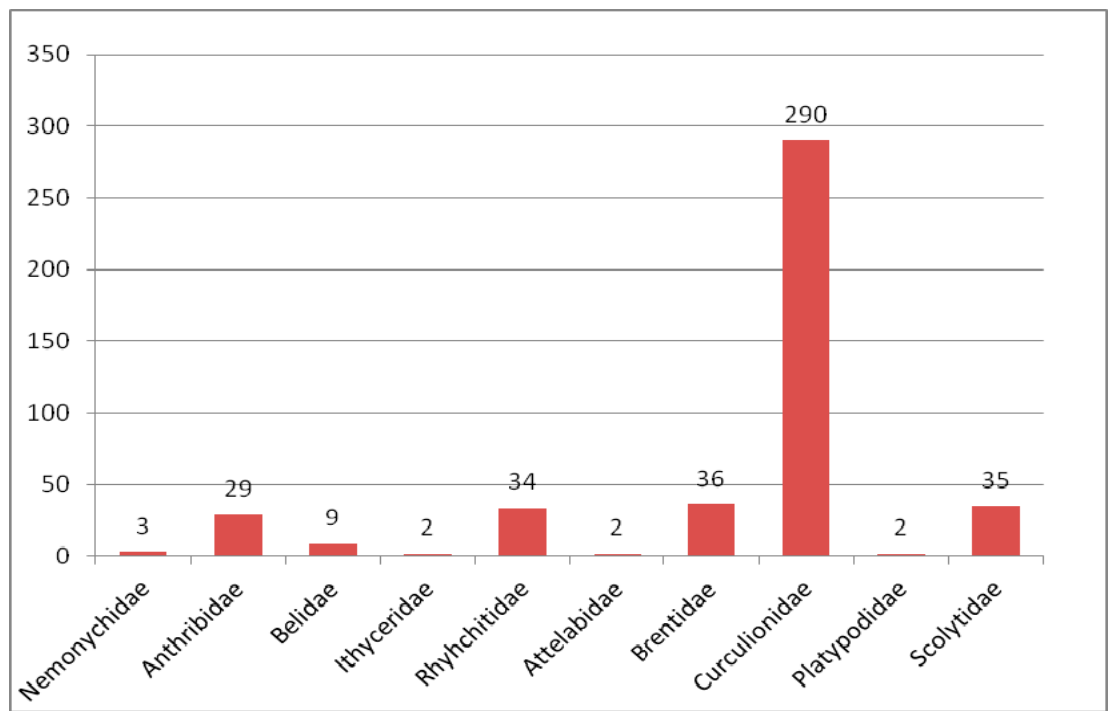

Figure 4. Composition of species of Curculionoidea in the Eocene fauna.

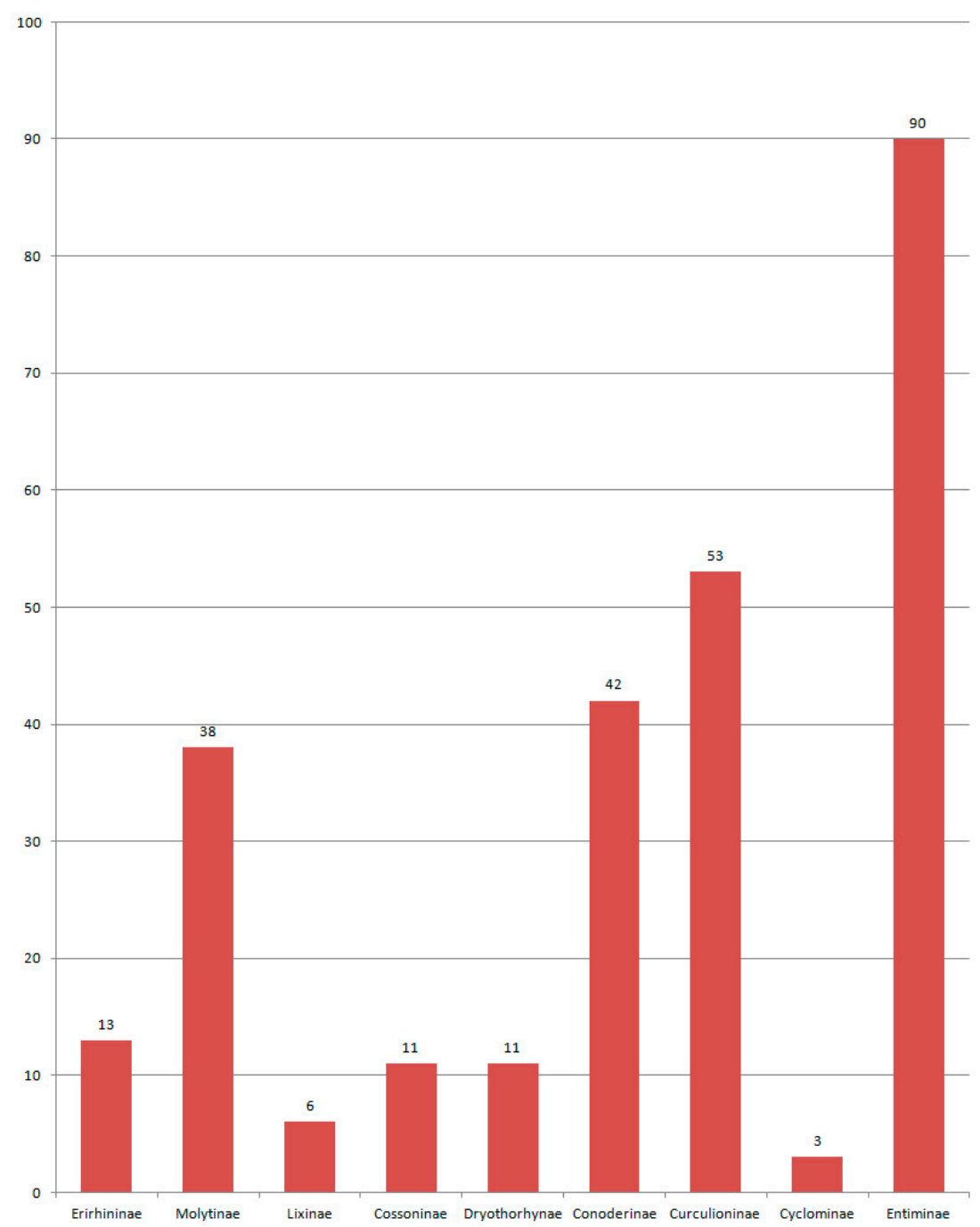

Figure 5. Composition of species of Curculionidae in the Eocene fauna. 


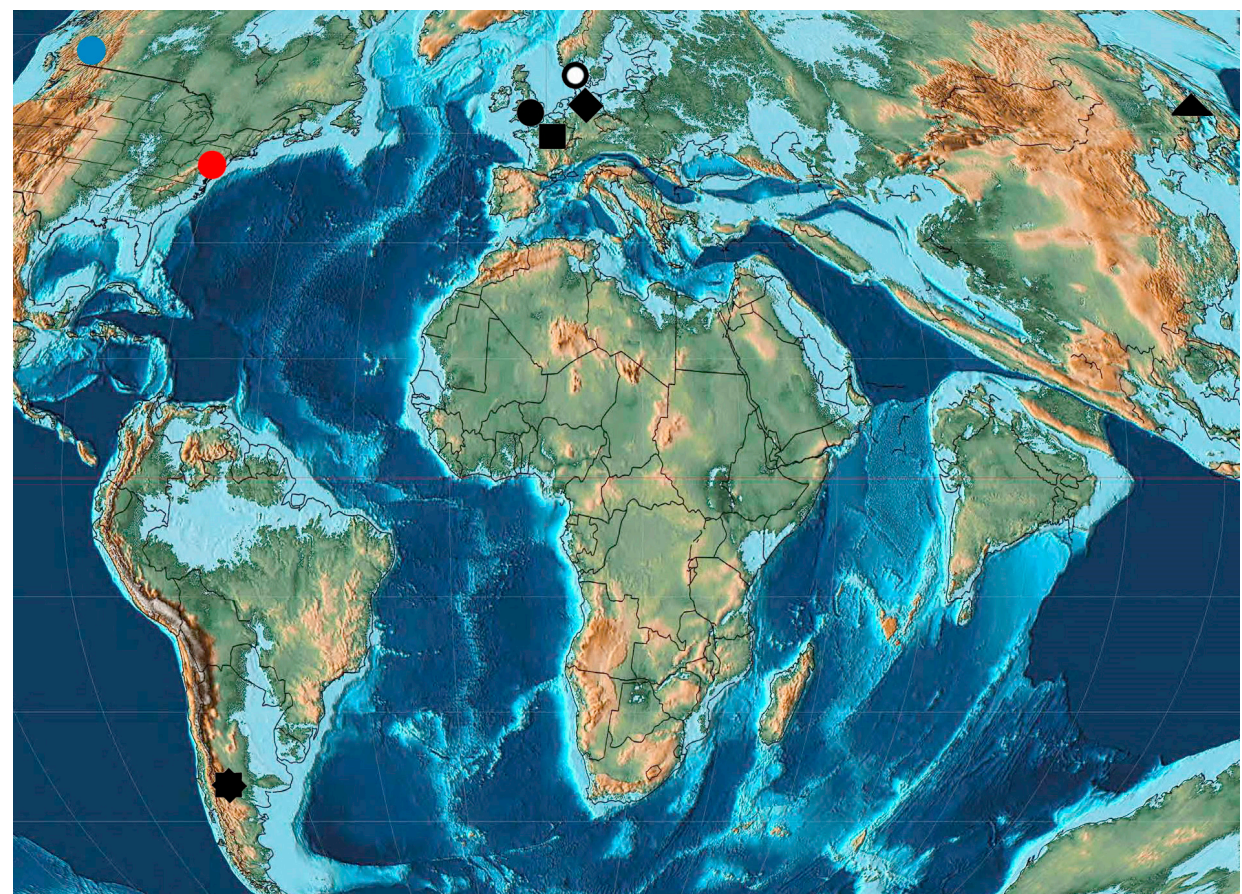

Figure 6. Early Eocene Curculionoidea deposits: square-Oise amber; circle-Peckham and London Clay; rhombus-Havighorst; ring-Mors; red circle-Republic; blue circle-Quilchena; triangle-Tadushi; octagon-Huitrera Formation.

Table 1. Localities with Curculionoidea from the Paleogene.

\begin{tabular}{|c|c|c|c|}
\hline Name & Abbreviation & Location & Age \\
\hline Arkhara & Arkh (P1d) & $\begin{array}{c}\text { Russia: Amurskaya Region, Arkharinskii District, } \\
\text { quarry at Arkhara Railway Station }\end{array}$ & $\begin{array}{l}\text { Lower Paleocene, } \\
\text { Danian, Cagayan }\end{array}$ \\
\hline Starostin & Star (P1d) & $\begin{array}{l}\text { Norway: Spitsbergen, Svalbard and Jan Mayen, } \\
\text { Firkanten Formation }\end{array}$ & $\begin{array}{l}\text { Lower Paleocene, } \\
\text { Danian, } 63 \pm 2 \mathrm{Ma}\end{array}$ \\
\hline Sunchal & Sunc (P1d) & $\begin{array}{l}\text { Argentina: northern Argentina, Jujuy Province, } \\
\text { La Mendieta, Maiz Gordo Formation }\end{array}$ & $\begin{array}{c}\text { Lower Paleocene, } \\
\text { Danian, } 66.0-55.8 \mathrm{Ma}\end{array}$ \\
\hline Menat & Mena (P1sl-t) & France: Puy-de-Dome & $\begin{array}{l}\text { Middle-Upper } \\
\text { Paleocene, } \\
\text { Selandian-Thanetian, } \\
61.0-59.0 \mathrm{Ma}\end{array}$ \\
\hline Mirs Bay & Mirs (P1) & $\begin{array}{l}\text { China: Hong Kong, Peng Chau Island, gray } \\
\text { sandy shales, Ping Chau Formation }\end{array}$ & Paleocene \\
\hline Mors & Mors (P2i) & Denmark, Fur Formation & $\begin{array}{c}\text { Lower Eocene, } \\
\text { Ypresian, } 54.0 \mathrm{Ma}\end{array}$ \\
\hline London Clay & LonC (P2i) & United Kingdom: England: Sussex, Bognor Regis & $\begin{array}{l}\text { Lower Eocene, } \\
\text { Ypresian, } \\
54.0-50.0 \mathrm{Ma}\end{array}$ \\
\hline Roan Mountain & RoaM (P2i-1) & United States: Colorado, Green River Formation & $\begin{array}{l}\text { Lower-Middle } \\
\text { Eocene, Bridgerian, } \\
53.5-48.5 \mathrm{Ma}\end{array}$ \\
\hline Green River & GreR (P2i-1) & $\begin{array}{c}\text { United States: Colorado, Wyoming, Utah States, } \\
3-4 \mathrm{~km} \text { west of railway crossing of Green River, } \\
\text { Green-River Formation }\end{array}$ & $\begin{array}{l}\text { Lower-Middle } \\
\text { Eocene, Bridgerian, } \\
53.5-48.5 \mathrm{Ma}\end{array}$ \\
\hline Oise amber & OisJ (P2i) & France: Paris basin, Creil, Oise & $\begin{array}{l}\text { Lowermost Eocene, } \\
\text { Ypresian, } 53.0 \mathrm{Ma}\end{array}$ \\
\hline
\end{tabular}


Table 1. Cont.

\begin{tabular}{|c|c|c|c|}
\hline Name & Abbreviation & Location & Age \\
\hline $\begin{array}{l}\text { Huitrera } \\
\text { Formation }\end{array}$ & & $\begin{array}{l}\text { Argentina: Arroyo Chacay, Río Negro, near } \\
\text { Estancia Don Hipólito, W, about } 60 \text { km east from } \\
\text { San Carlos de Bariloche, Huitrera Formation }\end{array}$ & $\begin{array}{l}\text { Lower Eocene, } \\
\text { Ypresian, } \\
54.24 \pm 0.45 \mathrm{Ma}\end{array}$ \\
\hline Peckham & Peck (P2i) & England: South London, Reding Beds & $\begin{array}{l}\text { Lower Eocene, } \\
\text { Ypresian }\end{array}$ \\
\hline Havighorst & Havi (P2i) & Germany: east of Hamburg, Schleswig Holstein & $\begin{array}{l}\text { Lower Eocene, } \\
\text { Ypresian }\end{array}$ \\
\hline Quilchena & & $\begin{array}{l}\text { Canada: British Columbia, } 3 \mathrm{~km} \text { south of Nicola } \\
\text { Lake, lacustrine shale }\end{array}$ & $\begin{array}{l}\text { Lower Eocene, } \\
\text { Ypresian, } \\
51.5 \pm 0.4 \mathrm{Ma}\end{array}$ \\
\hline Republic & Repu (P2i) & $\begin{array}{l}\text { United States: Northeast Washington State, } \\
\text { Klondike Mountain Formation }\end{array}$ & $\begin{array}{l}\text { Lower Eocene, } \\
\text { Ypresian, } \\
49.0-48.0 \mathrm{Ma}\end{array}$ \\
\hline Tadushi & Tadu (P2i) & $\begin{array}{l}\text { Russia: Russian Far East, Primorsky Krai, } \\
\text { Kavalerovsky District, Pestrushka River near } \\
\text { mouth and Ugol'nyi Creek, tributary of } \\
\text { Zerkal'nyi (Tadushi) River, near village of } \\
\text { Suvorovo, Tadushi Formation }\end{array}$ & $\begin{array}{l}\text { Lower Eocene, } \\
\text { Ypresian }\end{array}$ \\
\hline Messel & Mess (P21) & Germany: near Frankfurt, oil shales & $\begin{array}{l}\text { Middle Eocene, } \\
\text { Upper Ypresian } \\
\text { Lower Lutetian, } \\
48.27 \pm 0.22-47.0 \mathrm{Ma}\end{array}$ \\
\hline Corfe & Corf (P21) & $\begin{array}{l}\text { United Kingdom: England: Dorset, Corfe Clay, } \\
\text { Lower Bagshot Beds }\end{array}$ & $\begin{array}{l}\text { Middle Eocene, } \\
\text { Lutetian, }\end{array}$ \\
\hline Bournemouth & Bour (P21) & $\begin{array}{l}\text { United Kingdom: England, Dorset, Bagshot } \\
\text { Series }\end{array}$ & $\begin{array}{l}\text { Middle Eocene, } \\
\text { Lutetian, } \\
50.0-42.0 \mathrm{Ma}\end{array}$ \\
\hline Geiseltal & Geis (P2l) & Germany: near Halle & $\begin{array}{l}\text { Middle Eocene, } \\
\text { Lutetian, } \\
47.5-42.5 \mathrm{Ma}\end{array}$ \\
\hline Eckfelder Maar & Maar (P21) & Germany: Rheinland-Pfalz, Rhine Palatine & $\begin{array}{l}\text { Middle Eocene, } \\
\text { Lutetian, } 44.3 \mathrm{Ma}\end{array}$ \\
\hline $\begin{array}{l}\text { Baltic amber } \\
\text { (Kaliningrad } \\
\text { region) }\end{array}$ & BalJ (P2b) & $\begin{array}{c}\text { Russia: Kaliningrad Region, Baltic Sea coast and } \\
\text { Amber Jantarnyi quarry near Kaliningrad, } \\
\text { Prussian Formation }\end{array}$ & $\begin{array}{l}\text { Middle Eocene, } \\
\text { Bartonian, } \\
48.0-33.0 \mathrm{Ma}\end{array}$ \\
\hline Polish amber & PolJ (P2b) & $\begin{array}{l}\text { Poland: Gdansk city area, at the Wisla River } \\
\text { Estuary, Baltic amber, Prussian Formation; }\end{array}$ & $\begin{array}{l}\text { Middle Eocene, } \\
\text { Bartonian, } \\
48.0-33.0 \mathrm{Ma}\end{array}$ \\
\hline $\begin{array}{l}\text { Scandinavian } \\
\text { amber }\end{array}$ & ScanJ (P2b) & $\begin{array}{c}\text { Denmark, amber deposits on the Danish coast, } \\
\text { Baltic amber, Prussian Formation }\end{array}$ & $\begin{array}{l}\text { Middle Eocene, } \\
\text { Bartonian, } \\
48.0-33.0 \mathrm{Ma}\end{array}$ \\
\hline Rovno amber & $\operatorname{RovJ}(\mathbf{P} 2 b)$ & $\begin{array}{l}\text { Ukraine: Rovno Region, Klesov and Dubrovice } \\
\text { quarries }\end{array}$ & $\begin{array}{l}\text { Middle Eocene, } \\
\text { Bartonian, } \\
\text { 48.0-33.0 Ma }\end{array}$ \\
\hline $\begin{array}{l}\text { Bitterfeld } \\
\text { amber }\end{array}$ & & $\begin{array}{c}\text { Germany: Saxony-Anhalt, Goitzsche near } \\
\text { Bitterfeld }\end{array}$ & $\begin{array}{l}\text { Middle Eocene, } \\
\text { Bartonian, } \\
48.0-33.0 \mathrm{Ma} \\
\end{array}$ \\
\hline $\begin{array}{l}\text { Romanian } \\
\text { amber }\end{array}$ & & Romania: Alunisului Hill, Valea Sibiciului, Colti & $\begin{array}{l}\text { Middle Eocene, } \\
\text { Bartonian, } \\
48.0-33.0 \mathrm{Ma}\end{array}$ \\
\hline Kutschlin & Kuts (P2b) & Czech Republic, northwest Bohemia, near Bilina & $\begin{array}{l}\text { Middle Eocene, } \\
\text { Bartonian }\end{array}$ \\
\hline Celas & Cela (P2p) & $\begin{array}{c}\text { France: Gard Department, railway } \\
\text { Uze's-Saint-Julien-de-Casig-nac, Fumades, } \\
\text { Corents, Bassein Ales }\end{array}$ & $\begin{array}{l}\text { Upper Eocene, } \\
\text { Priabonian }\end{array}$ \\
\hline
\end{tabular}


Table 1. Cont.

\begin{tabular}{|c|c|c|c|}
\hline Name & Abbreviation & Location & Age \\
\hline Ales-Monteils & Ales (P2p) & France: Gard & $\begin{array}{l}\text { Upper Eocene, } \\
\text { Priabonian }\end{array}$ \\
\hline Florissant & Flor $(\mathbf{P} 2 p)$ & $\begin{array}{l}\text { United States: Colorado, Rocky Mountains near } \\
\text { Pike's Peak, Florissant Formation }\end{array}$ & $\begin{array}{c}\text { Latest Eocene, } \\
\text { Priabonian, } \\
34.07 \pm 0.10 \mathrm{Ma} \\
\end{array}$ \\
\hline Biamo & Biam (P2p) & $\begin{array}{l}\text { Russia: Russian Far East, Primorsky Region, } \\
\text { Pozharskii District, source of Barachek Creek, } \\
\text { right tributary of Bol'shaya Svetlovodnaya } \\
\text { (formerly Biamo) River }\end{array}$ & $\begin{array}{l}\text { Upper Eocene, } \\
\text { Priabonian }\end{array}$ \\
\hline Fonseca & Fons (P2p) & $\begin{array}{c}\text { Brazil: Minas Gerais State, near Fonseca } \\
\text { municipality, Whitish shales, under bituminous } \\
\text { shales, Fonseca Basin, Fonseca Formation }\end{array}$ & $\begin{array}{l}\text { Oligocene, } \\
\text { Priabonian }\end{array}$ \\
\hline $\begin{array}{l}\text { White River } \\
\text { Badlands }\end{array}$ & WhiR (P3r) & United States: South Dakota & $\begin{array}{l}\text { Lower Oligocene, } \\
\text { Rupelian, } \\
\text { 34.0-31.2 Ma }\end{array}$ \\
\hline Brunnstatt & Brun (P3r) & $\begin{array}{l}\text { France: Alsace, Haut-Rhine Dept., } 5 \mathrm{~km} \\
\text { southwest of Mulhouse }\end{array}$ & $\begin{array}{l}\text { Lower Oligocene, } \\
\text { Rupelian, 33.9 Ma } \\
\end{array}$ \\
\hline Corent & Core $(\mathbf{P} 3 \mathrm{r})$ & $\begin{array}{l}\text { France: South France, Puy-de-Dom Department, } \\
\text { Gergovia Plateau, south of Clermon-Ferran }\end{array}$ & $\begin{array}{c}\text { Lower Oligocene, } \\
\text { Rupelian } \\
\end{array}$ \\
\hline Cereste & & $\begin{array}{c}\text { France: Alpes-de-Haute Province, Basses Alp } \\
\text { Department }\end{array}$ & $\begin{array}{l}\text { Lower Oligocene, } \\
\text { Rupelian }\end{array}$ \\
\hline Seifhennersdorf & & Germany: Saxony, Oberlausitz & $\begin{array}{l}\text { Lower Oligocene, } \\
\text { Rupelian, } \\
\text { 30.5-30.2 Ma }\end{array}$ \\
\hline Ahuehuetes & & $\begin{array}{c}\text { Mexico: Puebla, } 4.5 \mathrm{~km} \text { NNE from Tepexi de } \\
\text { Rodrigues, Los Ahuehuetes, Coatzingo } \\
\text { Formation }\end{array}$ & $\begin{array}{l}\text { Lower Oligocene, } \\
\text { Rupelian }\end{array}$ \\
\hline Kundratice & & $\begin{array}{l}\text { Czech Republic: near Litomioice and } \\
\text { Seifhennersdorf }\end{array}$ & $\begin{array}{l}\text { Lower Oligocene, } \\
\text { Rupelian }\end{array}$ \\
\hline Sieblos & Sieb (P3) & Germany: Hessen, Rhon & Middle Oligocene \\
\hline Kleinkembs & Klei (P3) & $\begin{array}{l}\text { Germany: Baden-Wurtemberg, } 10 \mathrm{~km} \text { northwest } \\
\text { of Lorrach, Pays de Bade, Salt Formation }\end{array}$ & Middle Oligocene \\
\hline Gaube & Gaub (P3) & France: Puy-de-Dome, ravin de la Gaube & $\begin{array}{l}\text { Lower Oligocene, } \\
\text { Rupelian }\end{array}$ \\
\hline Puy-Saint-Jean & PuStJ (P3) & France: Puy-de-St. Jean & Middle Oligocene \\
\hline Luzice & Luzi (P3h) & Czech Republic: northwest Bohemia & $\begin{array}{l}\text { Upper Oligocene, } \\
\text { Chattian }\end{array}$ \\
\hline Enspel & & $\begin{array}{l}\text { Germany: Rheinland-Pfalz, Westerwald, } \\
\text { Bad Marienberg }\end{array}$ & $\begin{array}{l}\text { Upper Oligocene, } \\
\text { Chattian, } 24.79- \\
24.56 \mathrm{Ma}\end{array}$ \\
\hline Ashutas & & $\begin{array}{l}\text { Kazakhstan: East Kazakhstan Region, Kurshim } \\
\text { District, right bank of River Cherny Irtysh }\end{array}$ & $\begin{array}{l}\text { Upper Oligocene, } \\
\text { Chattian }\end{array}$ \\
\hline Perekishkyul' & & $\begin{array}{l}\text { Azerbaijan: middle course of Sumgait River, } \\
\text { village of Perekeshkul, Maikop Formation }\end{array}$ & $\begin{array}{l}\text { Upper Oligocene, } \\
\text { Chattian }\end{array}$ \\
\hline Rott & Rott (P3h) & $\begin{array}{c}\text { Germany: North Rhine-Westfalia, Siebengebirge, } \\
\text { near Bonn, Rott Formation }\end{array}$ & $\begin{array}{l}\text { Latest Oligocene, } \\
\text { Upper Chattian, } \\
24.0-23.0 \mathrm{Ma} \\
\end{array}$ \\
\hline Aix-en-Provance & Aix (P3h) & $\begin{array}{l}\text { France: Bouches-du-Rhane, Aix-en-Provence } \\
\text { Formation }\end{array}$ & $\begin{array}{l}\text { Latest Oligocene, } \\
\text { Upper Chattian }\end{array}$ \\
\hline Kap Dalton & KapD (P3) & Denmark: Greenland & Oligocene \\
\hline Kenderlyk II & & $\begin{array}{c}\text { Kazakhstan: East Kazakhstan Region, Zaisan } \\
\text { district, left bank of Kenderlyk River, } 6 \text { km from } \\
\text { village of Kenderlyk }\end{array}$ & Oligocene \\
\hline
\end{tabular}

Maps for Paleogene localities were from Scotese [1]. 
The ages of fossil deposits used are from the website Fossilworks-http://fossilworks.org and some publications [2-21]. Baltic amber from the Prussian Formation ranges from 33-48 million years (Lutetian-Bartonian) [22] and in this work the Bartonian age is accepted [23].

The systematics of the superfamily Curculionoidea is currently not stable [24-30]. Previously, it included the Mesozoic family Obrieniidae, the systematic position of which is assumed to be related to the Curculionoidea [31] or considered as a family within the Archostemata [32] or a separate superfamily within the Curculioniformia [30,33] and is not considered in this paper. The number of families, groups related to these families and their taxonomic status are not universally accepted [24-30,34-36]. Also the families Nemonychidae, Anthribidae, Belidae, Ithyceridae, Brentidae, Curculionidae, Platypodidae and Scolytidae not universally accepted. In this work the higher classification proposed by the author [29,30,37-42] is adopted. The subfamily Cimberidinae is considered part of the family Nemonychidae [26,29,37,38,43], in contrast to the opinion of Seunggwan et al. [44]. The families Anthribidae and Belidae are accepted in the traditional composition $[25,27,29,30,32,38,45]$. Ithyceridae and Caridae are often regarded as unrelated groups [25-28,32,36,44]; however, the author considers the family Ithyceridae (incl. Caridae and Ulyanidae) as a diverse, predominantly extinct group, including five fossil (Mongolocarinae, Baissorhynchinae, Montsecanomalinae, Ulyaninae, Slonikinae) and three recent subfamilies (Carinae, Chilecarinae, Ithycerinae) [29,30,38,46-51]. Rhynchitidae and Attelabidae are considered as independent families [30,38,52-61]. The family Brentidae consists of six subfamilies [38]. Nanophyinae and Apioninae, sometimes considered as separate families [26,62-64], are included in Brentidae [25,27,30,32,36,38]. The family Curculionidae includes Erirhininae according to Zherikhin and Egorov [65] and Legalov [30,41]. Scolytidae and Platypodidae are considered as separate families $[29,30,34,66]$.

Many Paleogene species were described in the 19th - first half of the 20th century in modern genera. The descriptions and illustrations of Many have been re-studied. If the generic affiliation was in doubt, then the genus has been placed in quotes. The results of these studies were presented by Legalov [30,47,50,67-71].

The studied fossil forms are deposited in-A. Bukejs's collection, Daugavpils, Latvia; A. Górski's collection, Bielsko-Biala, Poland; Borissiak Paleontological Institute of the Russian Academy of Sciences, Moscow, Russia; C. Gröhn's collection (Glinde, Germany) deposited in the Center of Natural History (formerly Geological-Paleontological Institute and Museum), Hamburg, Germany; Center of Natural History (formerly Geological-Paleontological Institute and Museum), Hamburg, Germany; Centre de Conservation du musée des confluences, Lyon, France; Earth Institute, Warsaw, Poland; F. Kernegger's collection, Hamburg, Germany, deposited in the Forschungsinstitut Senckenberg, Frankfurt am Main, Germany; Friedhelm Eichmann, Hannover, Germany; Górnośląskie Muzeum Przyrodnicze w Bytomiu, Poland; Kaliningrad Regional Amber Museum, Kaliningrad, Russia; Legalov's fossil insects collection Maintained at Institute of Systematics and Ecology of Animals of the Siberian Branch of the Russian Academy of Science, Novosibirsk, Russia; Muséum national d'histoire naturelle, Paris, France; Museum of Amber Inclusions, University of Gdańsk, Poland; Museum of the World Ocean, Kaliningrad, Russia; Naturhistorisches Museum Mainz, Landessammlung für Naturkunde Rheinland-Pfalz; Poinar amber collection Maintained at Oregon State University, Corvallis, OR, USA; Schmalhausen Institute of Zoology of the National Academy of Sciences of Ukraine, Kiev, Ukraine; V. Alekseev's collection, Kaliningrad, Russia; V. Gusakov's collection, Russia, Moscow; Zoological Museum, University of Copenhagen, Denmark.

The specimens were studied using a stereomicroscope Zeiss Stemi 2000-C in the Institute of Systematic and Ecology of Animals (Novosibirsk, a Leica M165C binocular microscope, with Leica DFG 425, MBS 10, MBS 12 in the Paleontological Institute (Moscow), a Leica MZ 16.0 stereomicroscope with a DFC290 camera in the Zoological Institute (St. Petersburg) and an Olympus SCX9 stereomicroscope with an Olympus camera and a Nikon SMZ1500 with Microscope Eyepiece Camera 9.0MP Aptina Color CMOS MU900 in the Muséum National d'Histoire Naturelle (Paris). 
The present work is registered in ZooBank (www.zoobank.org) under LSID urn-lsid:zoobank.org:pub:125CCA9E-7288-4C12-88C9-BDEBDC300A9C.

\section{Results}

Four species of the family Nemonychidae, 33 species of the family Anthribidae, three species of the family Ithyceridae, nine species of the family Belidae, 41 species of the family Rhynchitidae, three species of the family Attelabidae, 47 species of the family Brentidae, 384 species of the family Curculionidae, two species of the family Platypodidae and 37 species of the family Scolytidae were described from the Paleogene (Table 2).

Table 2. List of species of Curculionoidea from Paleogene [8,29,30,47,50,67,69-175].

\begin{tabular}{|c|c|c|c|c|}
\hline No. & Taxon & Locality & Age & Sources \\
\hline \multirow{3}{*}{\multicolumn{5}{|c|}{$\begin{array}{c}\text { Nemonychidae Bedel, } 1882 \\
\text { Cretonemonychinae Gratshev et Legalov, } 2009 \\
\text { Eocaenonemonychini Legalov, } 2013\end{array}$}} \\
\hline & & & & \\
\hline & & & & \\
\hline 1 & Eocaenonemonyx kuscheli Legalov, 2013 & GreR & $\mathrm{P} 2 \mathrm{i}-1$ & 72 \\
\hline 2 & "Sitones" grandaevus Scudder, 1876 & GreR & P2i-1 & 73 \\
\hline 3 & "Eugnamptus" decemsatus Scudder, 1878 & KapD & P3 & 74 \\
\hline \multirow{2}{*}{\multicolumn{5}{|c|}{$\begin{array}{c}\text { Cimberidinae Gozis, } 1882 \\
\text { Kuschelomacerini Riedel, } 2010\end{array}$}} \\
\hline & & & & \\
\hline 4 & Kuschelomacer kerneggeri Riedel, 2010 & BalJ & $\mathrm{P} 2 \mathrm{~b}$ & 75 \\
\hline \multirow{3}{*}{\multicolumn{5}{|c|}{$\begin{array}{l}\text { Anthribidae Billberg, } 1820 \\
\text { Anthribinae Billberg, } 1820 \\
\text { Cratoparini LeConte, } 1876\end{array}$}} \\
\hline & & & & \\
\hline & & & & \\
\hline 5 & "Euparius" elusus (Scudder, 1878) & GreR & P2i-1 & 74 \\
\hline 6 & "E." repertus (Scudder, 1878) & Flor & $\mathrm{P} 2 \mathrm{p}$ & 74 \\
\hline 7 & "E." adumbratus (Wickham, 1911) & Flor & $\mathrm{P} 2 \mathrm{p}$ & 76 \\
\hline 8 & "E." arcessitus (Scudder, 1893) & Flor & $\mathrm{P} 2 \mathrm{p}$ & 77 \\
\hline \multicolumn{5}{|c|}{ Anthribini Billberg, 1820} \\
\hline 9 & "Ormiscus" partitus (Scudder, 1890) & GreR & P2i-1 & 78 \\
\hline 10 & “Trigonorhinus" pristinus (Scudder, 1876) & GreR & P2i-1 & 73 \\
\hline 11 & "T." sordidus (Scudder, 1893) & Flor & $\mathrm{P} 2 \mathrm{p}$ & 77 \\
\hline \multicolumn{5}{|c|}{ Ecelonerini Lacordaire, 1865} \\
\hline 12 & Pseudochirotenon eocaenicus Legalov, 2018 & GreR & P2i-1 & 69 \\
\hline 13 & Saperdirhynchus prisctitillator Scudder, 1893 & Flor & $\mathrm{P} 2 \mathrm{p}$ & 77 \\
\hline \multicolumn{5}{|c|}{ Mecocerini Lacordaire, 1865} \\
\hline 14 & Pseudoecocerus aleksevi Legalov, 2020 & BalJ & $\mathrm{P} 2 \mathrm{~b}$ & 71 \\
\hline \multicolumn{5}{|c|}{ Tropiderini Lacordaire, 1865} \\
\hline 15 & "Tropideres" remotus Scudder, 1893 & GreR & P2i-1 & 77 \\
\hline 16 & "T." vastats Scudder, 1893 & Flor & $\mathrm{P} 2 \mathrm{p}$ & 77 \\
\hline \multicolumn{5}{|c|}{ Allandrini Pierce, 1930} \\
\hline 17 & Pseudomecorhis orlovi Zherikhin, 1971 & BalJ & $\mathrm{P} 2 \mathrm{~b}$ & 79 \\
\hline 18 & P. simulator Voss, 1953 & BalJ & $\mathrm{P} 2 \mathrm{~b}$ & 80 \\
\hline 19 & Allandroides vossi Legalov, 2015 & BalJ & $\mathrm{P} 2 \mathrm{~b}$ & 30 \\
\hline \multicolumn{5}{|c|}{ Oiserhinini Legalov, Kirejtshuk et Nel, 2019} \\
\hline 20 & Oiserhinus insolitus Legalov, Kirejtshuk et Nel, 2019 & OisJ & $\mathrm{P} 2 \mathrm{i}$ & 81 \\
\hline \multicolumn{5}{|c|}{ Zygaenodini Lacordaire, 1865} \\
\hline 21 & Glaesotropis (Pseudoglaesotropis) balticus Legalov, 2020 & BalJ & $\mathrm{P} 2 \mathrm{~b}$ & 71 \\
\hline
\end{tabular}


Table 2. Cont.

\begin{tabular}{|c|c|c|c|c|}
\hline No. & Taxon & Locality & Age & Sources \\
\hline 22 & G. (P.) martynovi (Legalov, 2012) & BalJ & $\mathrm{P} 2 \mathrm{~b}$ & 82 \\
\hline 23 & G. (Glaesotropis) diadiasashai Gratshev et Perkovsky, 2008 & RovJ & $\mathrm{P} 2 \mathrm{~b}$ & 83 \\
\hline 24 & G. (G.) gusakovi Legalov, 2015 & BalJ & $\mathrm{P} 2 \mathrm{~b}$ & 30 \\
\hline 25 & G. (G.) minor Gratshev et Zherikhin, 1995 & BalJ & $\mathrm{P} 2 \mathrm{~b}$ & 84 \\
\hline 26 & G. (G.) succiniferus Legalov, 2015 & Balj & $\mathrm{P} 2 \mathrm{~b}$ & 30 \\
\hline 27 & G. (G.) weitschati Gratshev et Zherikhin, 1995 & BalJ & $\mathrm{P} 2 \mathrm{~b}$ & 84 \\
\hline 28 & G. (Electranthribus) alleni Legalov, 2015 & BalJ & $\mathrm{P} 2 \mathrm{~b}$ & 30 \\
\hline 29 & G. (E.) gratshevi Legalov, 2015 & BalJ & $\mathrm{P} 2 \mathrm{~b}$ & 30 \\
\hline 30 & G. (E.) zherikhini (Legalov, 2013) & BalJ & $\mathrm{P} 2 \mathrm{~b}$ & 47 \\
\hline \multicolumn{5}{|c|}{ Tribe incertae sedis } \\
\hline 31 & Stiraderes conradi Scudder, 1893 & Flor & $\mathrm{P} 2 \mathrm{p}$ & 77 \\
\hline \multicolumn{5}{|c|}{$\begin{array}{l}\text { Choraginae W. Kirby, } 1819 \\
\text { Choragini W. Kirby, } 1819\end{array}$} \\
\hline 32 & "Choragus" fictilis Scudder, 1890 & GreR & P2i-1 & 78 \\
\hline 33 & "Ch." tertiarius Heyden et Heyden, 1866 & Rott & P3h & 85 \\
\hline \multicolumn{5}{|c|}{ Valenfriesiini Alonso-Zarazaga et Lyal, 1999} \\
\hline 34 & $\begin{array}{l}\text { Eduardoxenus unicus Legalov, Nazarenko et Perkovsky, } \\
2018\end{array}$ & RovJ & $\mathrm{P} 2 \mathrm{~b}$ & 86 \\
\hline \multicolumn{5}{|c|}{ Urodontinae C.G Thomson, 1859} \\
\hline 35 & "Bruchela" cincta (Foerster, 1891) & Brun & $\mathrm{P} 3 \mathrm{r}$ & 87 \\
\hline 36 & “B." priscus (C. Heyden, 1862) & Rott & P3h & 88 \\
\hline 37 & "B." multipunctata (Schlechtendal, 1894) & Rott & P3h & 89 \\
\hline \multicolumn{5}{|c|}{$\begin{array}{c}\text { Ithyceridae Schoenherr, } 1823 \\
\text { Chilecarinae Legalov, } 2009 \\
\text { Chilecarini Legalov, } 2009\end{array}$} \\
\hline 38 & Petropsis rostratus Legalov, Kirejtshuk et Nel, 2017 & Mena & P1sl-t & 50 \\
\hline \multicolumn{5}{|c|}{ Ithycerinae Schoenherr, 1823} \\
\hline 39 & Eoceneithycerus carpenteri Legalov, 2013 & Repu & P2i & 47 \\
\hline 40 & Ithyceroides klondikensis Legalov, 2015 & Repu & $\mathrm{P} 2 \mathrm{i}$ & 30 \\
\hline \multicolumn{5}{|c|}{$\begin{array}{c}\text { Belidae Schoenherr, } 1826 \\
\text { Oxycoryninae Schoenherr, } 1840 \\
\text { Oxycraspedini Marvaldi et Oberprieler, } 2006\end{array}$} \\
\hline 41 & Oxycraspedus poinari Legalov, 2016 & BalJ & $\mathrm{P} 2 \mathrm{~b}$ & 90 \\
\hline \multicolumn{5}{|c|}{$\begin{array}{l}\text { Metrioxenini Voss, } 1953 \\
\text { Metrioxenina Voss, } 1953\end{array}$} \\
\hline 42 & Archimetrioxena electrica Voss, 1953 & BalJ & $\mathrm{P} 2 \mathrm{~b}$ & 80 \\
\hline 43 & A. zherikhini (Legalov, 2012) & BalJ & $\mathrm{P} 2 \mathrm{~b}$ & 82 \\
\hline \multicolumn{5}{|c|}{ Zherichinixenina Legalov, 2009} \\
\hline 44 & Paltorhynchus bisculcatus Scudder, 1893 & RoaM & P2i-1 & 77 \\
\hline 45 & P. narwhal Scudder, 1893 & Flor & $\mathrm{P} 2 \mathrm{p}$ & 77 \\
\hline 46 & Succinometrioxena attenuata Legalov et Poinar, 2020 & BalJ & $\mathrm{P} 2 \mathrm{~b}$ & 91 \\
\hline 47 & S. bachofeni Legalov, 2013 & BalJ & $\mathrm{P} 2 \mathrm{~b}$ & 47 \\
\hline 48 & S. poinari Legalov, 2012 & BalJ & $\mathrm{P} 2 \mathrm{~b}$ & 92 \\
\hline \multicolumn{5}{|c|}{$\begin{array}{l}\text { Allocorynitae Sharp, } 1890 \\
\text { Palaeorhopalotriini Legalov, } 2013\end{array}$} \\
\hline 49 & Palaeorhopalotria neli Legalov, 2013 & Ales & $\mathrm{P} 2 \mathrm{p}$ & 47 \\
\hline & $\begin{array}{l}\text { Rhynchitidae Gistel, } 1856 \\
\text { Sayrevilleinae Legalov, } 2003 \\
\text { Sanyrevilleini Legalov, } 2003\end{array}$ & & & \\
\hline
\end{tabular}


Table 2. Cont.

\begin{tabular}{|c|c|c|c|c|}
\hline No. & Taxon & Locality & Age & Sources \\
\hline 50 & Baltocar convexus Legalov, 2015 & BalJ & $\mathrm{P} 2 \mathrm{~b}$ & 30 \\
\hline 51 & B. groehni Riedel, 2012 & BalJ & $\mathrm{P} 2 \mathrm{~b}$ & 93 \\
\hline 52 & B. hoffeinsorum Riedel, 2012 & BalJ & $\mathrm{P} 2 \mathrm{~b}$ & 93 \\
\hline 53 & B. subnudus Riedel, 2012 & BalJ & $\mathrm{P} 2 \mathrm{~b}$ & 93 \\
\hline 54 & B. succinicus (Voss, 1953) & BalJ & $\mathrm{P} 2 \mathrm{~b}$ & 80 \\
\hline \multicolumn{5}{|c|}{ Vossicartini Legalov, 2003} \\
\hline 55 & Germanocartus heydeni (Schlechtendal, 1894) & Rott & P3h & 89 \\
\hline \multirow{3}{*}{\multicolumn{5}{|c|}{$\begin{array}{l}\text { Rhynchitinae Gistel, } 1856 \\
\text { Auletini Desbrochers des Loges, } 1908 \\
\text { Auletina Desbrochers des Loges, } 1908\end{array}$}} \\
\hline & & & & \\
\hline & & & & \\
\hline 56 & Electrauletes unicus Legalov, 2015 & BalJ & $\mathrm{P} 2 \mathrm{~b}$ & 30 \\
\hline \multicolumn{5}{|c|}{ Pseudauletina Voss, 1933} \\
\hline 57 & Eoropseudauletes plucinskii Kania et Legalov, 2019 & BalJ & $\mathrm{P} 2 \mathrm{~b}$ & 94 \\
\hline \multicolumn{5}{|c|}{ Pseudomesauletina Legalov, 2003} \\
\hline 58 & Pseudomesauletes groehni Bukejs et Legalov, 2019 & RovJ & $\mathrm{P} 2 \mathrm{~b}$ & 95 \\
\hline 59 & P. culex (Scudder, 1893) & Flor & $\mathrm{P} 2 \mathrm{p}$ & 77 \\
\hline 60 & P. ibis (Wickham, 1912) & Flor & $\mathrm{P} 2 \mathrm{p}$ & 96 \\
\hline 61 & P. obliquus (Wickham, 1913) & Flor & $\mathrm{P} 2 \mathrm{p}$ & 97 \\
\hline 62 & P. striaticeps (Wickham, 1911) & Flor & $\mathrm{P} 2 \mathrm{p}$ & 76 \\
\hline \multicolumn{5}{|c|}{ Subtribe incertae sedis } \\
\hline 63 & “Teretrum” quiescitum Scudder, 1893 & GreR & P2i-1 & 77 \\
\hline 64 & T. primulum Scudder, 1893 & Flor & $\mathrm{P} 2 \mathrm{p}$ & 77 \\
\hline 65 & Docirhynchus terebrans Scudder, 1893 & Flor & $\mathrm{P} 2 \mathrm{p}$ & 77 \\
\hline 66 & "Trypanorhynchus" depratus Scudder, 1893 & Flor & $\mathrm{P} 2 \mathrm{p}$ & 77 \\
\hline 67 & "Paltorhynchus" rectirostris Scudder, 1893 & Flor & $\mathrm{P} 2 \mathrm{p}$ & 77 \\
\hline 68 & “Trypanorhynchus" sedatus Scudder, 1893 & Flor & $\mathrm{P} 2 \mathrm{p}$ & 77 \\
\hline \multirow{2}{*}{\multicolumn{5}{|c|}{$\begin{array}{l}\text { Rhynchitini Gistel, } 1856 \\
\text { Temnocerina Legalov, } 2003\end{array}$}} \\
\hline & & & & \\
\hline 69 & Eocenorhynchites vossi Legalov, 2012 & BalJ & $\mathrm{P} 2 \mathrm{~b}$ & 74 \\
\hline \multicolumn{5}{|c|}{ Perrhynchitina Legalov, 2003} \\
\hline 70 & Succinorhynchites alberti Legalov, 2013 & BalJ & $\mathrm{P} 2 \mathrm{~b}$ & 47 \\
\hline 71 & Tatianaerhynchites goergesi (Zherikhin, 1992) & Rott & P3h & 98 \\
\hline \multicolumn{5}{|c|}{ Rhynchitina Gistel, 1856} \\
\hline 72 & Cartorhynchites struvei Zherikhin, 1992 & Rott & P3h & 98 \\
\hline 73 & Opacoinvolvulus rottensis (Zherikhin, 1992) & Rott & P3h & 98 \\
\hline 74 & O. zherichini Legalov, 2003 & Rott & P3h & 55 \\
\hline \multicolumn{5}{|c|}{ Subtribe incertae sedis } \\
\hline 75 & "Rhysosternum" punctatolineatum Piton, 1940 & Mena & P1sl-t & 99 \\
\hline 76 & Isothea alleni Scudder, 1893 & Flor & $\mathrm{P} 2 \mathrm{p}$ & 77 \\
\hline & =Trypanorhynchus corruptivus Scudder, 1893 & & & 77 \\
\hline 77 & Prodeporaus curiosum (Scudder, 1893) & Flor & $\mathrm{P} 2 \mathrm{p}$ & 77 \\
\hline 78 & P. exanimale (Scudder, 1893) & Flor & $\mathrm{P} 2 \mathrm{p}$ & 77 \\
\hline 79 & P. exilis (Wickham, 1913) & Flor & $\mathrm{P} 2 \mathrm{p}$ & 97 \\
\hline 80 & P. minutissimus (Wickham, 1913) & Flor & $\mathrm{P} 2 \mathrm{p}$ & 97 \\
\hline 81 & P. smithii (Scudder, 1893) & Flor & $\mathrm{P} 2 \mathrm{p}$ & 77 \\
\hline 82 & "Prodeporaides" laminarum (Wickham, 1916) & Flor & $\mathrm{P} 2 \mathrm{p}$ & 100 \\
\hline 83 & "P." subterraneus (Scudder, 1893) & Flor & $\mathrm{P} 2 \mathrm{p}$ & 77 \\
\hline 84 & "P." vulcan (Wickham, 1916) & Flor & $\mathrm{P} 2 \mathrm{p}$ & 100 \\
\hline 85 & P. wymani (Scudder, 1893) & Flor & $\mathrm{P} 2 \mathrm{p}$ & 77 \\
\hline 86 & "Masteutes" saxifer Scudder, 1893 & Flor & $\mathrm{P} 2 \mathrm{p}$ & 77 \\
\hline 87 & "Rhynchites" hageni Heyden et Heyden, 1866 & Rott & P3h & 85 \\
\hline
\end{tabular}


Table 2. Cont.

\begin{tabular}{|c|c|c|c|c|}
\hline No. & Taxon & Locality & Age & Sources \\
\hline 110 & Apionion evestigatum (Scudder, 1893) & RoaM & P2i-1 & 77 \\
\hline 111 & Toxorhynchus europeoeocenicus Bukejs et Legalov, 2020 & RovJ & $\mathrm{P} 2 \mathrm{~b}$ & 108 \\
\hline 112 & T. michalskii Legalov, in print. & PolJ & $\mathrm{P} 2 \mathrm{~b}$ & 109 \\
\hline 113 & "T." arctus (Scudder, 1893) & Flor & $\mathrm{P} 2 \mathrm{p}$ & 77 \\
\hline 114 & "T." confectum (Scudder, 1893) & Flor & P2p & 77 \\
\hline 115 & “T." corruptus (Scudder, 1893) & Flor & $\mathrm{P} 2 \mathrm{p}$ & 77 \\
\hline 116 & T. florissantensis (Wickham, 1916) & Flor & $\mathrm{P} 2 \mathrm{p}$ & 100 \\
\hline 117 & T. minusculus (Scudder, 1893 & Flor & $\mathrm{P} 2 \mathrm{p}$ & 77 \\
\hline 118 & T. oculatus (Scudder, 1893 & Flor & $\mathrm{P} 2 \mathrm{p}$ & 77 \\
\hline 119 & “T." pumilum (Scudder, 1893) & Flor & P2p & 77 \\
\hline 120 & "T." refrenatum (Scudder, 1893) & Flor & P2p & 77 \\
\hline 121 & “T." reventus (Scudder, 1893) & Flor & $\mathrm{P} 2 \mathrm{p}$ & 77 \\
\hline 122 & T. scudderianum (Wickham, 1916) & Flor & $\mathrm{P} 2 \mathrm{p}$ & 100 \\
\hline \multicolumn{5}{|c|}{ Tribe incertae sedis } \\
\hline 123 & "Apion" cockerelli Wickham, 1911 & Flor & P2p & 76 \\
\hline 124 & "A." obtusus (Scudder, 1893) & Flor & $\mathrm{P} 2 \mathrm{p}$ & 77 \\
\hline 125 & “A." levirostre Foerster, 1891 & Brun & $\mathrm{P} 3 \mathrm{r}$ & 87 \\
\hline 126 & "A." parvum Foerster, 1891 & Brun & $\mathrm{P} 3 \mathrm{r}$ & 87 \\
\hline 127 & "A." sulcatum Foerster, 1891 & Brun & $\mathrm{P} 3 \mathrm{r}$ & 87 \\
\hline 128 & "Rhynchites" orcinus Heyden et Heyden, 1866 & Rott & P3h & 85 \\
\hline 129 & "Apion" primordiale Heyden et Heyden, 1866 & Rott & P3h & 85 \\
\hline 130 & "A." profundum Schlechtendal, 1894 & Rott & P3h & 89 \\
\hline 131 & "Coeliodes" primigenius Oustalet, 1874 & Aix & P3h & 110 \\
\hline \multicolumn{5}{|c|}{$\begin{array}{c}\text { Nanophyinae Gistel, } 1856 \\
\text { Nanophyini Gistel, } 1856\end{array}$} \\
\hline 132 & Palaeonanophyes zherikhini Legalov, 2015 & GreR & P2i-1 & 111 \\
\hline 133 & Baltonanophyes crassirostre Legalov, 2018 & PolJ & $\mathrm{P} 2 \mathrm{~b}$ & 104 \\
\hline 134 & Zherikhiniellus rarus Legalov, in print. & BalJ & $\mathrm{P} 2 \mathrm{~b}$ & 109 \\
\hline 135 & "Nanophyes" japetus Heyden et Heyden, 1866 & Rott & P3h & 85 \\
\hline \multicolumn{5}{|c|}{ Corimaliini Alonso-Zarazaga, 1989} \\
\hline 136 & "Corimalia" cycloptera Theobald, 1937 & Cela & P2p & 112 \\
\hline \multirow{3}{*}{\multicolumn{5}{|c|}{$\begin{array}{c}\text { Brentinae Billberg, } 1820 \\
\text { Trachelizini Lacordaire, } 1865 \\
\text { Stereodermina Sharp, } 1895\end{array}$}} \\
\hline & & & & \\
\hline & & & & \\
\hline 137 & $\begin{array}{c}\text { Cerobates (Cerobates) eocenicus Legalov et Wappler, in } \\
\text { print. }\end{array}$ & Maar & P21 & 113 \\
\hline \multicolumn{5}{|c|}{ Trachelizina Lacordaire, 1865} \\
\hline 138 & Eckfelderolispa petrefacta Legalov et Wappler, in print. & Maar & P21 & 113 \\
\hline 139 & E. perita Legalov et Wappler, in print. & Maar & $\mathrm{P} 21$ & 113 \\
\hline 140 & E. manderschieta Legalov et Wappler, in print. & Maar & P21 & 113 \\
\hline \multirow{4}{*}{\multicolumn{5}{|c|}{$\begin{array}{c}\text { Curculionidae Latreille, } 1802 \\
\text { Erirhininae Schoenherr, } 1825 \\
\text { Erirhinini Schoenherr, } 1825 \\
\text { Erirhinina Schoenherr, } 1825\end{array}$}} \\
\hline & & & & \\
\hline & & & & \\
\hline & & & & \\
\hline 141 & Erirhinites bognorensis Britton, 1960 & LonC & P2i & 114 \\
\hline 142 & "Procas" vinculatus Scudder 1893 & $\begin{array}{l}\text { RoaM, } \\
\text { WhiR }\end{array}$ & $\begin{array}{c}\text { P2i-l, } \\
\text { P3r }\end{array}$ & 77 \\
\hline 143 & "P." verberatus Scudder, 1893 & Flor & $\mathrm{P} 2 \mathrm{p}$ & 77 \\
\hline 144 & "Erycus" brevicollis Scudder, 1893 & Flor & $\mathrm{P} 2 \mathrm{p}$ & 77 \\
\hline
\end{tabular}


Table 2. Cont.

\begin{tabular}{|c|c|c|c|c|}
\hline No. & Taxon & Locality & Age & Sources \\
\hline \multicolumn{5}{|c|}{ Dorytomini Bedel, 1886} \\
\hline 145 & Dorytomus bukejsi Legalov, 2020 & BalJ & $\mathrm{P} 2 \mathrm{~b}$ & 71 \\
\hline 146 & D. electrinus Legalov, 2016 & BalJ & $\mathrm{P} 2 \mathrm{~b}$ & 90 \\
\hline 147 & D. groehni Bukejs et Legalov, 2019 & BalJ & $\mathrm{P} 2 \mathrm{~b}$ & 116 \\
\hline 148 & D. korotyaevi Legalov, 2020 & BalJ & $\mathrm{P} 2 \mathrm{~b}$ & 71 \\
\hline 149 & D. nudus Legalov, 2016 & BalJ & $\mathrm{P} 2 \mathrm{~b}$ & 115 \\
\hline 150 & D. vlaskini Legalov, Nazarenko et Perkovsky, 2019 & RovJ & $\mathrm{P} 2 \mathrm{~b}$ & 117 \\
\hline 151 & “D." coercitus Scudder, 1893 & Flor & $\mathrm{P} 2 \mathrm{p}$ & 77 \\
\hline 152 & “D.” vulcanicus Wickham, 1912 & Flor & $\mathrm{P} 2 \mathrm{p}$ & 101 \\
\hline 153 & “D.” williamsi Scudder, 1893 & Flor & $\mathrm{P} 2 \mathrm{p}$ & 77 \\
\hline \multicolumn{5}{|c|}{ Bagoini C.G. Thomson, 1859} \\
\hline 154 & “Bagous" palintonus Foerster, 1891 & Brun & $\mathrm{P} 3 \mathrm{r}$ & 87 \\
\hline \multirow{3}{*}{\multicolumn{5}{|c|}{$\begin{array}{c}\text { Molytinae Schoenherr, } 1823 \\
\text { Molytini Schoenherr, } 1823 \\
\text { Hylobiina W. Kirby, } 1837\end{array}$}} \\
\hline & & & & \\
\hline & & & & \\
\hline 155 & Archaralites zherichini Legalov, 2010 & Arkh & P1d & 118 \\
\hline 156 & Furhylobius troesteri Legalov, 2015 & Mors & $\mathrm{P} 2 \mathrm{i}$ & 30 \\
\hline 157 & Archaeoheilus packardii (Scudder, 1893) & GreR & P2i-1 & 77 \\
\hline 158 & A. provectus (Scudder, 1876) & GreR & P2i-1 & 73 \\
\hline 159 & A. scudderi Legalov, 2018 & GreR & P2i-1 & 69 \\
\hline 160 & A. ovalis Legalov, 2018 & GreR & P2i-1 & 69 \\
\hline 161 & A. deleticius (Scudder, 1893) & WhiR & $\mathrm{P} 3 \mathrm{r}$ & 77 \\
\hline 162 & A. lacoei (Scudder, 1893) & Flor & $\mathrm{P} 2 \mathrm{p}$ & 77 \\
\hline 163 & "Anisorhynchus" offosus Oustalet, 1870 & Core & $\mathrm{P} 3 \mathrm{r}$ & 119 \\
\hline 164 & "Hylobius" antiquus Heyden et Heyden, 1866 & Rott & P3h & 85 \\
\hline \multirow{2}{*}{\multicolumn{5}{|c|}{$\begin{array}{l}\text { Plinthini Lacordaire, } 1863 \\
\text { Leiosomatina Reitter, } 1913\end{array}$}} \\
\hline & & & & \\
\hline 165 & Leiosoma klebsi Legalov, 2016 & BalJ & $\mathrm{P} 2 \mathrm{~b}$ & 115 \\
\hline \multicolumn{5}{|c|}{ Pissodini Gistel, 1856} \\
\hline 166 & Lithopissodes luschitzensis Beier, 1952 & Luzi & P3h & 120 \\
\hline \multicolumn{5}{|c|}{ Acicnemidini Lacordaire, 1865} \\
\hline 167 & Electrotribus henningseni (Voss, 1972) & $\begin{array}{c}\text { ScanJ, PolJ } \\
\text { BalJ, PolJ }\end{array}$ & $\mathrm{P} 2 \mathrm{~b}$ & 105 \\
\hline \multirow[t]{4}{*}{168} & E. theryi Hustache, 1942 & & $\mathrm{P} 2 \mathrm{~b}$ & 121 \\
\hline & =Paleopissodes weigangae Ulke, 1947 & & & 122 \\
\hline & =Anchorthorrhinus incertus Voss, 1953 & & & 80 \\
\hline & =Isalcidodes Macellus Voss, 1953 & BalJ & & 80 \\
\hline 169 & E. wolfschwenningerae (Rheinheimer, 2007) & BalJ & $\mathrm{P} 2 \mathrm{~b}$ & 102 \\
\hline 170 & E. rarus Legalov, 2020 & & $\mathrm{P} 2 \mathrm{~b}$ & 71 \\
\hline \multicolumn{5}{|c|}{ Magdalini Pascoe, 1870} \\
\hline 171 & "Magdalis" sedimentorum Scudder, 1893 & Flor & $\mathrm{P} 2 \mathrm{p}$ & 77 \\
\hline 172 & “M." moesta Schlechtendal, 1894 & Rott & P3h & 89 \\
\hline 173 & "M." deucalionis (Heyden et Heyden, 1866) & Rott & P3h & 85 \\
\hline 174 & “M.“ protogenius (Heyden et Heyden, 1866) & Rott & P3h & 85 \\
\hline \multicolumn{5}{|c|}{ Cleogonini Gistel, 1856} \\
\hline 175 & Rhysosternum aeternabile Scudder, 1893 & Flor & $\mathrm{P} 2 \mathrm{p}$ & 77 \\
\hline 176 & Rh. longirostre Scudder, 1893 & Flor & $\mathrm{P} 2 \mathrm{p}$ & 77 \\
\hline 177 & "Conotrachelus" florissantensis Wickham, 1912 & Flor & $\mathrm{P} 2 \mathrm{p}$ & 102 \\
\hline
\end{tabular}


Table 2. Cont.

\begin{tabular}{|c|c|c|c|c|}
\hline No. & Taxon & Locality & Age & Sources \\
\hline \multicolumn{5}{|c|}{ Sciabregmini Legalov, Kirejtshuk et Nel, 2019} \\
\hline 178 & Sciabregma rugosa Scudder, 1893 & RoaM & P2i-1 & 77 \\
\hline 179 & S. squamosa Legalov, Kirejtshuk et Nel, 2019 & OisJ & $\mathrm{P} 2 \mathrm{i}$ & 81 \\
\hline 180 & S. tenuicornis Cockerell, 1921 & GreR & P2i-1 & 123 \\
\hline \multicolumn{5}{|c|}{ Camptorhinini Lacordaire, 1865} \\
\hline 181 & Camptorrhinites orarius Britton, 1960 & LonC & $\mathrm{P} 2 \mathrm{i}$ & 114 \\
\hline 182 & Korystina gracilis Britton, 1960 & LonC & $\mathrm{P} 2 \mathrm{i}$ & 114 \\
\hline \multicolumn{5}{|c|}{ Aedemonini Faust, 1898} \\
\hline 183 & Electrorhinus friedhelmi Legalov, 2020 & BalJ & $\mathrm{P} 2 \mathrm{~b}$ & 71 \\
\hline \multicolumn{5}{|c|}{$\begin{array}{l}\text { Cryptorhynchini Schoenherr, } 1825 \\
\text { Cryptorhynchina Schoenherr, } 1825\end{array}$} \\
\hline 184 & Taylorius litoralis Britton, 1960 & LonC & $\mathrm{P} 2 \mathrm{i}$ & 114 \\
\hline 185 & "Cryptorhynchus" annosus Scudder, 1876 & GreR & P2i-1 & 73 \\
\hline 186 & "C." coloradensis Wickham, 1912 & Flor & $\mathrm{P} 2 \mathrm{p}$ & 102 \\
\hline 187 & “C." evinctus Scudder, 1893 & Flor & $\mathrm{P} 2 \mathrm{p}$ & 77 \\
\hline 188 & “C.” falli Wickham, 1912 & Flor & $\mathrm{P} 2 \mathrm{p}$ & 102 \\
\hline 189 & "C." kerri Scudder, 1893 & Flor & $\mathrm{P} 2 \mathrm{p}$ & 77 \\
\hline 190 & "C." profusus Scudder, 1893 & Flor & $\mathrm{P} 2 \mathrm{p}$ & 77 \\
\hline 191 & Oisecalles latosquamosus Legalov, Kirejtshuk et Nel, 2019 & OisJ & $\mathrm{P} 2 \mathrm{i}$ & 81 \\
\hline 192 & Cryptorrhynchites sculpturatus Haupt, 1950 & Geis & P21 & 124 \\
\hline 193 & Succinacalles uniqus Zherikhin, 1971 & BalJ & $\mathrm{P} 2 \mathrm{~b}$ & 79 \\
\hline \multicolumn{5}{|c|}{ Tylodina Lacordaire, 1865} \\
\hline 194 & Baltacalles triumurbium Bukejs, Alekseev et Legalov, 2020 & BalJ & $\mathrm{P} 2 \mathrm{~b}$ & 125 \\
\hline 195 & “Acalles" icarus Heyden et Heyden, 1866 & Rott & P3h & 85 \\
\hline \multicolumn{5}{|c|}{ Tribe incertae sedis } \\
\hline 196 & Lutago fetosus Britton, 1960 & LonC & $\mathrm{P} 2 \mathrm{i}$ & 114 \\
\hline 197 & L. nanus Britton, 1960 & LonC & $\mathrm{P} 2 \mathrm{i}$ & 114 \\
\hline 198 & Pissodites argillosus Britton, 1960 & LonC & $\mathrm{P} 2 \mathrm{i}$ & 114 \\
\hline 199 & "Chalcodermus" kirschi Deichmueller, 1881 & Kuts & $\mathrm{P} 2 \mathrm{~b}$ & 126 \\
\hline 200 & Laccopygus nilesii Scudder, 1893 & Flor & $\mathrm{P} 2 \mathrm{p}$ & 77 \\
\hline 201 & L. rhenanus Meunier, 1924 & Rott & P3h & 127 \\
\hline 202 & "Acalles" exhumatus Wickham, 1913 & Flor & $\mathrm{P} 2 \mathrm{p}$ & 97 \\
\hline 203 & “Pissodes" planatus Foerster, 1891 & Brun & $\mathrm{P} 3 \mathrm{r}$ & 87 \\
\hline 204 & "Hylobius" deletus Oustalet, 1870 & Core & P3r & 119 \\
\hline 205 & "Plinthus" redivivus Oustalet, 1870 & Core & $\mathrm{P} 3 \mathrm{r}$ & 119 \\
\hline 206 & "Hylobius" morosus Oustalet, 1874 & Core & P3r & 110 \\
\hline 207 & "Molytes" hassencampi Heyden, 1858 & Sieb & P3 & 128 \\
\hline 208 & "Pissodes" effossus Heyden, 185 & Sieb & P3 & 128 \\
\hline 209 & $\begin{array}{c}8 \\
\text { “Hylobius" carbo Oustalet, } 1874\end{array}$ & Aix & P3h & 110 \\
\hline 210 & "Plinthus" heerii Oustalet, 1874 & Aix & P3h & 110 \\
\hline \multicolumn{5}{|c|}{$\begin{array}{l}\text { Lixinae Schoenherr, } 1823 \\
\text { Lixini Schoenherr, } 1823\end{array}$} \\
\hline 212 & Lixus ligniticus Piton, 1940 & Mena & P1sl-t & 99 \\
\hline 213 & "Larinus" longirostris Foerster, 1891 & Brun & $\mathrm{P} 3 \mathrm{r}$ & 87 \\
\hline 214 & “L.“ bronni Heyden et Heyden, 1866 & Rott & P3h & 85 \\
\hline
\end{tabular}


Table 2. Cont.

\begin{tabular}{|c|c|c|c|c|}
\hline No. & Taxon & Locality & Age & Sources \\
\hline 215 & Eocleonus subjectus Scudder, 1893 & Flor & $\mathrm{P} 2 \mathrm{p}$ & 77 \\
\hline 216 & "Cleonus" degenerates Scudder, 1893 & Flor & $\mathrm{P} 2 \mathrm{p}$ & 77 \\
\hline 217 & “C." estriatus Wickham, 1912 & Flor & $\mathrm{P} 2 \mathrm{p}$ & 102 \\
\hline 218 & "C." exterraneus Scudder, 1893 & Flor & $\mathrm{P} 2 \mathrm{p}$ & 77 \\
\hline 219 & “C.” foersteri Scudder, 1893 & Flor & $\mathrm{P} 2 \mathrm{p}$ & 77 \\
\hline 220 & “C." primoris Scudder, 1893 & Flor & $\mathrm{P} 2 \mathrm{p}$ & 77 \\
\hline 221 & “C." rohweri Wickham, 1911 & Flor & $\mathrm{P} 2 \mathrm{p}$ & 76 \\
\hline 222 & "C." arvernensis Oustalet, 1870 & Core & $\mathrm{P} 3 \mathrm{r}$ & 119 \\
\hline 223 & “C." fouilhouxii Oustalet, 1870 & Core & $\mathrm{P} 3 \mathrm{r}$ & 119 \\
\hline \multicolumn{5}{|c|}{$\begin{array}{l}\text { Dryophthorinae Schoenherr, } 1825 \\
\text { Stromboscerini Lacordaire, } 1865\end{array}$} \\
\hline 224 & Palaeodexipeus kirejtshuki Legalov, 2016 & BalJ & $\mathrm{P} 2 \mathrm{~b}$ & 90 \\
\hline 225 & $\begin{array}{l}\text { Rovnoslonik damzeni Legalov, Nazarenko et Perkovsky, } \\
2019\end{array}$ & RovJ & $\mathrm{P} 2 \mathrm{~b}$ & 117 \\
\hline 226 & Stenommatomorphus hexarthrus Nazarenko, 2009 & RovJ & $\mathrm{P} 2 \mathrm{~b}$ & 129 \\
\hline \multicolumn{5}{|c|}{ Dryophthorini Schoenherr, 1825} \\
\hline 227 & Rhinoporkus gratiosus Legalov, Kirejtshuk et Nel, 2019 & OisJ & $\mathrm{P} 2 \mathrm{i}$ & 81 \\
\hline 228 & Lithophthorus rugosicollis Scudder, 1893 & Flor & $\mathrm{P} 2 \mathrm{p}$ & 77 \\
\hline 229 & Spodotribus terrulentus Scudder, 1893 & Flor & $\mathrm{P} 2 \mathrm{p}$ & 77 \\
\hline 230 & Dryophthorus incertus (Theobald, 1935) & Aix & P3h & 130 \\
\hline \multicolumn{5}{|c|}{$\begin{array}{l}\text { Sphenophorini Lacordaire, } 1865 \\
\text { Sphenophorina Lacordaire, } 1865\end{array}$} \\
\hline 231 & "Scyphophorus" fossionis Scudder, 1893 & Flor & $\mathrm{P} 2 \mathrm{p}$ & 77 \\
\hline 232 & S. laevis Scudder, 1893 & Flor & $\mathrm{P} 2 \mathrm{p}$ & 77 \\
\hline 233 & "S." tertiarius Wickham, 1911 & Flor & $\mathrm{P} 2 \mathrm{p}$ & 76 \\
\hline 234 & Oryctorhinus tenuirostris Scudder, 1893 & Flor & $\mathrm{P} 2 \mathrm{p}$ & 77 \\
\hline 235 & “Sphenophorus" proluviosus Heyden et Heyden, 1866 & Rott & $\mathrm{P} 3 \mathrm{~h}$ & 85 \\
\hline \multicolumn{5}{|c|}{ Tribe incertae sedis } \\
\hline 236 & Hipporhinops sternbergi Cockerell, 1926 & Flor & $\mathrm{P} 2 \mathrm{p}$ & 131 \\
\hline \multicolumn{5}{|c|}{$\begin{array}{c}\text { Cossoninae Schoenherr, } 1825 \\
\text { Dryotribini LeConte, } 1876\end{array}$} \\
\hline 237 & Ampharthropelma decipiens Voss, 1972 & ScanJ & $\mathrm{P} 2 \mathrm{~b}$ & 105 \\
\hline 238 & $\begin{array}{l}\text { Caulophilus Martynovae Legalov, Nazarenko et Perkovsky, } \\
\qquad 2019\end{array}$ & RovJ & $\mathrm{P} 2 \mathrm{~b}$ & 117 \\
\hline 239 & C. rarus Legalov, 2016 & BalJ & $\mathrm{P} 2 \mathrm{~b}$ & 115 \\
\hline 240 & C. squamosus Legalov, 2016 & BalJ & $\mathrm{P} 2 \mathrm{~b}$ & 115 \\
\hline 241 & C. sucinopunctatus (Kuska, 1992) & BalJ & $\mathrm{P} 2 \mathrm{~b}$ & 132 \\
\hline 242 & C. zherikhini Nazarenko, Legalov et Perkovsky, 2011 & RovJ & $\mathrm{P} 2 \mathrm{~b}$ & 133 \\
\hline 243 & Necrodryophthorus inquilinus Voss, 1953 & BalJ & $\mathrm{P} 2 \mathrm{~b}$ & 80 \\
\hline 244 & Synommatodes patruelis (Voss, 1953) & BalJ & $\mathrm{P} 2 \mathrm{~b}$ & 80 \\
\hline 245 & Electrocossonus kirejtshuki Legalov, 2020 & BalJ & $\mathrm{P} 2 \mathrm{~b}$ & 71 \\
\hline \multicolumn{5}{|c|}{$\begin{array}{l}\text { Rhyncolini Gistel, } 1856 \\
\text { Rhyncolina Gistel, } 1856\end{array}$} \\
\hline 246 & Rhyncolus sitonifrons Zherikhin, 1992 & Rott & P3h & 98 \\
\hline \multicolumn{5}{|c|}{ Cossonini Schoenherr, 1825} \\
\hline 247 & "Cossonus" devoratus Cockerell, 1925 & Sunc & P1d & 134 \\
\hline 248 & “C." rutus Scudder, 1893 & RoaM & P2i-1 & 77 \\
\hline 249 & "C." gabbii Scudder, 1893 & Flor & $\mathrm{P} 2 \mathrm{p}$ & 77 \\
\hline 250 & C. robustus Meunier, 1916 & Aix & P3h & 135 \\
\hline
\end{tabular}


Table 2. Cont.

\begin{tabular}{|c|c|c|c|c|}
\hline No. & Taxon & Locality & Age & Sources \\
\hline \multicolumn{5}{|c|}{$\begin{array}{c}\text { Conoderinae Schoenherr, } 1833 \\
\text { Bariditae Schoenherr, } 1836 \\
\text { Apostasimerini Schoenherr, } 1844 \\
\text { Coelonertina Casey, } 1922\end{array}$} \\
\hline 251 & Geraeus diruptus (Scudder, 1893) & GreR & P2i-1 & 77 \\
\hline 252 & G. anvilis Legalov, 2018 & GreR & P2i-1 & 70 \\
\hline 253 & G. fossilis Legalov, 2018 & GreR & P2i-1 & 70 \\
\hline 254 & G. antediluviana (Wickham, 1916) & Flor & $\mathrm{P} 2 \mathrm{p}$ & 100 \\
\hline 255 & G. hoveyi (Wickham, 1912) & Flor & $\mathrm{P} 2 \mathrm{p}$ & 101 \\
\hline 256 & G. hypogaeus (Wickham, 1916) & Flor & $\mathrm{P} 2 \mathrm{p}$ & 100 \\
\hline 257 & G. matura (Scudder, 1893) & Flor & $\mathrm{P} 2 \mathrm{p}$ & 77 \\
\hline 258 & G. obnuptus (Scudder, 1893) & Flor & P2p & 77 \\
\hline 259 & G. schucherti (Wickham, 1912) & Flor & $\mathrm{P} 2 \mathrm{p}$ & 101 \\
\hline 260 & G. vulcanicus (Wickham, 1913) & Flor & $\mathrm{P} 2 \mathrm{p}$ & 97 \\
\hline 261 & Lithogeraeus greenriverensis Legalov, 2018 & GreR & P2i-1 & 70 \\
\hline 262 & L. circumscripta (Scudder, 1893) & RoaM & P2i-1 & 77 \\
\hline 263 & L. ancilla (Scudder, 1893) & RoaM & P2i-1 & 77 \\
\hline 264 & L. comminute (Scudder, 1893) & WhiR & $\mathrm{P} 3 \mathrm{r}$ & 77 \\
\hline 265 & L. damnata (Scudder, 1893) & Flor & $\mathrm{P} 2 \mathrm{p}$ & 77 \\
\hline 266 & L. cremastorhynchoides (Wickham, 1913) & Flor & P2p & 97 \\
\hline 267 & L. florissantensis (Wickham, 1913) & Flor & $\mathrm{P} 2 \mathrm{p}$ & 97 \\
\hline 268 & L. nearctica (Wickham, 1916) & Flor & $\mathrm{P} 2 \mathrm{p}$ & 100 \\
\hline 269 & L. primalis (Wickham, 1917) & Flor & P2p & 136 \\
\hline 270 & L. renovata (Wickham, 1916) & Flor & $\mathrm{P} 2 \mathrm{p}$ & 100 \\
\hline 271 & Nicentrus curvirostris Legalov, 2018 & GreR & P2i-1 & 70 \\
\hline 272 & Steganus barrandei Scudder, 1893 & RoaM & P2i-1 & 77 \\
\hline 273 & Miogeraeus recurrens Wickham, 1916 & Flor & $\mathrm{P} 2 \mathrm{p}$ & 100 \\
\hline 274 & "Pachybaris" rudis Wickham, 1912 & Flor & $\mathrm{P} 2 \mathrm{p}$ & 96 \\
\hline \multicolumn{5}{|c|}{$\begin{array}{l}\text { Baridini Schoenherr, } 1836 \\
\text { Baridina Schoenherr, } 1836\end{array}$} \\
\hline 275 & "Baris" palaeophilus Cockerell, 1920 & Bour & P21 & 137 \\
\hline 276 & "B." divisa Scudder, 1893 & Flor & $\mathrm{P} 2 \mathrm{p}$ & 77 \\
\hline 277 & "B." harlani Scudder, 1893 & Flor & $\mathrm{P} 2 \mathrm{p}$ & 77 \\
\hline 278 & "B." imperfecta Scudder, 1893 & Flor & P2p & 77 \\
\hline 279 & "B." naviculare (Foerster, 1891) & Brun & $\mathrm{P} 3 \mathrm{r}$ & 87 \\
\hline 280 & Catobaris coenosa Scudder, 1893 & Flor & $\mathrm{P} 2 \mathrm{p}$ & 77 \\
\hline \multicolumn{5}{|c|}{ Eurhinina Lacordaire, 1865} \\
\hline 281 & Eurhinus occultus Scudder, 1876 & Flor & $\mathrm{P} 2 \mathrm{p}$ & 77 \\
\hline \multicolumn{5}{|c|}{ Tribe incertae sedis } \\
\hline 282 & “Ceutorhynchus" clausus Scudder, 1893 & Flor & $\mathrm{P} 2 \mathrm{p}$ & 77 \\
\hline \multicolumn{5}{|c|}{$\begin{array}{c}\text { Conoderintae Schoenherr, } 1833 \\
\text { Conoderini Schoenherr, } 1833\end{array}$} \\
\hline 283 & Jantarhinus compressus Legalov, Kirejtshuk et Nel, 2019 & OisJ & $\mathrm{P} 2 \mathrm{i}$ & 81 \\
\hline \multicolumn{5}{|c|}{ Palaeomallerini Legalov, 2018} \\
\hline 284 & Palaeomallerus longirostris Legalov, 2018 & GreR & P2i-1 & 70 \\
\hline & $\begin{array}{l}\text { Ceutorhynchitae Gistel, } 1848 \\
\text { Ceutorhynchini Gistel, } 1848\end{array}$ & & & \\
\hline
\end{tabular}


Table 2. Cont.

\begin{tabular}{|c|c|c|c|c|}
\hline No. & Taxon & Locality & Age & Sources \\
\hline 285 & “Ceutorhynchus" degravatus Scudder, 1893 & RoaM & P2i-1 & 77 \\
\hline 286 & "C." eocenicus Cockerell, 1920 & Peck & $\mathrm{P} 2 \mathrm{i}$ & 137 \\
\hline 287 & C. alekseevi Legalov, 2016 & BalJ & $\mathrm{P} 2 \mathrm{~b}$ & 115 \\
\hline 288 & C. electrinus Legalov, 2016 & BalJ & $\mathrm{P} 2 \mathrm{~b}$ & 115 \\
\hline 289 & C. succinus Legalov, 2013 & BalJ & $\mathrm{P} 2 \mathrm{~b}$ & 47 \\
\hline 290 & “C." blaisdelli Wickham, 1916 & Flor & $\mathrm{P} 2 \mathrm{p}$ & 100 \\
\hline 291 & "C." compactus Scudder, 1893 & Flor & $\mathrm{P} 2 \mathrm{p}$ & 77 \\
\hline 292 & “C." duratus Scudder, 1893 & Flor & $\mathrm{P} 2 \mathrm{p}$ & 77 \\
\hline 293 & “C." irvingi (Scudder, 1893) & Flor & $\mathrm{P} 2 \mathrm{p}$ & 77 \\
\hline 294 & "C." fischeri Foerster, 1891 & Brun & $\mathrm{P} 3 \mathrm{r}$ & 87 \\
\hline 295 & "C." crassirostris Foerster, 1891 & Brun & $\mathrm{P} 3 \mathrm{r}$ & 87 \\
\hline 296 & "C." obliquus Foerster, 1891 & Brun & $\mathrm{P} 3 \mathrm{r}$ & 87 \\
\hline 297 & “C." funeratus Heyden et Heyden, 1866 & Rott & P3h & 85 \\
\hline 298 & Baltocoeliodes sontagae Legalov et Bukejs, 2018 & BalJ & $\mathrm{P} 2 \mathrm{~b}$ & 138 \\
\hline \multicolumn{5}{|c|}{ Cnemogonini Colonnelli, 1979} \\
\hline 299 & “Coeliodes" primotinus Scudder, 1893 & Flor & $\mathrm{P} 2 \mathrm{p}$ & 77 \\
\hline \multicolumn{5}{|c|}{ Phytobiini Gistel, 1848} \\
\hline 300 & "Ceuthorrhynchus"miegi Theobald, 1937 & Klei & P3 & 112 \\
\hline \multicolumn{5}{|c|}{$\begin{array}{l}\text { Curculioninae Latreille, } 1802 \\
\text { Acalyptini C.G. Thomson, } 1859\end{array}$} \\
\hline 301 & Jantaronosik nebulosus Legalov, Kirejtshuk et Nel, 2019 & OisJ & $\mathrm{P} 2 \mathrm{i}$ & 81 \\
\hline \multicolumn{5}{|c|}{ Ellescini C.G. Thomson, 1859} \\
\hline 302 & Succinostyphlus mroczkowskii Kuska, 1996 & BalJ & $\mathrm{P} 2 \mathrm{~b}$ & 139 \\
\hline & $=$ Electrotribus erectosquamata Rheinheimer, 2007 & & & 122 \\
\hline 303 & Pachytychius eocenicus Legalov, 2016 & BalJ & $\mathrm{P} 2 \mathrm{~b}$ & 115 \\
\hline \multicolumn{5}{|c|}{ Palaeoanoplini Legalov, in print. } \\
\hline 304 & Palaeoanoplus horridus Legalov, in print. & BalJ & $\mathrm{P} 2 \mathrm{~b}$ & 140 \\
\hline \multicolumn{5}{|c|}{ Smicronychini Seidlitz, 1891} \\
\hline 305 & "Smicronyx" antiquus Foerster, 1891 & Brun & $\mathrm{P} 3 \mathrm{r}$ & 87 \\
\hline \multicolumn{5}{|c|}{$\begin{array}{c}\text { Curculionini Latreille, } 1802 \\
\text { Erganiina Pelsue et O’Brien, } 2011\end{array}$} \\
\hline 306 & Pseudoergania perkovskyi Legalov, 2019 & BalJ & $\mathrm{P} 2 \mathrm{~b}$ & 141 \\
\hline \multicolumn{5}{|c|}{ Timolina Pelsue et O'Brien, 2011} \\
\hline 307 & Baltocurculio Manukyani Legalov, 2020 & BalJ & $\mathrm{P} 2 \mathrm{~b}$ & 71 \\
\hline \multicolumn{5}{|c|}{ Curculionina Latreille, 1802} \\
\hline 308 & Menatorhis elegans (Piton, 1940) & Mena & P1sl- & 99 \\
\hline 309 & Curculio havighorstensis Zherikhin, 1995 & Havi & $\mathrm{tP} 2 \mathrm{i}$ & 142 \\
\hline 310 & C. anicularis (Scudder, 1893) & Flor & $\mathrm{P} 2 \mathrm{p}$ & 77 \\
\hline 311 & C. curvirostris (Scudder, 1893) & Flor & $\mathrm{P} 2 \mathrm{p}$ & 77 \\
\hline 312 & C. duttoni (Scudder, 1893) & Flor & $\mathrm{P} 2 \mathrm{p}$ & 77 \\
\hline 313 & C. extinctus (Wickham, 1912) & Flor & $\mathrm{P} 2 \mathrm{p}$ & 102 \\
\hline 314 & C. femoratus (Scudder, 1893) & Flor & $\mathrm{P} 2 \mathrm{p}$ & 77 \\
\hline 315 & C. flexirostris (Scudder, 1893) & Flor & $\mathrm{P} 2 \mathrm{p}$ & 77 \\
\hline 316 & C. florissantensis (Wickham, 1913) & Flor & $\mathrm{P} 2 \mathrm{p}$ & 97 \\
\hline 317 & C. minusculoides (Wickham, 1911) & Flor & $\mathrm{P} 2 \mathrm{p}$ & 76 \\
\hline 318 & C. minusculus (Scudder, 1893) & Flor & $\mathrm{P} 2 \mathrm{p}$ & 77 \\
\hline 319 & C. restrictus (Scudder, 1893) & Flor & $\mathrm{P} 2 \mathrm{p}$ & 77 \\
\hline 320 & “C.” beeklyi (Cockerell, 1918) & Flor & $\mathrm{P} 2 \mathrm{p}$ & 143 \\
\hline 321 & Numitor claviger Scudder, 1893 & Flor & $\mathrm{P} 2 \mathrm{p}$ & 77 \\
\hline
\end{tabular}


Table 2. Cont.

\begin{tabular}{|c|c|c|c|c|}
\hline No. & Taxon & Locality & Age & Sources \\
\hline \multicolumn{5}{|c|}{ Anthonomini C.G. Thomson, 1859} \\
\hline 322 & "Anthonomus" sunchalensis Cockerell, 1925 & Sunc & P1d & 134 \\
\hline \multirow[t]{2}{*}{323} & “A.” soporus Scudder, 1890 & $\begin{array}{l}\text { GreR, RoaM, } \\
\text { WhiR }\end{array}$ & P2i-1 & 78 \\
\hline & & $\begin{array}{l}\text { GreR } \\
\text { Flor }\end{array}$ & $\mathrm{P} 3 \mathrm{r}$ & \\
\hline 324 & "A." revictus Scudder, 1893 & Flor & $\mathrm{P} 2 \mathrm{i}-1$ & 77 \\
\hline 325 & "A." concussus Scudder, 1893 & Flor & $\mathrm{P} 2 \mathrm{p}$ & 77 \\
\hline 326 & “A." debilatus Scudder, 1893 & Flor & $\mathrm{P} 2 \mathrm{p}$ & 77 \\
\hline 327 & "A." defossus Scudder, 1893 & Flor & $\mathrm{P} 2 \mathrm{p}$ & 77 \\
\hline 328 & "A." evigilatus Scudder, 1893 & Flor & $\mathrm{P} 2 \mathrm{p}$ & 77 \\
\hline 329 & "A." primordius Scudder, 1893 & Flor & $\mathrm{P} 2 \mathrm{p}$ & 77 \\
\hline 330 & "A." rohweri Wickham, 1912 & Flor & $\mathrm{P} 2 \mathrm{p}$ & 96 \\
\hline 331 & Coccotorus principalis Scudder, 1893 & Flor & $\mathrm{P} 2 \mathrm{p}$ & 77 \\
\hline 332 & “C." requiescens Scudder, 1893 & Flor & $\mathrm{P} 2 \mathrm{p}$ & 77 \\
\hline 333 & Cremastorhynchus stabilis Scudder, 1893 & & $\mathrm{P} 2 \mathrm{p}$ & 77 \\
\hline 334 & Smicrorhynchus Macgeei Scudder, 1893 & & $\mathrm{P} 2 \mathrm{p}$ & 77 \\
\hline \multicolumn{5}{|c|}{ Eugnomini Lacordaire, 1863} \\
\hline 335 & Archaeoeugnomus balticus Legalov, 2016 & BalJ & $\mathrm{P} 2 \mathrm{~b}$ & 115 \\
\hline 336 & Anthonoeugnomus barsevskisi Legalov, 2020 & BalJ & $\mathrm{P} 2 \mathrm{~b}$ & 71 \\
\hline 337 & Mazurieugnomus pilosus Legalov, 2020 & BalJ & $\mathrm{P} 2 \mathrm{~b}$ & 71 \\
\hline 338 & Groehnius electrum Bukejs et Legalov, 2019 & BalJ & $\mathrm{P} 2 \mathrm{~b}$ & 144 \\
\hline 339 & G. parvum Legalov, 2020 & BalJ & $\mathrm{P} 2 \mathrm{~b}$ & 71 \\
\hline \multicolumn{5}{|c|}{$\begin{array}{l}\text { Rhamphini Rafinesque, } 1815 \\
\text { Palaeorhamphina Legalov, } 2016\end{array}$} \\
\hline 340 & Palaeorhamphus damzeni Legalov, 2020 & BalJ & $\mathrm{P} 2 \mathrm{~b}$ & 71 \\
\hline 341 & P. eichmanni Legalov, 2020 & BalJ & $\mathrm{P} 2 \mathrm{~b}$ & 71 \\
\hline 342 & P. primitivus Legalov, 2016 & BalJ & $\mathrm{P} 2 \mathrm{~b}$ & 115 \\
\hline \multicolumn{5}{|c|}{ Rhamphina Rafinesque, 1815} \\
\hline 343 & Orchestes tatjanae Legalov, 2016 & BalJ & $\mathrm{P} 2 \mathrm{~b}$ & 115 \\
\hline 344 & “O.” languidulus Scudder, 1893 & Flor & $\mathrm{P} 2 \mathrm{p}$ & 77 \\
\hline 345 & Tachyerges hyperoche Legalov et Poinar, 2020 & PolJ & $\mathrm{P} 2 \mathrm{~b}$ & 91 \\
\hline \multirow{2}{*}{\multicolumn{5}{|c|}{$\begin{array}{l}\text { Tychiini C.G. Thomson, } 1859 \\
\text { Tychiina C.G. Thomson, } 1859\end{array}$}} \\
\hline & & & & \\
\hline 346 & Eocenesibinia prussica Legalov, 2016 & BalJ & $\mathrm{P} 2 \mathrm{~b}$ & 115 \\
\hline 347 & "Sibinia" whitneyi (Scudder, 1893) & Flor & $\mathrm{P} 2 \mathrm{p}$ & 77 \\
\hline 348 & "S." melancholicus (Oustalet, 1874) & Aix & P3h & 110 \\
\hline 349 & "Tychius" evolatus Scudder, 1893 & Flor & $\mathrm{P} 2 \mathrm{p}$ & 77 \\
\hline 350 & "T." ferox Wickham, 1917 & Flor & $\mathrm{P} 2 \mathrm{p}$ & 136 \\
\hline 351 & "T." secretus Scudder, 1893 & Flor & $\mathrm{P} 2 \mathrm{p}$ & 77 \\
\hline 352 & “T.” latus Foerster, 1891 & Brun & $\mathrm{P} 3 \mathrm{r}$ & 87 \\
\hline 353 & “T." manderstjernai Heyden et Heyden, 1866 & Rott & P3h & 85 \\
\hline 354 & Macrorhoptus intutus Scudder, 1893 & Flor & $\mathrm{P} 2 \mathrm{p}$ & 77 \\
\hline \multicolumn{5}{|c|}{$\begin{array}{l}\text { Camarotini Schoenherr, } 1833 \\
\text { Prionomerina Lacordaire, } 1863\end{array}$} \\
\hline 355 & Paleodontopus smirnovae Legalov, 2020 & BalJ & $\mathrm{P} 2 \mathrm{~b}$ & 71 \\
\hline 356 & Masteutes rupis Scudder, 1893 & Flor & $\mathrm{P} 2 \mathrm{p}$ & 77 \\
\hline \multicolumn{5}{|c|}{ Curculioninae incertae sedis } \\
\hline 357 & "Centrinus" longipes Piton, 1940 & Mena & P1sl-t & 99 \\
\hline 358 & "Gymnetron" lecontei Scudder, 1878 & GreR & P2i-1 & 74 \\
\hline 359 & "G." antecurrens Scudder, 1893 & Flor RovJ & $\mathrm{P} 2 \mathrm{p}$ & 77 \\
\hline 360 & "Protoceletes" hirtus Nazarenko et Perkovsky, 2016 & & $\mathrm{P} 2 \mathrm{~b}$ & 145 \\
\hline
\end{tabular}


Table 2. Cont

\begin{tabular}{|c|c|c|c|c|}
\hline No. & Taxon & Locality & Age & Sources \\
\hline \multirow{3}{*}{\multicolumn{5}{|c|}{$\begin{array}{c}\text { Cyclominae Schoenherr, } 1826 \\
\text { Listroderini LeConte, } 1876 \\
\text { Palaechthina Brinck, } 1948\end{array}$}} \\
\hline & & & & \\
\hline & & & & \\
\hline 361 & "Listronotus" muratus Scudder, 1890 & GreR & P2i-1 & 78 \\
\hline \multicolumn{5}{|c|}{ Listroderina LeConte, 1876} \\
\hline 362 & "Listroderes" differens (Wickham, 1912) & Flor & P2p & 102 \\
\hline 363 & “L." eviscerates (Scudder, 1893) & Flor & $\mathrm{P} 2 \mathrm{p}$ & 77 \\
\hline \multicolumn{5}{|c|}{ Entiminae Schoenherr, 1823} \\
\hline \multicolumn{5}{|c|}{ Entimintae Schoenherr, 1823} \\
\hline \multicolumn{5}{|c|}{ Tropiphorini Marseul, 1863} \\
\hline 364 & Primocentron wickhami Legalov, 2018 & GreR & P2i-1 & 69 \\
\hline 365 & Limalophus poinari Legalov, 2020 & PolJ & $\mathrm{P} 2 \mathrm{~b}$ & 71 \\
\hline 366 & Scuccinalophus attenboroughi Legalov, 2016 & BalJ & $\mathrm{P} 2 \mathrm{~b}$ & 90 \\
\hline 367 & Limalophus compositus Scudder, 1893 & GreR, WhiR & P2i-1, & 77 \\
\hline & & GreR & $\mathrm{P} 3 \mathrm{r}$ & \\
\hline 368 & L. contractus Scudder, 1893 & WhiR & P2i-1 & 77 \\
\hline 369 & "L." receptus Scudder, 1893 & WhiR & P3r & 77 \\
\hline 370 & "Coniatus" refractus Scudder, 1893 & RoaM & $\mathrm{P} 3 \mathrm{r}$ & 77 \\
\hline 371 & "Cryptorhynchus" durus Scudder, 1893 & GreR & P2i-1 & 77 \\
\hline 372 & "Lepyrus" evictus Scudder, 1893 & GreR, RoaM & P2 i-1 & 77 \\
\hline 373 & "Sitona" paginarum Scudder, 1893 & RoaM & P2 i-1 & 77 \\
\hline 374 & & RoaM GreR & & \\
\hline 375 & “Otiorhynchus" subteractus Scudder, 1893 & RoaM & P2 i-1 & 77 \\
\hline 376 & “Ophryastes" grandis Scudder, 1893 & Flor & P2 i-1 & 77 \\
\hline 377 & "O." compactus Scudder, 1878 & Flor & P2 i-1 & 77 \\
\hline 378 & “O." petrarum Scudder, 1893 & Flor & P2 i-1 & 77 \\
\hline 379 & "O." championi Wickham, 1912 & Flor & $\mathrm{P} 2 \mathrm{p}$ & 102 \\
\hline 380 & Geralophus antiquarius Scudder, 1893 & Flor & $\mathrm{P} 2 \mathrm{p}$ & 77 \\
\hline 381 & G. fossicius Scudder, 1893 & Flor & $\mathrm{P} 2 \mathrm{p}$ & 77 \\
\hline 382 & G. lassatus Scudder, 1893 & Flor & $\mathrm{P} 2 \mathrm{p}$ & 77 \\
\hline 383 & G. occultus Scudder, 1893 & Flor & $\mathrm{P} 2 \mathrm{p}$ & 77 \\
\hline \multirow[t]{2}{*}{384} & G. pumiceus Scudder, 1893 & Flor & $\mathrm{P} 2 \mathrm{p}$ & 77 \\
\hline & G. repositus Scudder, 1893 & Flor & $\mathrm{P} 2 \mathrm{p}$ & 77 \\
\hline 385 & G. retritus Scudder, 1893 & Flor & P2p & 77 \\
\hline 386 & G. saxuosus Scudder, 1893 & Flor & P2p & 77 \\
\hline 387 & G. scudderi Wickham, 1911 & Aix & $\mathrm{P} 2 \mathrm{p}$ & 76 \\
\hline 388 & Centron moricollis Scudder, 1893 & & $\mathrm{P} 2 \mathrm{p}$ & 77 \\
\hline 389 & Tenillus firmus Scudder, 1893 & & P2p & 77 \\
\hline \multirow[t]{3}{*}{390} & Rhytideres sexsulcatus (Heer, 1856) & Aix & P3h & 146 \\
\hline & $=$ Phytonomus annosus Oustalet, 1874 & & & 110 \\
\hline & =Hipporhinus reynesii Oustalet, 1874 & & & 110 \\
\hline \multirow[t]{6}{*}{391} & "Rh." heeri (Germar, 1849) & & P3h & 147 \\
\hline & =Hipporhinus Matheroni Nicolas, 1891 & & & 148 \\
\hline & $=$ Hipporhinus similis Nicolas, 1891 & & & 148 \\
\hline & $=$ Hipporhinus intermedius Nicolas, 1891 & Aix & & 148 \\
\hline & =Hipporhinus Marioni Nicolas, 1891 & Aix & & 148 \\
\hline & $=$ Hipporhinus pertonii Nicolas, 1891 & & & 148 \\
\hline 392 & "Rh." schaumi (Heer, 1856) & & P3h & 146 \\
\hline 393 & "Hipporhinus" brevis Giebel, 1856 & & P3h & 149 \\
\hline \multirow{2}{*}{\multicolumn{5}{|c|}{$\begin{array}{l}\text { Entimini Schoenherr, } 1823 \\
\text { Entimina Schoenherr, } 1823\end{array}$}} \\
\hline & & & & \\
\hline 394 & Entimus primordialis Scudder, 1876 & GreR & P2i-1 & 73 \\
\hline
\end{tabular}


Table 2. Cont.

\begin{tabular}{|c|c|c|c|c|}
\hline No. & Taxon & Locality & Age & Sources \\
\hline \multicolumn{5}{|c|}{ Eudiagogini LeConte, 1874} \\
\hline 395 & Eudiagogus vossi Legalov, 2018 & GreR & P2i-1 & 69 \\
\hline 396 & Tolstonosik oisensis Legalov, Kirejtshuk et Nel, 2019 & OisJ & $\mathrm{P} 2 \mathrm{i}$ & 81 \\
\hline 397 & Oligocryptus sectus (Scudder, 1893) & Flor & $\mathrm{P} 2 \mathrm{p}$ & 77 \\
\hline 398 & Eudomus pinguis Scudder, 1893 & Flor & P2p & 77 \\
\hline 399 & E. robustus Scudder, 1893 & Flor & $\mathrm{P} 2 \mathrm{p}$ & 77 \\
\hline \multicolumn{5}{|c|}{ Hyperitae Lacordaire, 1863} \\
\hline \multicolumn{5}{|c|}{ Hyperini Lacordaire, 1863} \\
\hline \multicolumn{5}{|c|}{ Cepurina Capiomont, 1867} \\
\hline 400 & Palaeophelypera kuscheli Legalov, 2013 & BalJ & $\mathrm{P} 2 \mathrm{~b}$ & 47 \\
\hline 401 & "Geralophus" discessus Scudder, 1893 & Flor & $\mathrm{P} 2 \mathrm{p}$ & 77 \\
\hline 402 & Hyperites nadezhkini Zherikhin, 1989 & Biam & $\mathrm{P} 2 \mathrm{p}$ & 150 \\
\hline \multirow{2}{*}{\multicolumn{5}{|c|}{$\begin{array}{l}\text { Otiorhynchitae Schoenherr, } 1826 \\
\text { Hormorini Horn, } 1876\end{array}$}} \\
\hline & & & & \\
\hline 403 & "Hormorus" saxorum Scudder, 1893 & Flor & P2p & 77 \\
\hline \multirow{2}{*}{\multicolumn{5}{|c|}{$\begin{array}{c}\text { Cyphiceritae Lacordaire, } 1863 \\
\text { Sciaphilini Sharp, } 1891\end{array}$}} \\
\hline & & & & \\
\hline 404 & “Mitostylus" obdurefactus (Scudder, 1893) & RoaM & P2i-1 & 77 \\
\hline 405 & "M." seculorum (Scudder, 1890) & GreR & P2i-1 & 78 \\
\hline 406 & "M." abacus (Scudder, 1893) & WhiR & $\mathrm{P} 3 \mathrm{r}$ & 77 \\
\hline \multirow{2}{*}{\multicolumn{5}{|c|}{$\begin{array}{l}\text { Trachyphloeini Lacordaire, } 1863 \\
\text { Pseudocneorrhinina Kono, } 1930\end{array}$}} \\
\hline & & & & \\
\hline 407 & Archaeocallirhopalus alekseevi Legalov et Bukejs, 2015 & BalJ & $\mathrm{P} 2 \mathrm{~b}$ & 151 \\
\hline 408 & A. larssoni Legalov, 2013 & BalJ & $\mathrm{P} 2 \mathrm{~b}$ & 47 \\
\hline \multirow{2}{*}{\multicolumn{5}{|c|}{$\begin{array}{l}\text { Polydrusitae Schoenherr, } 1823 \\
\text { Sitonini Gistel, } 1856\end{array}$}} \\
\hline & & & & \\
\hline 409 & Sitonitellus egregius (Haupt, 1956) & Geis & P21 & 152 \\
\hline 410 & Sitona lata Theobald, 1937 & Klei & P3 & 112 \\
\hline 411 & “S." venustulus Heyden et Heyden, 1866 & Rott & P3h & 85 \\
\hline \multicolumn{5}{|c|}{ Anypotactini Champion, 1911} \\
\hline 412 & $\begin{array}{l}\text { Paonaupactus gracilis Legalov, Nazarenko et Perkovsky, } \\
2019\end{array}$ & RovJ & $\mathrm{P} 2 \mathrm{~b}$ & 117 \\
\hline 413 & P. katyae Legalov, Nazarenko and Perkovsky, 2019 & RovJ & $\mathrm{P} 2 \mathrm{~b}$ & 117 \\
\hline 414 & P. microphthalmus (Zherikhin, 1971) & BalJ & $\mathrm{P} 2 \mathrm{~b}$ & 79 \\
\hline \multirow[t]{4}{*}{415} & P. sitonitoides Voss, 1953 & BalJ, & $\mathrm{P} 2 \mathrm{~b}$ & 80 \\
\hline & $=$ Polydrosus scheelei Voss, 1953 & ScanJ, & & 80 \\
\hline & =Pyllobius cephalotes Voss, 1972 & PolJ & & 105 \\
\hline & =Otiorhynchus pellucidipes Voss, 1972 & & & 105 \\
\hline 416 & P. sobrinus (Voss, 1972) & $\begin{array}{l}\text { ScanJ, } \\
\text { Balj }\end{array}$ & $\mathrm{P} 2 \mathrm{~b}$ & 105 \\
\hline 417 & P. viridis (Wanat et Borowiec, 1986) & BalJ, PolJ & $\mathrm{P} 2 \mathrm{~b}$ & 153 \\
\hline \multicolumn{5}{|c|}{ Naupactini Gistel, 1856} \\
\hline 418 & “Hipporhinus" ventricosus (Piton, 1940) & Mena & P1sl-t & 99 \\
\hline 419 & Arostropsis groehni Yunakov et Kirejtshuk, 2011 & BalJ & $\mathrm{P} 2 \mathrm{~b}$ & 154 \\
\hline 420 & A. gusakovi Legalov, 2020 & BalJ & $\mathrm{P} 2 \mathrm{~b}$ & 71 \\
\hline 421 & A. perkovskyi Bukejs et Legalov, 2019 & RovJ & $\mathrm{P} 2 \mathrm{~b}$ & 155 \\
\hline 422 & "Cyrtomon" subterraneus Wickham, 1911 & Flor & $\mathrm{P} 2 \mathrm{p}$ & 76 \\
\hline 423 & "C." florissantensis Wickham, 1914 & Flor & $\mathrm{P} 2 \mathrm{p}$ & 156 \\
\hline
\end{tabular}


Table 2. Cont.

\begin{tabular}{|c|c|c|c|c|}
\hline No. & Taxon & Locality & Age & Sources \\
\hline \multicolumn{5}{|c|}{ Geonemini Gistel, 1856} \\
\hline 424 & “Epicaerus" dilapsus (Scudder, 1893) & GreR & P2i-1 & 77 \\
\hline 425 & “E." effossus (Scudder, 1876) & GreR & P2i-1 & 73 \\
\hline 426 & "E." eradicatus (Scudder, 1893) & WhiR & P2i-1 & 77 \\
\hline 427 & "E." exanimis (Scudder, 1876) & GreR & P2i-1 & 73 \\
\hline 428 & “E." excissus (Scudder, 1893) & RoaM & P2i-1 & 77 \\
\hline 429 & "E." fodinarum (Scudder, 1893) & GreR & P2i-1 & 77 \\
\hline 430 & "E." saxatilis (Scudder, 1876) & GreR & P2i-1 & 73 \\
\hline 431 & "E." subterraneus (Scudder, 1893) & GreR & P2i-1 & 77 \\
\hline 432 & “E.” terrosus (Scudder, 1878) & RoaM, WhiR & $\begin{array}{l}\text { P2i-1, } \\
\text { P3r }\end{array}$ & 74 \\
\hline 433 & "E." evigoratus (Scudder, 1893) & WhiR & $\mathrm{P} 3 \mathrm{r}$ & 77 \\
\hline 434 & Evopes veneratus Scudder, 1893 & Flor & P2p & 77 \\
\hline 435 & E. occubatus Scudder, 1893 & Flor & $\mathrm{P} 2 \mathrm{p}$ & 77 \\
\hline 436 & "Lachnopus" recuperatus Scudder, 1893 & Flor & $\mathrm{P} 2 \mathrm{p}$ & 77 \\
\hline 437 & “Omileus” evanidus Scudder, 1893 & Flor & P2p & 77 \\
\hline \multicolumn{5}{|c|}{ Psallidiini Lacordaire, 1863} \\
\hline 438 & Trigonoscuta inventa Scudder, 1893 & Flor & $\mathrm{P} 2 \mathrm{p}$ & 77 \\
\hline \multicolumn{5}{|c|}{ Eustylini Lacordaire, 1863} \\
\hline 439 & Pseudophaops perditus (Scudder, 1876) & GreR & P2i-1 & 73 \\
\hline \multicolumn{5}{|c|}{ Polydrusini Schoenherr, 1823} \\
\hline 440 & Polydrusus archetypus Zherikhin, 1971 & BalJ & $\mathrm{P} 2 \mathrm{~b}$ & 79 \\
\hline 441 & P. zherikhini Legalov, 2020 & BalJ & $\mathrm{P} 2 \mathrm{~b}$ & 71 \\
\hline 442 & Archaeosciaphilus Marshalli Legalov, 2012 & BalJ & $\mathrm{P} 2 \mathrm{~b}$ & 82 \\
\hline \multicolumn{5}{|c|}{ Brachyderini Schoenherr, 1826} \\
\hline 443 & Palaeocrassirhinus messelensis Rheinheimer, 2007 & Mess & P2l & 102 \\
\hline 444 & P. rugosithorax Rheinheimer, 2007 & Mess & P21 & 102 \\
\hline 445 & Palaeocneorhinus messelensis Rheinheimer, 2007 & Mess & P21 & 102 \\
\hline 446 & "Brachyderes" rugosus (Deichmueller, 1881) & Kuts & $\mathrm{P} 2 \mathrm{~b}$ & 126 \\
\hline 447 & Brachymycterus curculionoides Heyden et Heyden, 1866 & Rott & P3h & 85 \\
\hline & $=$ Eurychirus induratus Heyden et Heyden, 1866 & & & 85 \\
\hline & =Rhinocyllus improbus Heyden et Heyden, 1866 & & & 85 \\
\hline & $=$ Varus ignotus Schlechtendal, 1894 & & & 89 \\
\hline \multicolumn{5}{|c|}{$\begin{array}{c}\text { Tanymecini Lacordaire, } 1863 \\
\text { Tainophthalmina Desbrochers des Loges, } 1873\end{array}$} \\
\hline 448 & Protainophthalmus asperulus (Heer, 1856) & Aix & P3h & 146 \\
\hline & $=$ Brachyderes aquisextanus Oustalet, 1874 & & & 110 \\
\hline & $=$ Brachyderes longipes Oustalet, 1874 & & & 110 \\
\hline & $=$ Cleonus Marcelli Oustalet, 1874 & & & 110 \\
\hline 449 & P. margarum (Germar, 1849) & Aix & P3h & 147 \\
\hline & =Sitona antiqua Giebel, 1856 & & & 149 \\
\hline 450 & P. punctulatus (Nicolas, 1891) & Aix & P3h & 148 \\
\hline 451 & P. regularis (Nicolas, 1891) & Aix & P3h & 148 \\
\hline 452 & P. thaisi (Nicolas, 1891) & Aix & P3h & 148 \\
\hline 453 & P. tuberculatus (Nicolas, 1891) & Aix & P3h & 148 \\
\hline \multicolumn{5}{|c|}{ Pandeleteina Pierce, 1913} \\
\hline 454 & Pandeleteinus nudus Wickham, 1917 & Flor & $\mathrm{P} 2 \mathrm{p}$ & 138 \\
\hline & Tribe incertae sedis & & & \\
\hline
\end{tabular}


Table 2. Cont.

\begin{tabular}{|c|c|c|c|c|}
\hline No. & Taxon & Locality & Age & Sources \\
\hline 455 & "Sitona" exitiorum Scudder, 1893 & Flor & $\mathrm{P} 3 \mathrm{r}$ & 77 \\
\hline 456 & "Strophosomus" marcelini Theobald, 1937 & Cela & $\mathrm{P} 2 \mathrm{p}$ & 112 \\
\hline 457 & "Sciaphilus" nigrescens Theobald, 1937 & Cela & $\mathrm{P} 2 \mathrm{p}$ & 112 \\
\hline 458 & "?Argoptochus" incertus Theobard, 1937 & Cela & $\mathrm{P} 2 \mathrm{p}$ & 112 \\
\hline 459 & "Bagous" atavus Oustalet, 1870 & Core & $\mathrm{P} 3 \mathrm{r}$ & 119 \\
\hline 460 & "Brachycerus" exilis Germar, 1837 & Aix & P3h & 157 \\
\hline 461 & "Phytonomus" firmus Heer, 1856 & Aix & P3h & 146 \\
\hline \multicolumn{5}{|c|}{ Curculionidae incertae sedis } \\
\hline 462 & Otiorhynchites williamsi Cockerell, 1943 & Mirs & P1 & 158 \\
\hline 463 & O. aterrimus Cockerell, 1925 & Sunc & P1d & 134 \\
\hline 464 & O. crassus Cockerell et Wagner, 1936 & Sunc & P1d & 159 \\
\hline 465 & O. densepunctatus Haupt, 1956 & Geis & $\mathrm{P} 21$ & 152 \\
\hline 466 & O. fossilis Scudder, 1893 & GreR & P2i-1 & 77 \\
\hline 467 & O. commutatus Scudder, 1893 & RoaM & P2i-1 & 77 \\
\hline 468 & O. tysoni Scudder, 1893 & RoaM & P2i-1 & 77 \\
\hline 469 & O. absentivus Scudder, 1893 & Flor & $\mathrm{P} 2 \mathrm{p}$ & 77 \\
\hline 470 & O. florissantensis Wickham, 1911 & Flor & $\mathrm{P} 2 \mathrm{p}$ & 76 \\
\hline 471 & O. wilcoxianus Wickham, 1929 & Flor & $\mathrm{P} 2 \mathrm{p}$ & 160 \\
\hline 472 & Curculionites taxodii Heer, 1870 & Star & $\mathrm{P} 1 \mathrm{~d}$ & 161 \\
\hline 473 & C. angustior Cockerell et Wagner, 1936 & Sunc & P1d & 159 \\
\hline 474 & C. epistictus Cockerell et Wagner, 1936 & Sunc & P1d & 159 \\
\hline 475 & C. eustictus Cockerell et Wagner, 1936 & Sunc & P1d & 159 \\
\hline 476 & C. harringtoni Cockerell, 1925 & Sunc & P1d & 134 \\
\hline 477 & C. jujuensis Cockerell, 1925 & Sunc & P1d & 134 \\
\hline 478 & C. latiusculus Cockerell et Wagner, 1936 & Sunc & P1d & 159 \\
\hline 479 & C. magdalinus Cockerell et Wagner, 1936 & Sunc & P1d & 159 \\
\hline 480 & C. megastictus Cockerell et Wagner, 1936 & Sunc & P1d & 159 \\
\hline 481 & C. microstictus Cockerell et Wagner, 1936 & Sunc & P1d & 159 \\
\hline 482 & C. parastictus Cockerell et Wagner, 1936 & Sunc & P1d & 159 \\
\hline 483 & C. stebingeri Cockerell, 1926 & Sunc & P1d & 131 \\
\hline 484 & C. sunchalicus Cockerell et Wagner, 1936 & Sunc & P1d & 159 \\
\hline 485 & C. wielandi Cockerell, 1925 & Sunc & P1d & 134 \\
\hline 486 & C. marginatus Giebel, 1856 & Corf & P2l & 149 \\
\hline 487 & C. punctillatus Haupt, 1950 & Geis & P2l & 124 \\
\hline 488 & C. bartonicus Cockerell, 1920 & Bour & P21 & 162 \\
\hline 489 & C. brenthiformis Cockerell, 1920 & Bour & P21 & 162 \\
\hline 490 & C. optimus Cockerell, 1920 & Bour & P2l & 162 \\
\hline 491 & C. ovatus Oustalet, 1870 & Core & P3r & 119 \\
\hline 492 & C. morosus Heer, 1856 & Aix & P3h & 146 \\
\hline 493 & Calandrites defessus Scudder, 1893 & RoaM & P2i-1 & 77 \\
\hline 494 & C. cineratius Scudder, 1893 & GreR,RoaM & P2i-1 & 77 \\
\hline 495 & C. hindsi Cockerell, 1917 & Flor & $\mathrm{P} 2 \mathrm{p}$ & \\
\hline 496 & C. ursorum Cockerell, 1918 & Flor & $\mathrm{P} 2 \mathrm{p}$ & \\
\hline 497 & Ophryastites gardneri Cockerell, 1920 & Bour & P21 & \\
\hline 498 & O. absconsus Scudder, 1893 & Flor & $\mathrm{P} 2 \mathrm{p}$ & 77 \\
\hline 499 & O. cinereus Scudder, 1893 & Flor & $\mathrm{P} 2 \mathrm{p}$ & 77 \\
\hline 500 & O. digressus Scudder, 1893 & Flor & $\mathrm{P} 2 \mathrm{p}$ & 77 \\
\hline 501 & O. hendersoni Cockerell, 1917 & Flor & $\mathrm{P} 2 \mathrm{p}$ & \\
\hline 502 & O. miocenus Wickham, 1912 & Flor & $\mathrm{P} 2 \mathrm{p}$ & \\
\hline 503 & O. dispertitus Scudder, 1893 & WhiR & $\mathrm{P} 3 \mathrm{r}$ & 77 \\
\hline 504 & O. heribaudi Piton, 1936 & PuStJ & P3 & \\
\hline 505 & Mononychites punctipennis Haupt, 1956 & Geis & P2l & \\
\hline 506 & M. rotundatus Haupt, 1956 & Geis & P2l & \\
\hline 507 & Syntomostylus rudis Scudder, 1893 & RoaM & P2i-1 & \\
\hline 508 & "S." fortis Cockerell, 1909 & GreR & P2i-1 & \\
\hline 509 & "Otiorhynchus" flaccus Scudder, 1893 & RoaM & P2i-1 & 77 \\
\hline
\end{tabular}


Table 2. Cont.

\begin{tabular}{|c|c|c|c|c|}
\hline No. & Taxon & Locality & Age & Sources \\
\hline 510 & "Otiorhynchus" tumbae Scudder, 1890 & GreR & P2i-1 & \\
\hline 511 & "Pachylobius" depraedatus Scudder, 1893 & RoaM & P2i-1 & 77 \\
\hline 512 & "Pachylobius" compressus Scudder, 1893 & GreR,RoaM & P2i-1 & 77 \\
\hline 513 & "Phyllobius" antecessor Scudder, 1893 & RoaM & P2i-1 & 77 \\
\hline 514 & "Rhyssomatus" tabescens Scudder, 1893 & WhiR & $\mathrm{P} 3 \mathrm{r}$ & 77 \\
\hline 515 & "Scythropus" somniculosus Scudder, 1893 & WhiR & $\mathrm{P} 3 \mathrm{r}$ & 77 \\
\hline 516 & "Phyllobius" avus Scudder, 1893 & RoaM & P2i-1 & 77 \\
\hline 517 & "Phyllobius" carcerarius Scudder, 1893 & RoaM & P2i-1 & 77 \\
\hline 518 & “Pachylobius" yungi Piton, 1936 & Gaub & P3 & 164 \\
\hline 519 & "Tanymecus" gautieri Piton, 1936 & Gaub & P3 & 164 \\
\hline 520 & "Pachylobius" martyi Piton et Theobald, 1937 & PuStJ & P3 & 166 \\
\hline 521 & "Lachnopus" dilatatus Theobald, 1937 & Klei & P3 & 166 \\
\hline 522 & “Cleonus” foersteri Theobald, 1937 & Klei & P3 & 112 \\
\hline 523 & "Lachnopus" humatus Scudder, 1893 & Flor & $\mathrm{P} 2 \mathrm{p}$ & 77 \\
\hline 524 & "Sphenophorus" elegans Theobald, 1935 & Aix & P3h & 130 \\
\hline \multirow{3}{*}{\multicolumn{5}{|c|}{$\begin{array}{l}\text { Platypodidae Shuckard, } 1840 \\
\text { Tesserocerinae Strohmeyer, } 1914 \\
\text { Tesserocerini Strohmeyer, } 1914\end{array}$}} \\
\hline & & & & \\
\hline & & & & \\
\hline 525 & $\begin{array}{l}\text { Eoplatypus jordali Peris, Solórzano Kraemer et Cognato, } \\
2017\end{array}$ & BalJ & $\mathrm{P} 2 \mathrm{~b}$ & 167 \\
\hline 526 & Cenocephalus aniskini Legalov, 2020 & BalJ & $\mathrm{P} 2 \mathrm{~b}$ & 71 \\
\hline \multirow{3}{*}{\multicolumn{5}{|c|}{$\begin{array}{c}\text { Scolytidae Latreille, } 1804 \\
\text { Hylesininae Erichson, } 1836 \\
\text { HylastiniLeConte, } 1876\end{array}$}} \\
\hline & & & & \\
\hline & & & & \\
\hline 527 & Hylastes aterites Schedl, 1947 & BalJ & $\mathrm{P} 2 \mathrm{~b}$ & 168 \\
\hline 528 & "H." americanus Wickham, 1913 & Flor & $\mathrm{P} 2 \mathrm{~b}$ & 97 \\
\hline 529 & Hylurgops corpulentus Schedl, 1947 & BalJ & $\mathrm{P} 2 \mathrm{~b}$ & 168 \\
\hline 530 & H. dubius (Hagedorn, 1906) & BalJ & $\mathrm{P} 2 \mathrm{~b}$ & 169 \\
\hline 531 & H. electrinus (Germar, 1813) & BalJ & $\mathrm{P} 2 \mathrm{~b}$ & 170 \\
\hline 532 & H. granulatus (Schedl, 1947) & BalJ & $\mathrm{P} 2 \mathrm{~b}$ & 168 \\
\hline 533 & H. pilosellus Schedl, 1947 & BalJ & $\mathrm{P} 2 \mathrm{~b}$ & 168 \\
\hline 534 & H. schellwieni (Hagedorn, 1906) & BalJ & $\mathrm{P} 2 \mathrm{~b}$ & 168 \\
\hline 535 & H. tuberculatus Schedl, 1947 & BalJ & $\mathrm{P} 2 \mathrm{~b}$ & 168 \\
\hline 536 & H. piger Wickham, 1913 & Flor & $\mathrm{P} 2 \mathrm{p}$ & 97 \\
\hline \multicolumn{5}{|c|}{ HylesininiErichson, 1836} \\
\hline 537 & Hylesinus extractus Scudder, 1893 & Flor & $\mathrm{P} 2 \mathrm{p}$ & 77 \\
\hline 538 & H. hydropicus (Wickham, 1916) & Flor & $\mathrm{P} 2 \mathrm{p}$ & 101 \\
\hline 539 & H. neli Petrov et Zherikhin, 2004 & Aix & P3h & 171 \\
\hline 540 & “H.” facilis Heer, 1856 & Aix & P3h & 146 \\
\hline \multicolumn{5}{|c|}{ Hylurgini Gistel, 1848} \\
\hline 541 & Xylechinus mozolevskae Petrov et Perkovsky, 2008 & RovJ & $\mathrm{P} 2 \mathrm{~b}$ & 172 \\
\hline 542 & Klesovia pubescens Petrov et Perkovsky, 2018 & RovJ & $\mathrm{P} 2 \mathrm{~b}$ & 173 \\
\hline 543 & Xylechinites anceps Hagedorn, 1906 & BalJ & $\mathrm{P} 2 \mathrm{~b}$ & 169 \\
\hline \multicolumn{5}{|c|}{ Phloeosinini Nuesslin, 1912} \\
\hline 544 & Phloeosinus assimilis (Schedl, 1947) & BalJ & $\mathrm{P} 2 \mathrm{~b}$ & 168 \\
\hline 545 & Ph. brunni (Hagedorn, 1906) & BalJ & $\mathrm{P} 2 \mathrm{~b}$ & 169 \\
\hline 546 & Ph. regimontanus (Hagedorn, 1906) & BalJ & $\mathrm{P} 2 \mathrm{~b}$ & 169 \\
\hline 547 & Ph. rehi (Hagedorn, 1906) & BalJ & $\mathrm{P} 2 \mathrm{~b}$ & 169 \\
\hline 548 & Ph. robustus (Schedl, 1947) & BalJ & $\mathrm{P} 2 \mathrm{~b}$ & 168 \\
\hline 549 & Ph. sexspinosus (Schedl, 1947) & BalJ & $\mathrm{P} 2 \mathrm{~b}$ & 168 \\
\hline 550 & Ph. tuberculifer (Schedl, 1947) & BalJ & $\mathrm{P} 2 \mathrm{~b}$ & 168 \\
\hline 551 & Ph. wolffi (Schedl, 1947) & BalJ & $\mathrm{P} 2 \mathrm{~b}$ & 168 \\
\hline
\end{tabular}


Table 2. Cont.

\begin{tabular}{|c|c|c|c|c|}
\hline No. & Taxon & Locality & Age & Sources \\
\hline \multicolumn{5}{|c|}{ Phloeotribini Chapuis, 1869} \\
\hline 552 & Phloeotribus zimmermani Wickham, 1916 & Flor & $\mathrm{P} 2 \mathrm{p}$ & 101 \\
\hline \multicolumn{5}{|c|}{ Polygraphini Chapuis, 1869} \\
\hline 553 & Carphoborus keilbachi (Schedl, 1947) & BalJ & $\mathrm{P} 2 \mathrm{~b}$ & 168 \\
\hline 554 & C. posticus (Schedl, 1947) & BalJ & $\mathrm{P} 2 \mathrm{~b}$ & 168 \\
\hline \multicolumn{5}{|c|}{$\begin{array}{c}\text { Scolytinae Latreille, } 1804 \\
\text { DryocoetiniLindemann, } 1877\end{array}$} \\
\hline 555 & Dryocoetes diluvialis (Wickham, 1916) & GreR BalJ & $\begin{array}{l}\mathrm{P} 2 \mathrm{i}-1 \\
\mathrm{P} 2 \mathrm{~b}\end{array}$ & 101 \\
\hline 556 & Taphramites gnathotrichus Schedl, 1947 & RovJ & $\mathrm{P} 2 \mathrm{~b}$ & 168 \\
\hline 557 & T. rovnoensis Petrov et Perkovsky, 2008 & BalJ & $\mathrm{P} 2 \mathrm{~b}$ & 172 \\
\hline 558 & Taphrorychus immaturatus Schedl, 1947 & & & 168 \\
\hline \multicolumn{5}{|c|}{ Incertae sedis } \\
\hline 559 & "Dryocoetes" carbonarius Scudder, 1878 & GreR & P2i-1 & 74 \\
\hline 560 & “Trypodendron" impressus Scudder, 1876 & GreR & P2i-1 & 73 \\
\hline 561 & "Polygraphus" wortheni Scudder, 1893 & RoaM & P2i-1 & 77 \\
\hline 562 & Xyleborites longipennis Wickham, 1913 & Flor & $\mathrm{P} 2 \mathrm{p}$ & 97 \\
\hline 563 & Duartia pulchella Martins-Neto, 2001 & Fons & $\mathrm{P} 2 \mathrm{p}$ & 174 \\
\hline \multicolumn{5}{|c|}{ Curculionoidea incertae sedis } \\
\hline 564 & Thryogenosoma cariniger (Motschulsky, 1857) & BalJ & $\mathrm{P} 2 \mathrm{~b}$ & 175 \\
\hline
\end{tabular}

\subsection{Paleocene Weevil Fauna}

The weevil fauna of the Paleocene is the poorest among the Paleogene faunas. This is primarily due to the small number of Paleocene localities, as well as the lack of Paleocene amber with Curculionoidea. Curculionoid beetles were found in five localities, including France, Svalbard (Denmark), south of the Russian Far East, China (Hong Kong) and Argentina (Figure 2). Three of these localities are of early Paleocene age (Arkhara, Starostin and Sunchal) and one of them is late Paleocene (Menat). The age of Mirs Bay from Hong Kong, the Ping Chau Formation (China) is assumed to be Paleocene, however the stage is not specified [176].

Twenty seven species were described from the Paleocene (Figure 3). Additionally, 18 species were known from Starostin, Sunchal and Mirs based on isolated elytra, which are assigned to recent genera or placed in the formal genera Otiorhynchites and Curculionites that were established for elytra $[131,134,158,159,161]$. "Cossonus" devoratus and "Anthonomus" sunchalensis from the early Paleocene of Argentina are not only the earliest findings of the tribes Cossonini and Anthinomini but also the first appearance of the subfamilies Cossoninae and Curculioninae in the fossil record. The only early Paleocene beetle represented by an almost complete impression is Archaralites zherichini of the subfamily Molytinae (earliest record of this subfamily) from the Danian of Outer Manchuria.

Eight species represented as complete impressions are known from Selandian-Thanetian of France. The families Ithyceridae, Rhynchitidae, Attelabidae, Brentidae and Curculionidae are recorded. Ithyceridae is represented by one species of the subfamily Chilecarinae. This is the only representative of the family in the Paleocene and the last find in the Eastern Hemisphere. Records of Ithyceridae are common in Cretaceous of the Northern Hemisphere [29,30,48,49,51,177]. One species, the genus of which requires clarification, is from the subfamily Rhynchitinae of the family Rhynchitidae. The families Attelabidae and Brentidae are each represented by one species of the recent tribe Attelabini and the extinct species of the recent genus Perapion from the tribe Aplemonini (earliest record, 61.0-59.0 Ma). 
Four species belong to the family Curculionidae. One species of the recent genus Lixus is from the subfamily Lixinae (earliest record, 61.0-59.0 Ma). Two species of the subfamily Curculioninae, one of which belongs to the extinct genus of the tribe Curculionini (earliest record, 61.0-59.0 Ma), and the systematic position of the second ("Centrinus" longipes) in the subfamily require clarification. One species, "Hipporhinus" ventricosus with an unclear generic position, is in the tribe Naupactini from the diverse subfamily Entiminae (earliest record, 61.0-59.0 Ma). Representatives of other families were not found in the Paleocene.

Most of the forms represented in the Paleocene belong to widespread groups (Attelabini, Aplemonini (Perapion), Lixini (Lixus), Cossonini (Cossonus), Curculionini, Anthonomini (Anthonomus)), are now found in the recent fauna. Two species show characters typical of the Paleocene fauna of Europe. Petropsis rostratus somehow links the fauna of Menat with recent relict fauna of the Chilean-Patagonian and Australian regions, where modern representatives of the tribe Chilecarini live $[178,179]$. The discovery of species of the tribe Naupactini similar to Neotropical forms provides additional evidence for the faunogenetic relationships of the Paleocene Europe, with Central and South America. The ecological composition of these beetles is homogeneous. Mostly they are forest forms. Petropsis rostratus, like the recent Ithyceridae of the subfamily Chilecarinae, could be associated with gymnosperms from the family Cupressaceae [178,179], which were found at the site [99]. "Phytonomus" punctatus (Attelabini) folded tubes from angiosperm leaves, for example, from oak known from the deposit [99], as recent representatives of this tribe [180]. Archaralites zherichini and "Cossonus" devoratus developed in wood. Menatorhis elegans, like modern Curculionini, developed in flower buds or fruits of angiosperms [181], for example, on oak, several species that are known from this locality [99]. "Anthonomus" sunchalensis could be connected with trees or shrubs. Two species were very probably associated with herbaceous vegetation, the recent species of the genus Lixus usually develop on herbaceous plants and, as an exception, with shrubby plants [182]. Lixus ligniticus could be associated with Atriplex (Amaranthaceae). Perapion menatensis, as well as recent representatives of the genus, probably developed on Polygonaceae [183]. Polygonaceae are not known from Menat but pollen of Polygonum was recorded in the late Paleocene of France [184]. Curculionoidea of Menat shows the presence of coniferous-deciduous forest and herbaceous near-water vegetation.

\subsection{Eocene Weevil Fauna}

As Many as 441 species of weevil-shaped beetles were described from Eocene deposits (Figure 4), which originated from 24 localities. Undescribed forms are known from Quilchena (Canada) [185], Romanian amber [186], Huitrera Formation (Argentina) [187] and Bitterfeld amber.

\subsubsection{Review of Curculionoidea Families in the Eocene}

Nemonychidae in the Eocene

Three species of the family Nemonychidae from the extinct subfamily Cretonemonychinae and recent Cimberidinae were found in the Eocene. The subfamily Cretonemonychinae is also known in the early and late Cretaceous $[29,30,177]$.

\section{Anthribidae in the Eocene}

Nearly thirty species of Anthribidae were described from the early, middle and late Eocene of America and Europe. The subfamily Anthribinae is most represented. Twenty seven species from eight tribes are known in this subfamily from the Eocene. Twelve species from three genera of the tribes Mecocerini, Allandrini, Oiserhinini and Zygaenodini were described from European amber and 15 species from seven genera of the tribes Cratoparini, Anthribini, Ecelonerini and Tropiderini from North American localities. There are no common genera and tribes between the American and European deposits. The subfamily Choraginae is represented by two species from two tribes. "Choragus" fictilis 
of the tribe Choragini was described from the Eocene of the United States and Eduardoxenus unicus of the tribe Valenfriesiini from Rovno amber.

Ithyceridae in the Eocene

Two American genera Eoceneithycerus and Ithyceroides close to the recent American genus Ithycerus were found in North America.

\section{Belidae in the Eocene}

Nine species from the subfamily Oxycoryninae belong to the family Belidae. Several species of the tribe Oxycraspedini were found in Baltic amber, one of which was described. The tribe Metrioxenini is found both in American deposits and the middle Eocene amber. The extinct tribe Palaeorhopalotriini, close to the recent Central American tribe Allocorynin, was found in the Eocene of France.

\section{Rhynchitidae in the Eocene}

The family Rhynchitidae is found in American and European deposits and is represented by 34 described species. Five species of the genus Baltocar of the tribe Sanyrevilleini from the subfamily Sayrevilleinae are known only from Baltic amber. The subfamily Rhynchitinae is represented by species of the tribes Auletini, Rhynchitini and Eugnamptini. Thirteen species from subtribes Auletina, Pseudauletina and Pseudomesauletina were described in the tribe Auletini. Most species (nine) are known from the terminal Eocene of Florissant. Finds of Auletini are rare in the Green River deposits as well as in Baltic and Rovno amber. The tribe Rhynchitini is represented by 12 species, with nine of them recorded in the late Eocene of the United States and their affiliation to the subtribes has not yet been established. Two species from Baltic amber belong to the archaic subtribes Temnocerina and Perrhynchitina. One genus with three species of the tribe Eugnamptini is described from the Florissant beds.

\section{Attelabidae in the Eocene}

One or two extinct genera, Palaeoalatorostrum of the subtribe Attelabini from the tribe Attelabini and Paleoclinolabus of the subtribe Clinolabina from the tribe Euscelini, belonging to the subfamily Attelabinae of the Attelabidae, are known from the middle Eocene of Germany and the Late Eocene of the United States.

\section{Brentidae in the Eocene}

The family Brentidae is represented in the Eocene by three subfamilies, the Apioninae, Nanophyinae and Brentinae. Thirty six species belong to this family. The subfamily Apioninae is the most species-rich. The primitive tribes Tanaini, Rhadinocybini, Notapionini and Palaeotanaini each have one monotypic genus in the early Eocene of Europe and Asia, as well as in the end of the middle Eocene of Europe. The supertribes Aspidapiitae and Apionitae include representatives of recent (Pseudaspidapion, Melanapion, Conapium, Perapion, Apionion and Toxorhynchus) and extinct genera (Baltoapion, Succinapion, Baltoconapium, Electrapion). A third of the species of the latter groups belong to the extinct genera and two-thirds to the recent ones. Both known tribes (Nanophyini and Corimaliini) of the subfamily Nanophyinae were found in the Eocene. Three extinct genera of the Nanophyini were described from the middle Eocene of the USA and Baltic amber. One species placed in the genus Corimalia is known from the middle Eocene of France. The subfamily Brentinae is recorded only in the middle Eocene of Germany, where the extinct genus Eckfelderolispa with three species and extinct representative of the recent genus Cerobates, belonging to the tribe Trachelizini, were described. 
Curculionidae in the Eocene

The Curculionidae is the Main group of Eocene Curculionoidea numbering 290 described species. All known subfamilies (Erirhininae, Molytinae, Lixinae, Dryophthorinae, Cossoninae, Conoderinae, Curculioninae, Cyclominae and Entiminae) are represented in the Eocene (Figure 5).

The most primitive subfamily Erirhininae is represented by species from the tribes Erirhinini and Dorytomini. Several species from different genera of the former tribe were recorded in the early Eocene of England, the middle and the late Eocene of the USA. The genus Dorytomus from the Dorytomini includes nine extinct species from Baltic and Rovno amber, as well as species from the terminal Eocene of North America.

The subfamily Molytinae is one of the most diverse groups of Curculionidae [39,188]. The tribes Molytini, Plinthini, Acicnemidini, Magdalini, Cleogonini, Sciabregmini, Camptorhinini, Aedemonini and Cryptorhynchini were found in Eocene deposits. Two extinct genera, Furhylobius-with one species from the early Eocene of Denmark-and Archaeoheilus—with five species from the early-middle and terminal Eocene of the USA-belong to the tribe Molytini. One species of the recent genus Leiosoma of the tribe Plinthini was described from Baltic amber. One extinct genus Electrotribus of the tribe Acicnemidini is known from Baltic amber, where its species are one of the most common Curculionidae. In other Eocene localities, neither the tribe Acicnemidini, nor this genus were found. "Magdalis" sedimentorum of the tribe Magdalini is recorded from the Eocene of the United States. The American tribe Cleogonini is represented in the Eocene of the United States by two species of the extinct genus Rhysosternum and one species of the genus Conotrachelus. The extinct tribe Sciabregmini with one genus is known from three species, one from the early Eocene of France and two from the early-middle Eocene of North America. Two fossil genera from the early Eocene of England belong to the tribe Camptorhinini. The Afrotropical tribe Aedemonini was recently discovered in Baltic amber. Eleven species from six genera (five of which are extinct) of the tribe Cryptorhynchini are known from the Eocene.

The subfamily Lixinae is represented in the Eocene by only the tribe Cleonini. The extinct genus Eocleonus with one species and five species formally placed in the genus Cleonus were described from the late Eocene of the United States.

Eleven Eocene species belong to the subfamily Dryophthorinae. Most species (seven) are from the tribes Stromboscerini and Dryophthorini living in the forest litter. The former tribe is noted in Baltic and Rovno amber and the latter is in early Eocene Oise amber and late Eocene Florissant deposits. Four species of the tribe Sphenophorini were described from the late Eocene of the United States.

The subfamily Cossoninae is represented by the tribe Dryotribini in middle Eocene amber and Cossonini in early-middle and late Eocene deposits of the United States.

The diverse subfamily Conoderinae is divided into four supertribes [40], three of which are found in Eocene. Thirty species belong to the supertribe Bariditae of which twenty nine were described from early-middle and late Eocene of the USA and one from the middle Eocene of England. Most North American species belong to the subtribe Coelonertina of the tribe Apostasimerini. They were described both in recent (Geraeus, Pachybaris, Nicentrus) and extinct (Miogeraeus, Lithogeraeus, Steganus) genera. Six species belong to the tribe Baridini. These are four representatives of the recent genus Baris and one of the extinct genus Catobaris (Baridina), as well as one species of the recent genus Eurhinus (Eurhinina). Two monotypic extinct genera of the recent tribe Conoderini and the extinct tribe Palaeomallerini are from the early Eocene of France and the early-middle Eocene of the United States belong to the supertribe Conoderintae. The supertribe Ceutorhynchitae is represented by ten species of the tribe Ceutorhynchini and one species of the tribe Cnemogonini, which was noted in the late Eocene of the United States. Two genera, the recent genus Ceutorhynchus Marked from the early to late Eocene of Europe and the USA and extinct Baltocoeliodes from Baltic amber, belong to the tribe Ceutorhynchini.

The subfamily Curculioninae is the second largest group by species of Eocene Curculionidae. Nine tribes with 53 species are known from the Eocene. The tribe Acalyptini is represented by an extinct monotypic genus in early Eocene Oise amber. Two species from Baltic amber belong to the 
tribe Ellescini. The monotypic tribe Palaeoanoplini is known only from the middle Eocene of Europe. Fifteen species were described in the tribe Curculionini. Representatives of the subtribes Erganiina and Timolina are found only in Baltic amber. The most common group in the modern fauna on all continents is the subtribe Curculionina. In the Eocene, all (without Curculio havighorstensis) species of this subtribe were described from the terminal Eocene of the USA and one species from the early Eocene of Germany. The situation is similar with the tribe Anthonomini. All Eocene species of this tribe are known only from North America. The tribe Eugnomini is represented by five species from four genera found in Baltic amber. The extinct subtribe Palaeorhamphina with three species from Baltic amber and the recent subtribe Rhamphina with species of the recent genera Orchestes and Tachyerges from middle Eocene amber and the terminal Eocene of the USA belong to the tribe Rhamphini. The tribe Tychiini is noted in Baltic amber and the late Eocene of the USA, where it is represented by five species from the recent genera Sibinia, Tychius, Macrorhoptus and the extinct genus Eocenesibinia. The tribe Camarotini is noted in the middle Eocene of Europe and the late Eocene of North America.

The subfamily Cyclominae is known only from the Eocene of the USA, where species of the recent genera Listronotus and Listroderes were described. Бщыерlace of the numberrous species among the Eocene Curculionidae are in the subfamily Entiminae. Ninety species were described from the tribes Tropiphorini, Entimini, Eudiagogini, Hyperini, Hormorini, Sciaphilini, Trachyphloeini, Sitonini, Anypotactini, Naupactini, Geonemini, Psallidiini, Eustylini, Polydrusini, Brachyderini and Tanymecini. Twenty four species belong to the tribe Tropiphorini. All but one representative of this tribe were found in the Eocene of North America and only one species of the genus Limalophus has recently been described from middle Eocene Baltic (Polish) amber. The Neotropical tribe Entimini is known from the early-middle Eocene Green River deposits by one extinct species of the recent genus Entimus. The tribe Eudiagogini is now distributed only in the Western Hemisphere [26]. Tolstonosik oisensis was found in the early Eocene of France, Eudiagogus vossi from the early-middle Eocene, Oligocryptus sectus and the genus Eudomus with two species from the late Eocene of the United States. Three species of the tribe Hyperini from the subtribe Cepurina have been described since the end of the middle Eocene of Europe, the late Eocene of the USA and the Far East of Russia. One species, "Hormorus" saxorum Scudder, 1893 from the late Eocene of the USA was described in the American tribe Hormorini. The tribe Sciaphilini with a contemporary centre of diversity in the Western Palaearctic [26] was found only in the early-middle Eocene of North America. The tribe Trachyphloeini is represented by the extinct genus Archaeocallirhopalus with two species belonging to the subtribe Pseudocneorrhinina, which is now distributed in East Asia. One species of the extinct genus Sitonitellus from the middle Eocene of Germany was assigned to the tribe Sitonini. The Neotropical tribe Anypotactini was found in the middle Eocene of Europe. In Baltic amber, this is one of the most common groups. It is interesting to note that this tribe was not found in the Eocene deposits of North America. The tribe Naupactini is noted in Baltic and Rovno amber and in the late Eocene of the Florissant. Thirteen species of the tribe Geonemini are found in the Eocene of the United States. The tribe Psallidiini is represented by one species of the genus Trigonoscuta from the terminal Eocene of North America. The extinct genus Pseudophaops of the tribe Eustylini was described from the early-middle Eocene of Green River. Two species of the genus Polydrusus and one species of the extinct genus Archaeosciaphilus of the tribe Polydrusini were found in Baltic amber. Three species from extinct genera Palaeocrassirhinus and Palaeocneorhinus from the Lutetian of Germany and one species placed in the genus Brachyderes from the Bartonian of the Czech Republic belong to the tribe Brachyderini. Only one species of the tribe Tanymecini was described in the recent genus Pandeleteinus from the late Eocene of North America.

Platypodidae in the Eocene

The family Platypodidae, with two species of the tribe Tesserocerini, were described from Baltic and Romanian amber [71,167,186,189]. 
Scolytidae in the Eocene

Scolytidae is represented by 35 species, 24 of which were described from Baltic and Rovno amber. Twenty six species belong to the subfamily Hylesininae and four to Scolytinae. The systematic position of five species requires clarification. Three genera are extinct, known only from the late Eocene and nine genera are recent. The genera Hylurgops and Phloeosinus are the richest in species with 8 species in each. The largest number of species (21) of bark beetles was described from Baltic amber.

\subsubsection{Early Eocene Weevil Fauna}

Early Eocene (Ypresian) localities (Figure 6) are in the USA, England (Peckham, London Clay), France (Oise amber), Denmark (Mors), Germany (Havighorst) and the south of the Russian Far East (Tadushi). Twenty one species of Curculionoidea were described from these localities (Figure 7). The families Anthribidae, Ithyceridae, Brentidae, Curculionidae and Scolytidae were recorded for Oise amber [190] from the early Eocene. Unfortunately, the fauna of Oise amber and the London clays include only 7-8 described species, with 1-2 species known (Figure 8) from other localities. The USA contains representatives of the subfamily Ithycerinae now living in North America and has a fundamentally different biota from the rest of the Eocene faunas. Common species and genera intermediate between the early Eocene faunas are absent. Representatives of the tribes Oiserhinini, Palaeotanaini, Sciabregmini, Cryptorhynchini, Dryophthorini, Conoderini, Acalyptini, Eudiagogini are found in Oise amber, Ceutorhynchini in Peckham, Curculionini in Havighorst, Erirhinini, Camptorhinini and Cryptorhynchini in London Clay, Molytini in Mors and Tanaini in Tadushi. Here are found some of the earliest records of representatives of the tribes Sciabregmini (53.0 Ma), Cryptorhynchini (54.0-50.0 Ma), Dryophthorini (53.0 Ma), Eudiagogini (53.0 Ma), Ceutorhynchini and Camptorhinini (54.0-50.0 Ma) and the latest find of the tribe Tanaini in the fossil record. The tribes Oiserhinini, Palaeotanaini, Conoderini and Acalyptini are noted only in the early Eocene. The Oise amber fauna is the most diverse in taxonomic composition and includes four families Anthribidae, Brentidae, Curculionidae and Scolytidae. Only Curculionidae were described from Peckham, London Clay, Havighorst and Mors.

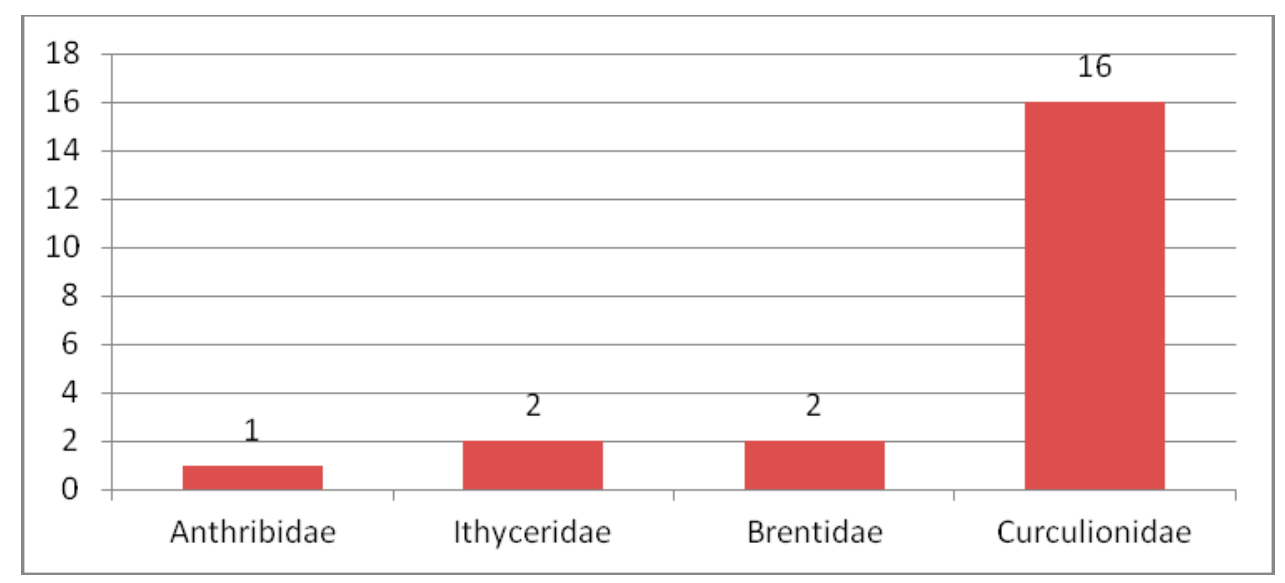

Figure 7. Composition of species of Curculionoidea in the Early Eocene fauna. 


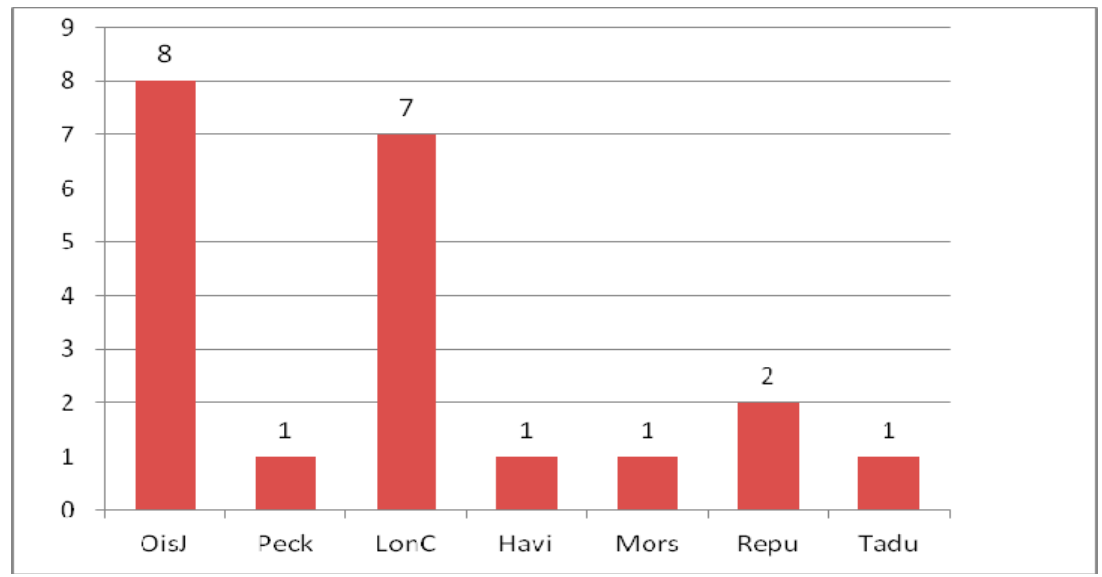

Figure 8. Composition of Curculionoidea species between Early Eocene localities.

The connections between the Paleocene and the early Eocene faunas are in a similar fauna structure with a dominance of Curculionidae and the presence of Ithyceridae. Common genera are not found. Almost all of the early Eocene Curculionidae were associated with trees. The only species which could develop on herbs was "Ceutorhynchus" eocenicus.

\subsubsection{Bridgerian Weevil Fauna}

Within the American localities Green River and Roan Mountain of the Green River Formation (Figure 9), dating from the end of the early to the beginning of middle Eocene, 75 weevil species were described, with six being common to both localities. Two species of the family Nemonychidae, six species of the family Anthribidae, one species of the family Belidae, one species of the family Rhynchitidae, three species of the family Brentidae, 58 species of the family Curculionidae and four species of the family Scolytidae were described (Figure 10). Entiminae dominate the Curculionidae (Figure 11).

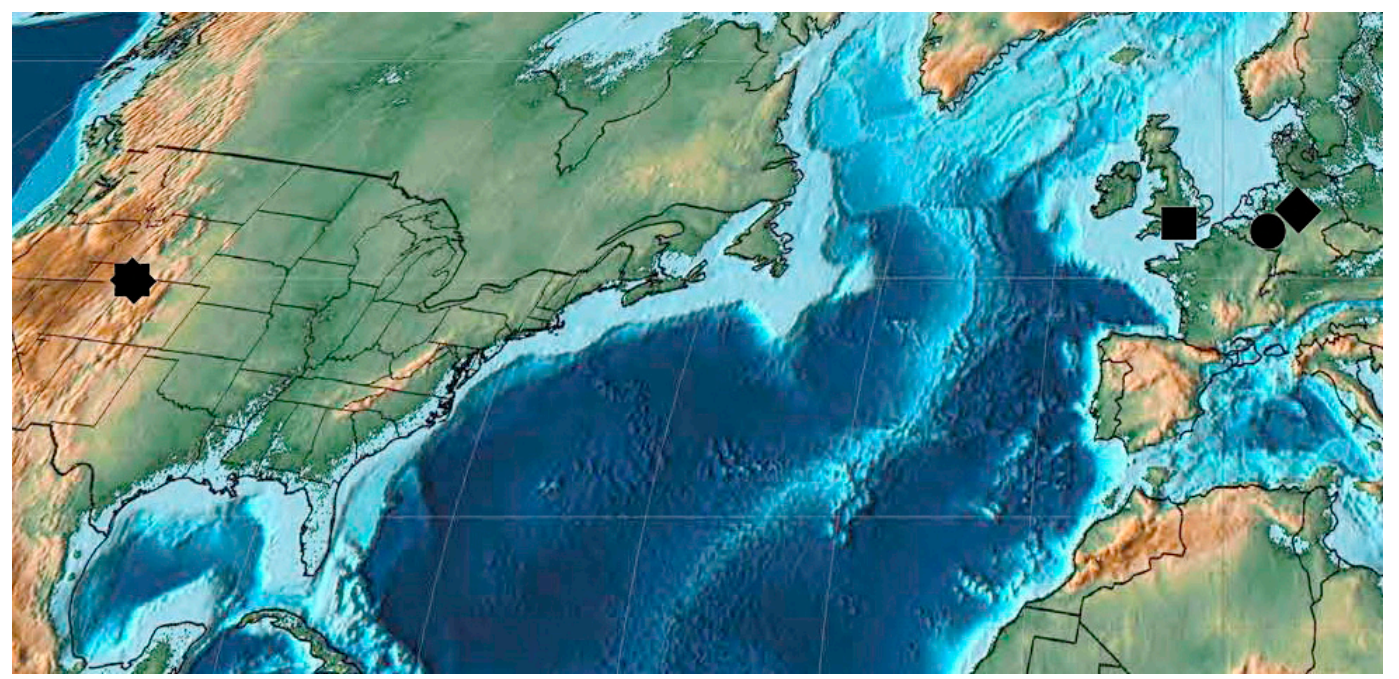

Figure 9. Lutetian Curculionoidea deposits: octagon—Roan Mountain and Green River; square-Corfe and Bournemouth; circle-Messel and Eckfelder Maar, rhombus-Geiseltal. 


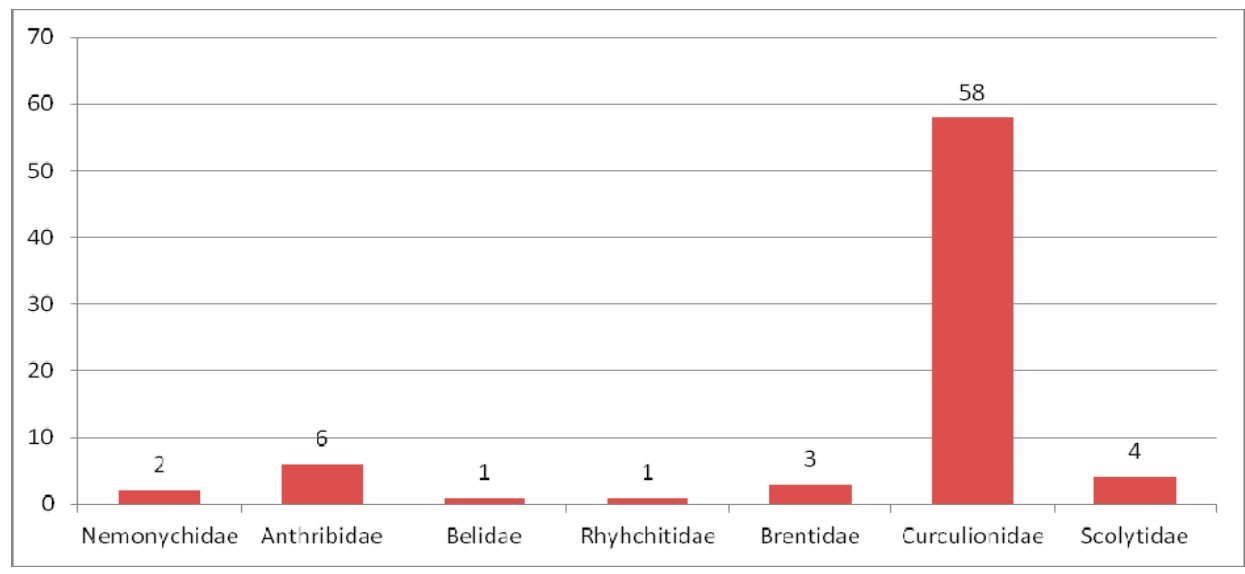

Figure 10. Composition of species of Curculionoidea in the Green River Formation.

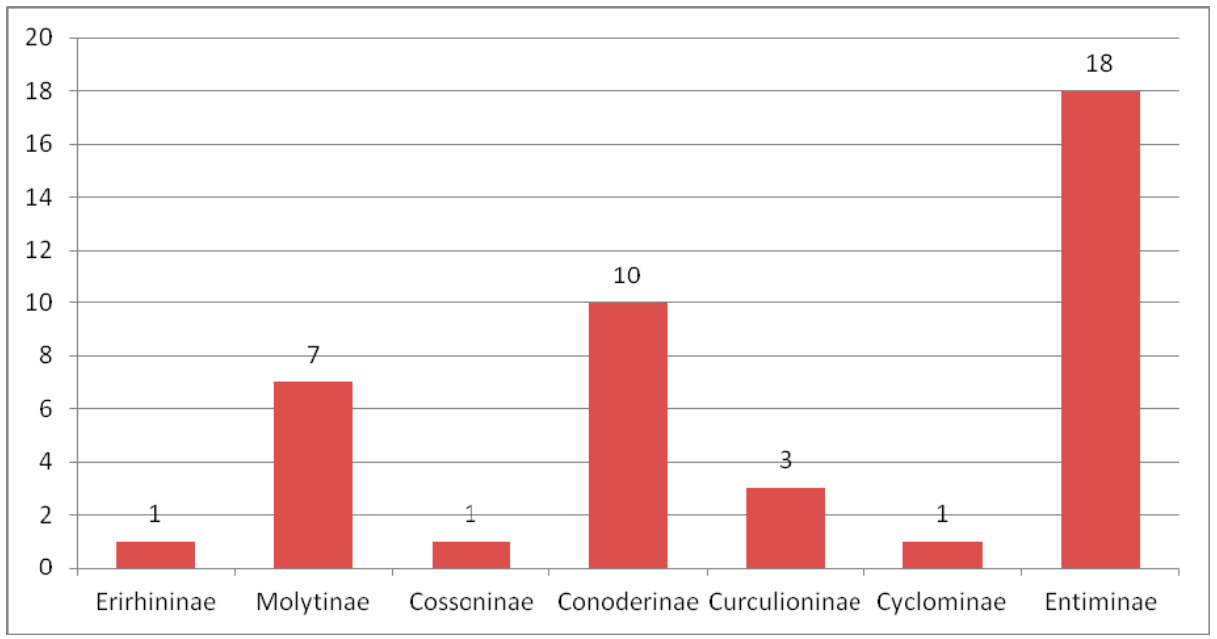

Figure 11. Composition of species of Curculionidae in the Green River Formation.

Here are the earliest records (53.5-48.5 Ma) of the subfamilies Nanophyinae, Conoderinae and Cyclominae, tribes Eocaenonemonychini, Cratoparini, Anthribini, Tropiderini, Choragini, Metrioxenini, Apionini, Apostasimerini, Listroderini, Tropiphorini, Entimini, Sciaphilini, Geonemini, Eustylini and Dryocoetini in the fossil record. The faunas of Green River (51 species) and Roan Mountain (30 species) are very similar. In addition to common species, the generic composition is similar also. Five genera (Sciabregma, Lithogeraeus, Anthonomus, Mitostylus and Epicaerus) are shared by both localities. More samples were collected from the Green River and accordingly more described species. Roan Mountain has a depleted version of the Green River but representatives of the tribes Metrioxenini, Apionini, Erirhinini, Cossonini, Ceutorhynchini and the extinct genus Steganus are known only from this locality. Representatives of Nemonychidae, Anthribidae, Rhynchitidae, Aplemonini, Nanophyinae, Molytini, Cryptorhynchini, Conoderintae, Primocentron, Limalophus, Entimini, Eudiagogini, Eustylini and Dryocoetini are found in the Green River.

The early-middle Eocene faunas of the Green River Formation are quite isolated and do not show obvious connections with other weevil faunas. The genus Perapion connects the Green River fauna with the Paleocene of Menat, the tribe Molytini with the Paleocene of Arkhara, the genera Cossonus and Anthonomus with the Paleocene of Argentina but these widespread groups do not show specificity of the faunogenetic relationships. Two taxa, the genus Sciabregma and the tribe Eudiagogini, show specific relationships between the early Eocene of France and the early-middle Eocene of North America. Most of the Curculionoidea of the Green River were associated with forests. It can be assumed that 
Aplemonini, Nanophyini, Ceutorhynchini, Apostasimerini, Listroderini and possibly some Entiminae lived on riverine meadows.

\subsubsection{Middle Eocene Weevil Fauna}

The middle Eocene consists of two stages, Lutetian and Bartonian. Lutetian localities (Figure 9) are Corfe with one described Curculionoid beetle species, Bournemouth with five species (England), Messel with four species, Geiseltal with six species and Eckfelder Maar with four species (Germany). The systematic position of nine species requires clarification. Here are the earliest records of the subfamily Brentinae $(48.27 \pm 0.22-47.0 \mathrm{Ma})$ and the tribes Baridini (50.0-42.0 Ma), Brachyderini $(48.27 \pm 0.22-47.0 \mathrm{Ma})$ and Sitonini $(47.5-42.5 \mathrm{Ma})$ in the fossil record. Comparison of the fauna of the Lutheian localities among themselves is impossible due to the small number of described forms. Inote that the genera Palaeoalatorostrum, Eckfelderolispa, Cryptorrhynchites, Palaeocrassirhinus, Palaeocneorhinus and Sitonitellus are known only from Lutetian. The richest fauna of Messel and Eckfelder Maar have not yet been described.

Bartonian localities (Figure 12) are Kutschlin (Czech Republic) with one species of the subfamily Molytinae and middle Eocene amber (with 140 species). Specimens in Baltic and Rovno amber are usually separate but an attempt has been Made to compare Baltic amber from individual localities, namely, from Scandinavia, Poland and the Kaliningrad Region. There are very few samples of weevis in Polish and Scandinavian amber. Five species (Baltocyba electrinus, Toxorhynchus michalskii, Baltonanophyes crassirostre, Tachyerges hyperoche and Limalophus poinari) were described from Polish and four species (Archinvolvulus liquidus, Baltoconapium anderseni, Electrotribus henningseni and Ampharthropelma decipiens) from Scandinavian amber were not found in Kaliningrad amber. The tribes Rhadinocybini and Notapionini from the Rhadinocybitae were found only in these ambers, respectively. However, there is no reason to consider these amber separate, since the common species (Electrotribus theryi and Paonaupactus sitonitoides) in Kaliningrad amber were found in Scandinavian and Polish ambers. The absence of species in Kaliningrad amber can be explained by their rarity. Together, 124 species from the families Nemonychidae, Anthribidae, Belidae, Rhynchitidae, Brentidae, Curculionidae, Platypodidae and Scolytidae are recorded fromBaltic amber. The fauna of Rovno amber with 16 species of Curculionoidea has no common species with other amber localities and the two most common species, Electrotribus theryi and Paonaupactus sitonitoides, were not found in Rovno amber. At the generic level, the fauna of Rovno amber is also quite separate since from 13 genera, only 7 genera are common with Baltic amber. All tribes (except Valenfriesiini) recorded in Rovno amber were also found in Baltic amber. Representatives of eight families were found in Rovno and Baltic amber (Figure 13). A detailed analysis of the faunas of Eocene ambers was carried out in other articles [71,191]. In Eocene amber are the first records of the subfamily Tesserocerinae, the supertribes Rhadinocybitae and Aspidapiitae, the tribes Mecocerini, Allandrini, Zygaenodini, Oxycraspedini, Piezotrachelini, Dorytomini, Plinthini, Acicnemidini, Stromboscerini, Dryotribini, Ellescini, Eugnomini, Rhamphini, Camarotini, Hyperini, Trachyphloeini, Anypotactini, Polydrusini, Hylastini, Hylurgini, Phloeosinini and Polygraphini and the last find of the Sayrevilleinae subfamily in the fossil record. The faunas of Bartonian are very different from the fauna of Lutetian. Common genera are absent. 


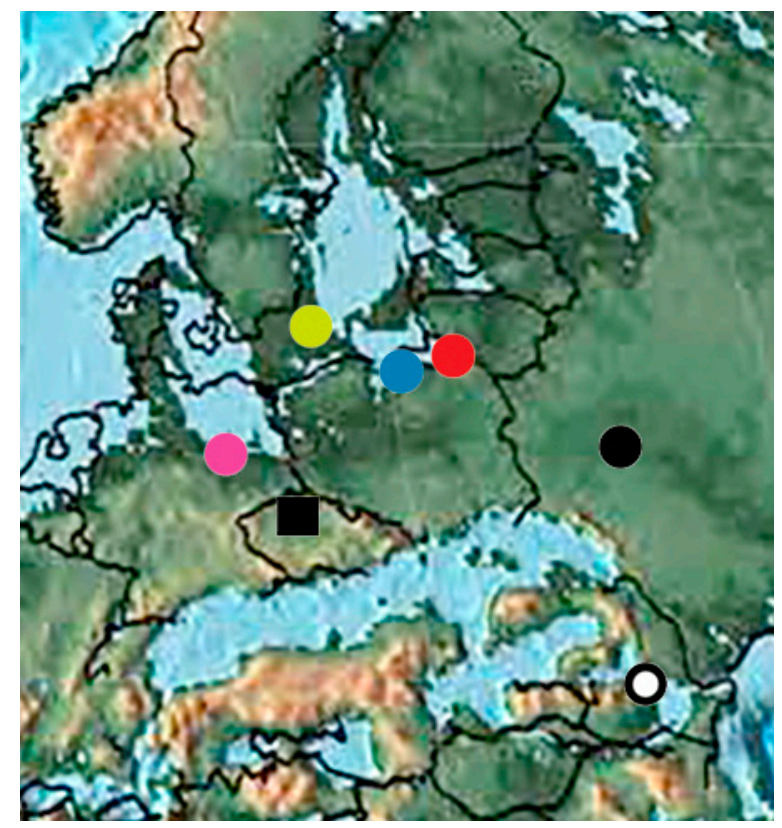

Figure 12. Bartonian Curculionoidea deposits: red circle-Baltic amber (Kaliningrad region); blue circle-Polish amber; yellow circle-Scandinavian amber; pink circle-Bitterfeld amber; ring-Romanian amber; circle-Rovno amber; square-Kutschlin.

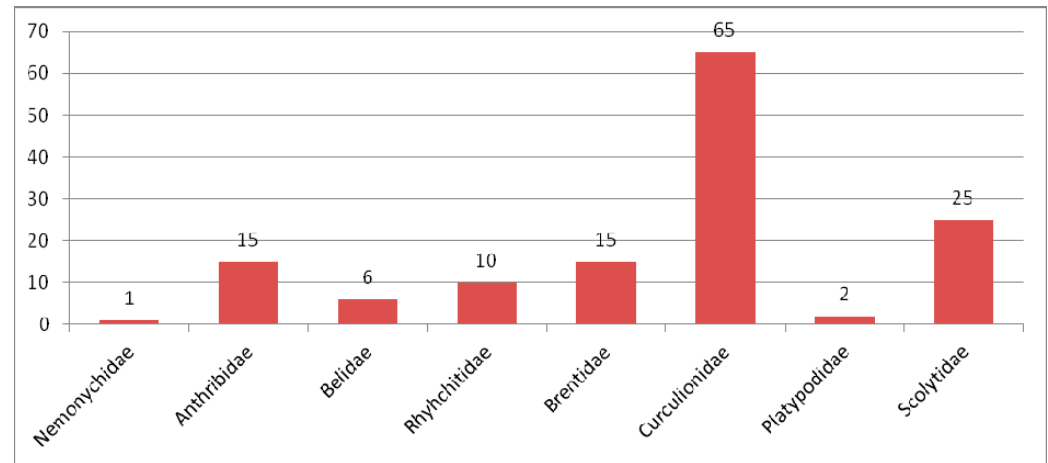

Figure 13. Composition of species of Curculionoidea in the Baltic amber.

In general, the weevil fauna of the middle Eocene of Europe is very distinct from earlier faunas. The connections between it and the North American Ypresian-Luthean faunas includethe subtribe Zherichinixenina of the tribe Metrioxenini and the genus Limalophus, in both of them. The similarity with the Ypresian and Paleocene faunas of Europe lies only in the presence of widespread groups at the level of tribes (Cryptorhynchini, Ceutorhynchini, Curculionini and Naupactini) and subfamilies (Anthribinae, Apioninae, Molytinae, Dryophthorinae, Cossoninae, Curculioninae).

\subsubsection{Late Eocene Weevil Fauna}

The Priabonian faunas are described from five localities (Figure 14), two in France (Celas and Ales-Monteils), one in the USA (Florissant), one in the south of the Russian Far East (Biamo) and one in Brazil (Fonseca). Some 184 species were described from these localities. One species of the tribe Hyperini was described from Biamo. The genus Duartia, probably related to Scolytidae, is known from Fonseca. Palaeorhopalotria neli was described from Ales-Monteils. This species belongs to the extinct tribe Palaeorhopalotriini belonging to the supertribe Allocorynitae now distributed in Central America [192] and also noted in the Miocene [193]. The three representatives of Entiminae and species from the tribe Corimaliini (earliest record) are known from Celas. 


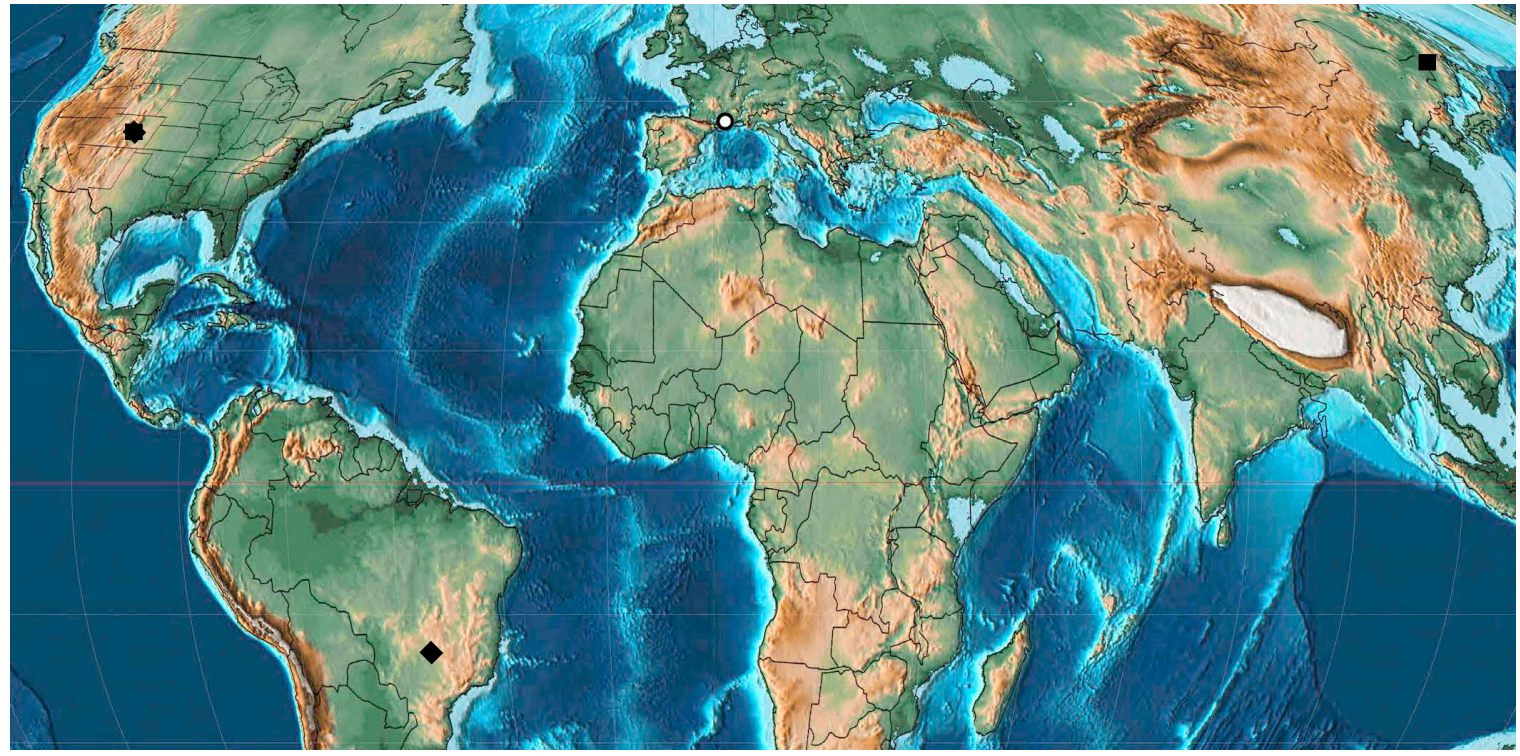

Figure 14. Priabonian Curculionoidea deposits: Circle-Celas and Ales-Monteils; octagon-Florissant; square-Biamo; rhombus-Fonseca.

The Florissant fauna, from which 177 species are known (Figure 15), is of great interest as being the richest of the Paleogene fauna. However, families Nemonychidae, Ithyceridae and Platypodidae are absent in this fauna. Representatives of the tribes Cratoparini, Anthribini, Ecelonerini, Tropiderini, Metrioxenini, Auletini, Rhynchitini, Eugnamptini (earliest record), Euscelini (earliest record), Apionini, Erirhinini, Dorytomini, Molytini, Magdalini (earliest record), Cryptorhynchini, Cleonini (earliest record), Dryophthorini, Sphenophorini (earliest record), Cossonini, Apostasimerini, Baridini, Ceutorhynchini, Cnemogonini (earliest record), Curculionini, Anthonomini, Rhamphini, Tychiini, Camarotini, Listroderini, Tropiphorini, Eudiagogini, Hyperini, Hormorini (earliest record), Naupactini, Geonemini, Psallidiini (earliest record), Tanymecini (earliest record), Hylastini and Phloeotribini (earliest record) are found in Florissant deposits (34.07 $\pm 0.10 \mathrm{Ma})$.

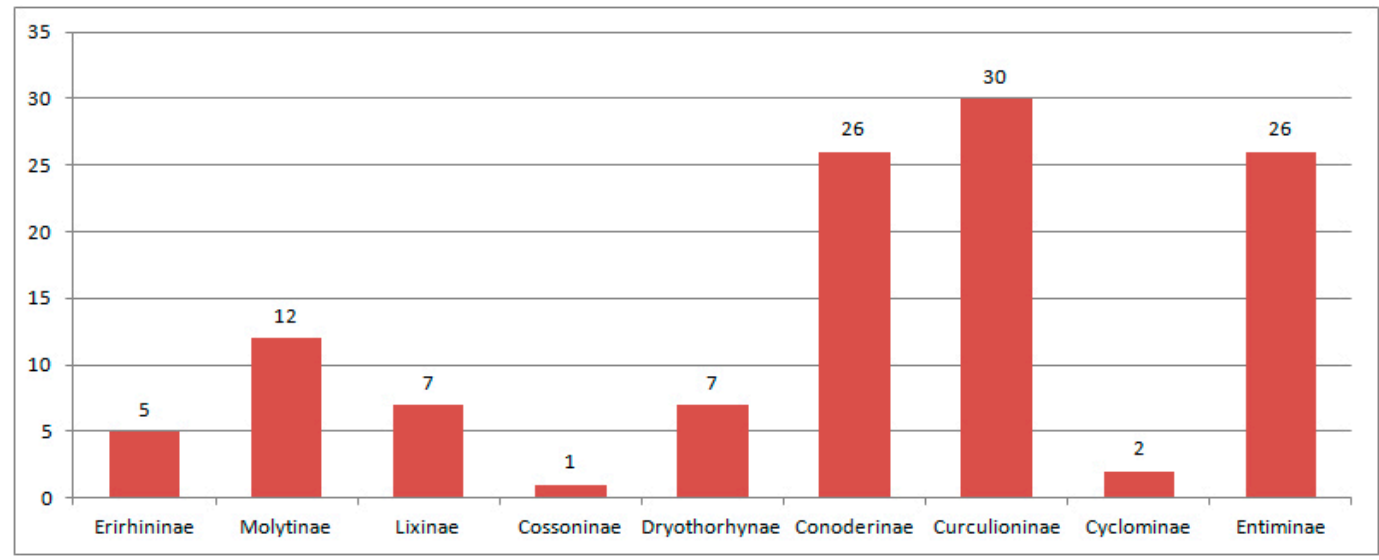

Figure 15. Composition of species of Curculionoidea in the Florissant.

About half (52\%) of the genera are recent. This fauna of Florissant is very different from the fauna of Barton amber, where $70 \%$ of genera are extinct. The structure of the fauna is rather unusual (Figure 15). Curculionidae dominates but Rhynchitidae and Brentidae also play an important role. Three subfamilies (Curculioninae, Conoderinae and Entiminae) of the weevils form the basis of the fauna (Figure 16), while Curculioninae and Entiminae and Conoderinae do not play a significant role (Figure 17) in Baltic amber. It differs from the fauna of the Green River, where only Curculionidae 
dominates and at the subfamily level of this family, the fauna is formed by Entiminae and Conoderinae. However, due to the presence of common groups (genera and tribes, usually found only in these localities), the Florissant fauna shows similarities primarily with the Green River (15 common genera and tribes) and Baltic and Rovno amber (11 common genera and tribes) faunas.

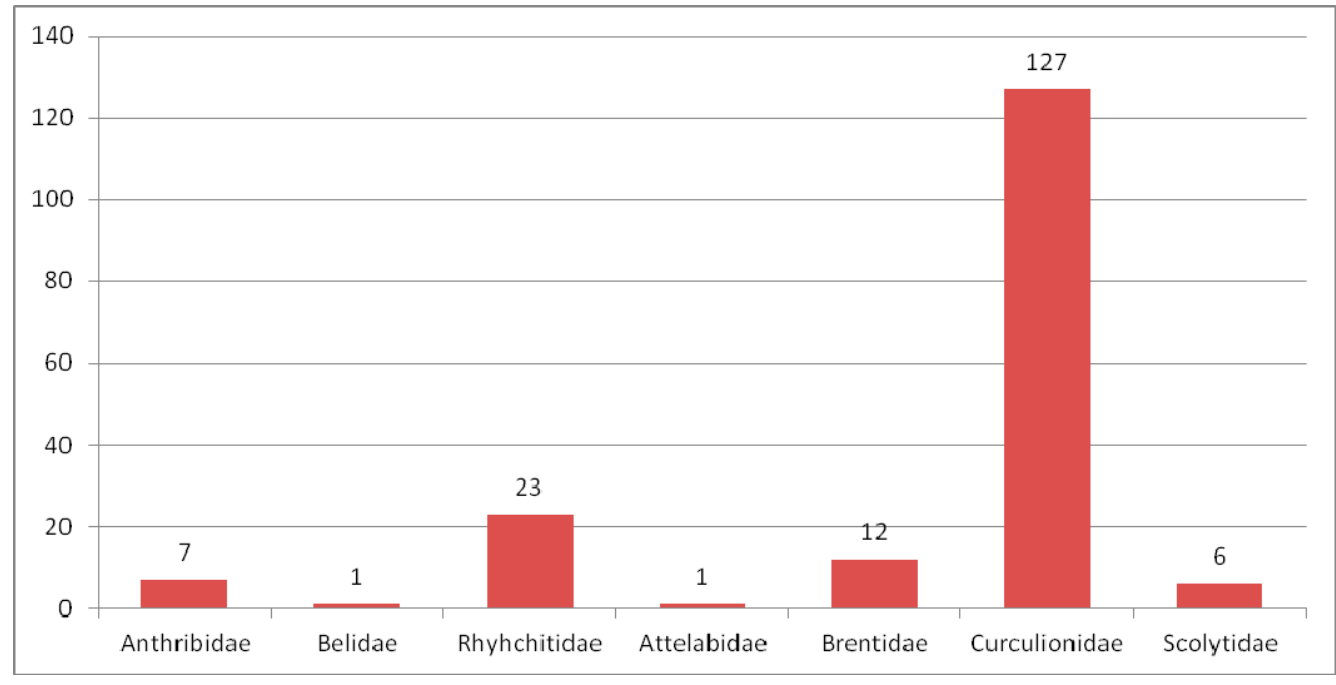

Figure 16. Composition of species of Curculionidae in the Florissant.

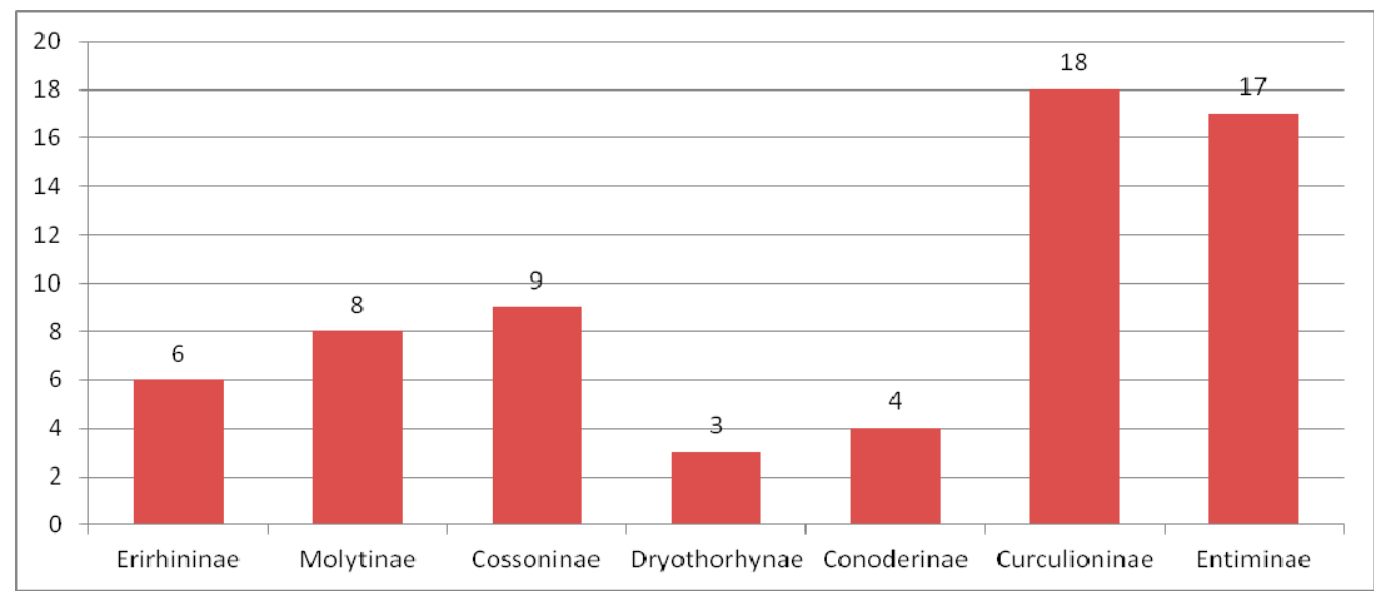

Figure 17. Composition of species of Curculionidae in the Baltic amber.

\subsubsection{Comparison of the Eocene Weevil Faunas}

In the Eocene, weevil diversity occurs from early to late (Figures 18 and 19). A small number of species in the Lutetian is due to poorly studied fauna. The number of representatives of modern genera increases towards the late Eocene. 


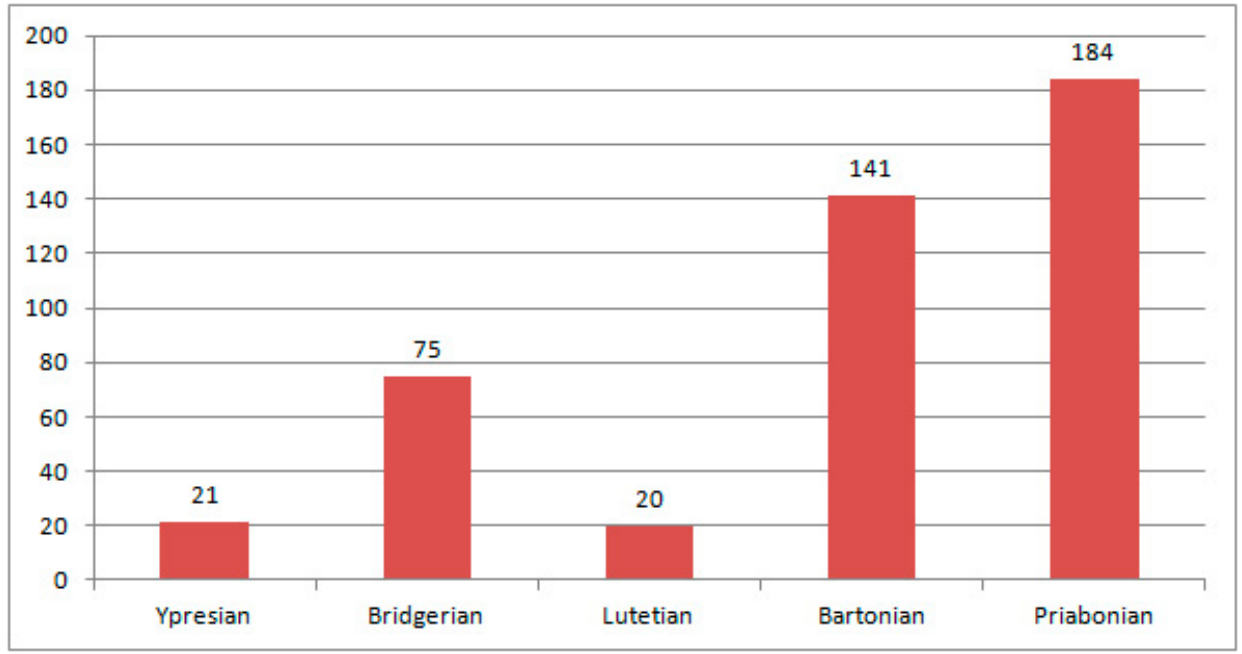

Figure 18. Change in the number of Curculionoidea species in the Eocene.

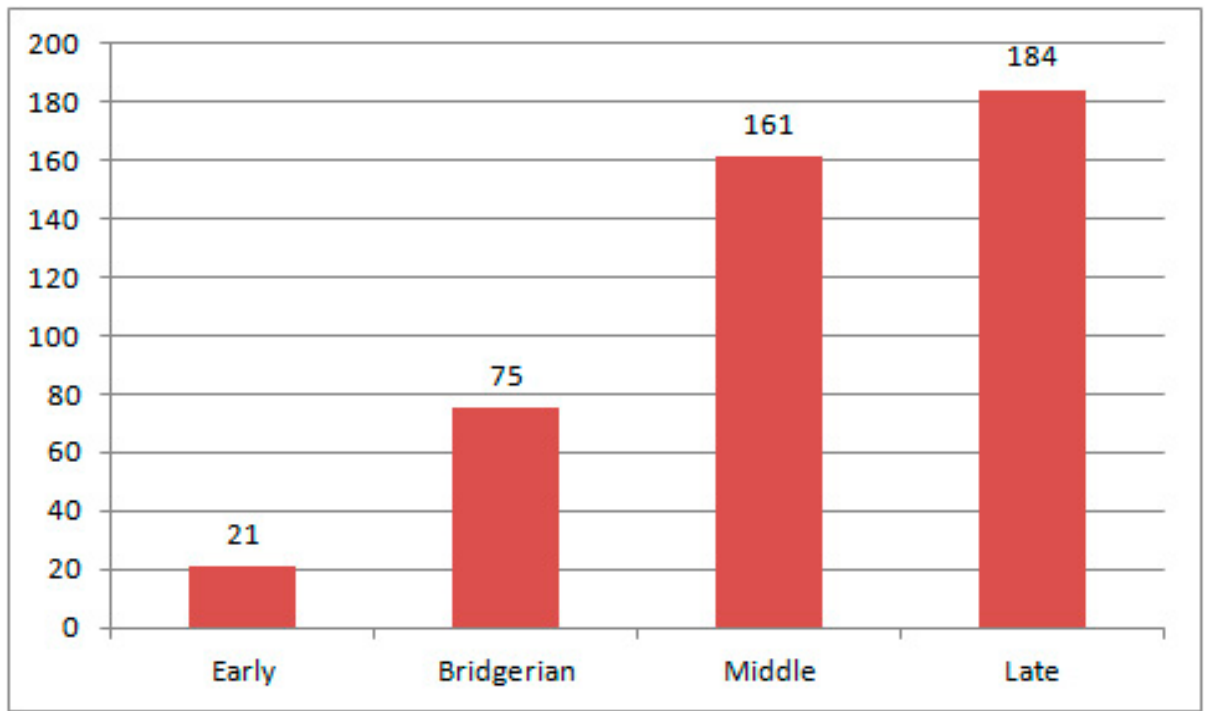

Figure 19. Change in the number of Curculionoidea species in the Eocene.

\subsection{Oligocene Weevil Fauna}

The Oligocene fauna of Curculionoid beetles is represented by 94 described species from six families. Ten localities related to the early (White River Badlands from USA, Brunnstatt and Corent from France), middle (border between early and late) (Sieblos and Kleinkembs from Germany, Gaube and Puy-Saint-Jean from France) and late Oligocene (Luzice from Czech Republic, Aix-en-Provance from France, Rott from Germany) (Figures 20 and 21), contain the remains of Curculionoidea that were described. The Greenland locality of Kap Dalton, where representative of the Nemonychidae family was described, dates from the Oligocene without specifying the stage [194]. Curculionoidea are also represented in the Rupelian Quilchena (Canada), Cereste (France), Seifhennersdorf (Germany), Ahuehuetes (Mexico), Kundratice (Czech Republic), Hutt Enspel (Germany), Ashutas (Kazakhstan), Perekishkyul' (Azerbaijan) localities [30,195-199]. 


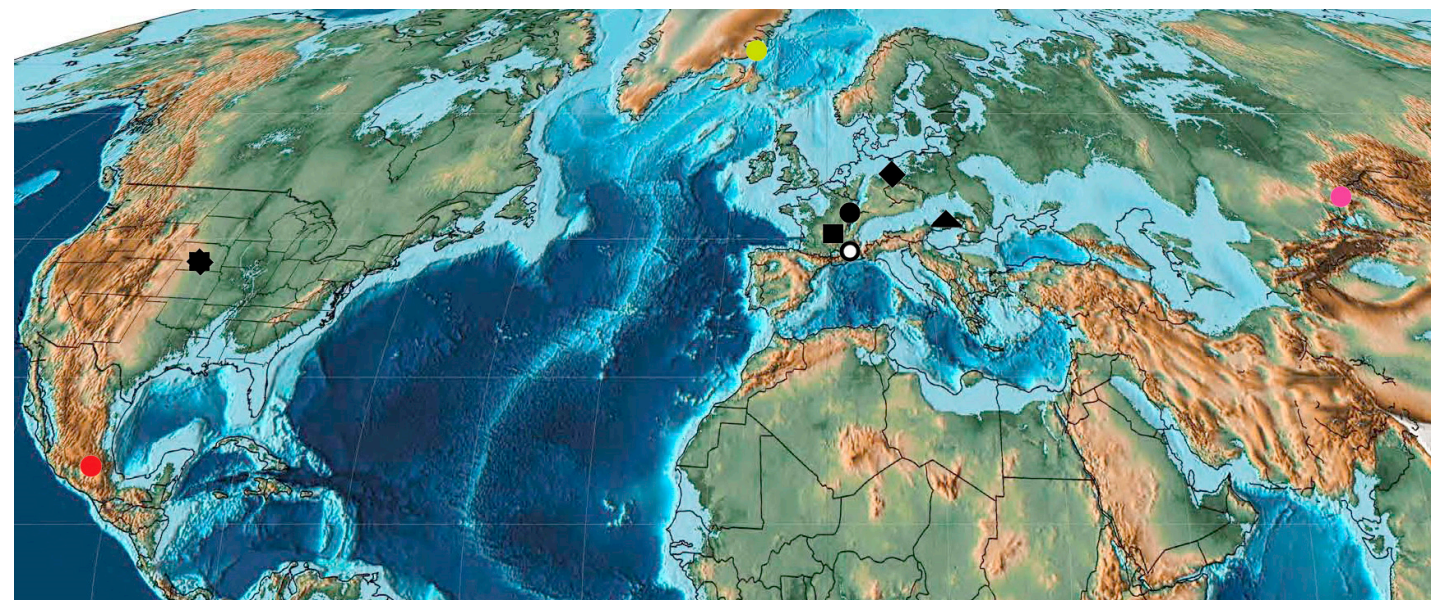

Figure 20. Rupelian Curculionoidea deposits: octagon-White River Badlands; circle-Brunnstatt; square-Corent; ring-Cereste; rhombus-Seifhennersdorf; red circle-Ahuehuetes; triangle-Kundratice; yellow circle-Kap Dalton; pink circle-Kenderlyk II.

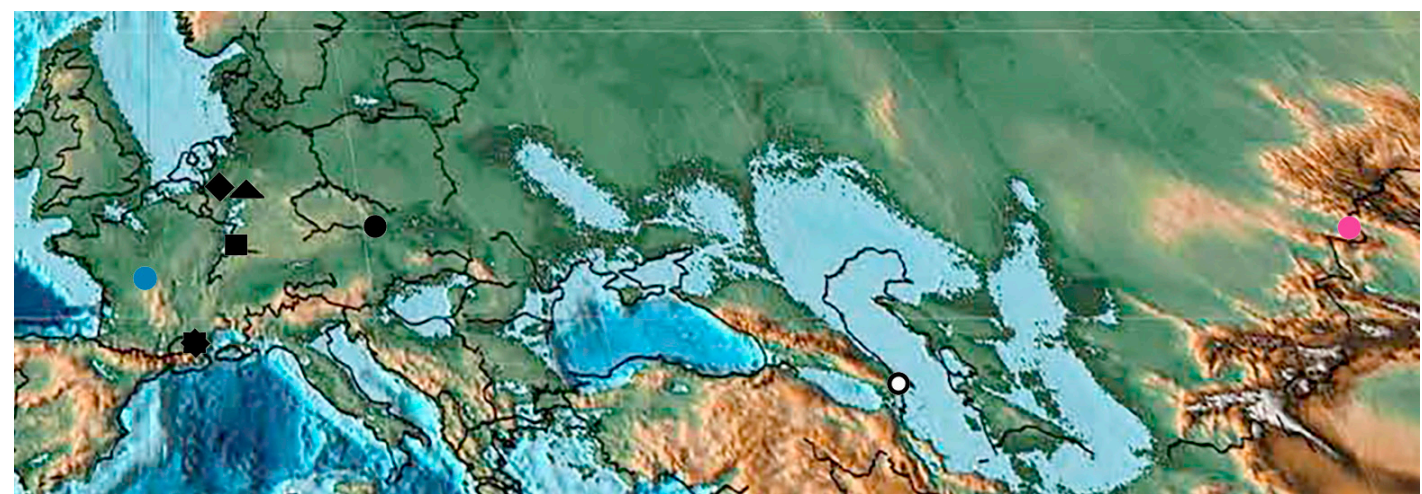

Figure 21. Middle and late Oligocene Curculionoidea deposits: circle—Luzice; rhombus—Rott and Enspel; octagon-Aix-en-Provance; pink circle-Ashutas; ring-Perekishkyul'; triangle-Sieblos; square-Kleinkembs; blue circle—Gaube and Puy-Saint-Jean.

Representatives of the family Curculionidae and a few species of the subfamily Apioninae from the family Brentidae were found in these localities.

\subsubsection{Review of Curculionoidea Families in the Oligocene}

Nemonychidae in the Oligocene

The family Nemonychidae in the Oligocene is represented by one species assigned to the tribe Eocaenonemonychini from the subfamily Cretonemonychinae. This is the latest find of the family in the fossil record.

\section{Anthribidae in the Oligocene}

The family Anthribidae is very poorly represented in Oligocene deposits (Figure 22). No representatives of the subfamily Anthribinae were found. One species of the tribe Choragini was described from the late Oligocene. Choraginae were very rare in the Eocene, so this find shows a greater abundance of this subfamily in the Oligocene. Three species of the genus Bruchela of the subfamily Urodontinae were described from the Oligocene of France and Germany. This is the first reliable indication of Urodontinae in the fossil record. 


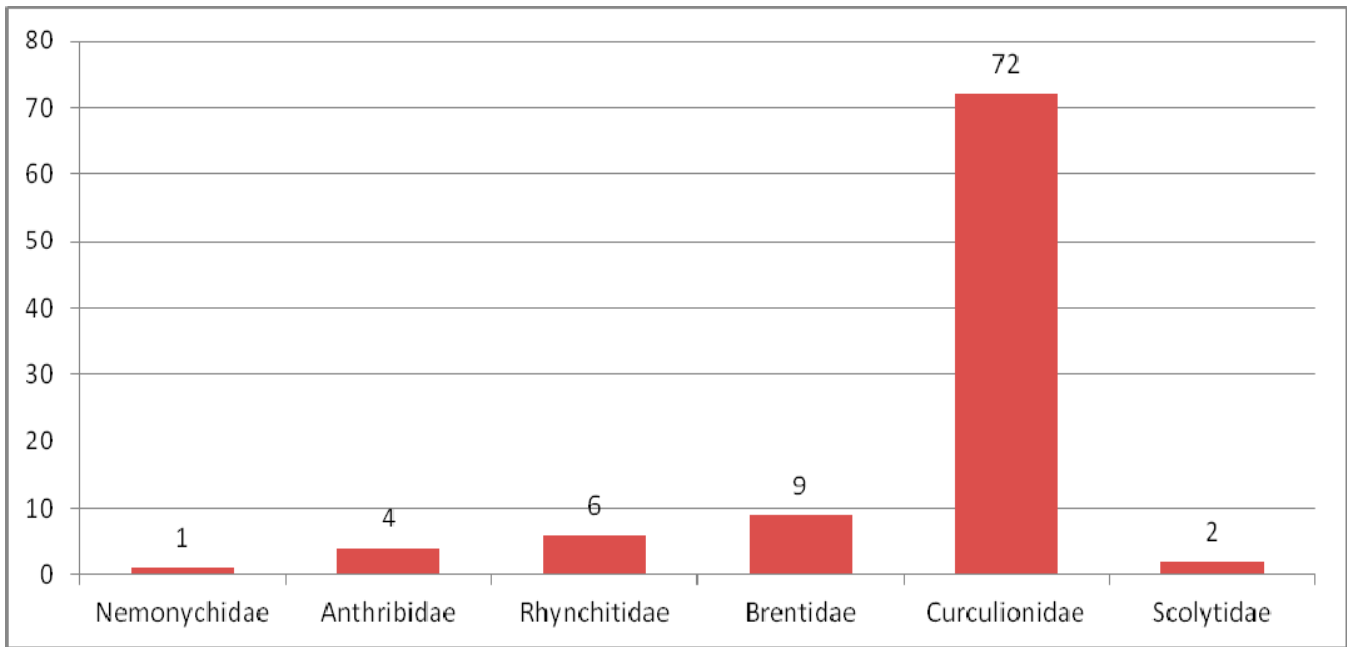

Figure 22. Composition of species of Curculionoidea in the Oligocene.

Rhynchitidae in the Oligocene

Six species of the family Rhynchitidae occur in the late Oligocene. The extinct genus of the tribe Vossicartini, now distributed only in tropical Africa and Madagascar [60], is the only find of this tribe in the fossil record. The tribe Rhynchitini is represented by extinct species of recent genera belonging to both the primitive subtribe Perrhynchitina and the advanced subtribe Rhynchitina.

Attelabidae in the Oligocene

The Attelabidae family was not found in the Oligocene.

\section{Brentidae in the Oligocene}

The family Brentidae is found in the Oligocene of Europe. Eight species belong to the subfamily Apioninae and one to the subfamily Nanophyinae (tribe Nanophyini). The systematic position of most species in the tribes and genera requires clarification. One species can be assigned to the genus Perapion of the tribe Aplemonini. Previously, this genus was discovered in the Paleocene of Europe and the Eocene of North America.

Curculionidae in the Oligocene

The Curculionidae family is represented by 72 species from the subfamilies Erirhininae, Molytinae, Lixinae, Dryophthorinae, Cossoninae, Conoderinae, Curculioninae and Entiminae (Figure 23). Only the subfamily Cyclominae was not encounteredin Oligocene deposits. Two species from the tribes Erirhinini and Bagoini of the subfamily Erirhininae are noted in the Oligocene. Representatives of the tribes Molytini, Pissodini, Magdalini and Cryptorhynchini belonging to the subfamily Molytinae were described from different Oligocene localities. Lithopissodes luschitzensis is the only Pissodini in the fossil record. Two species of the genus Larinus of the tribe Lixini and two species formally assigned to the genus Cleonis belong to the subfamily Lixinae. The subfamily Dryophthorinae with one species of the tribe Dryophthorini and one species of the tribe Sphenophorini is known from the late Oligocene. Here is the only record of the tribe Sphenophorini in the Paleogene of Europe. Only two species from the tribes Rhyncolini and Cossonini of the subfamily Cossoninae were found in the late Oligocene. It is important to note that the tribe Dryotribini common in the middle Eocene amber are not found in the Oligocene. The diverse subfamily Conoderinae is poorly represented in the Oligocene. Two species of Bariditae are noted. Lithogeraeus comminute from the tribe Apostasimerini was described from the early Oligocene of the United States. One species placed in the genus Baris of the tribe Baridini is known from the early Oligocene of Europe. The supertribe Conoderintae is not found in the 
Oligocene. The supertribe Ceutorhynchitae is represented by the tribes Ceutorhynchini and Phytobiini. The tribes Smicronychini, Anthonomini and Tychiini from the subfamily Curculioninae are found in the Oligocene. "Smicronyx" antiquus is the only species of the tribe Smicronychini in the fossil record. "Anthonomus" soporus from the tribe Anthonomini is Marked for the early Oligocene of the United States. The Oligocene species of the genera Sibinia and Tychius belong to the tribe Tychiini. Twenty three species from six tribes belong to the subfamily Entiminae. The most diverse tribes are Tropiphorini with 7 species and Tanymecini with 6 species. One or two species belong to the tribes Sciaphilini, Sitonini, Geonemini and Brachyderini. The position of four species in these tribes requires clarification. The family Platypodidae is not found in the Oligocene. Two species of the genus Hylesinus of the tribe Hylesinini from the family Scolytidae are known from the late Oligocene of France.

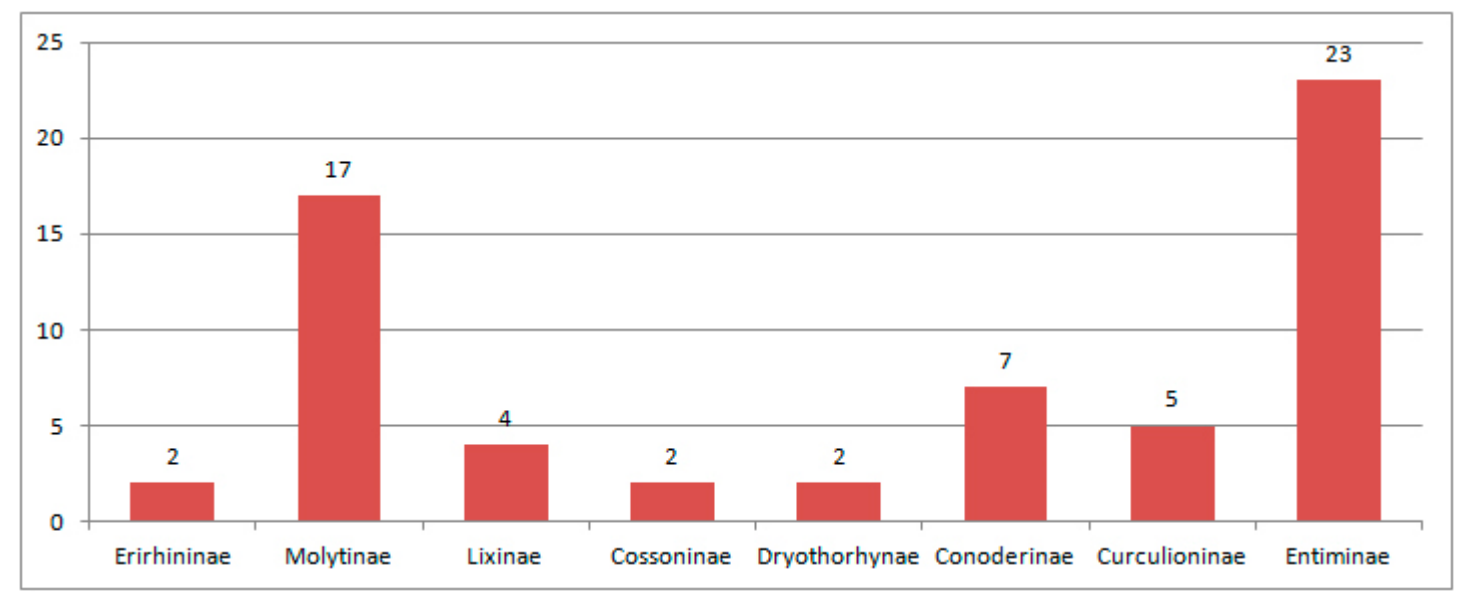

Figure 23. Composition of species of Curculionidae in the Oligocene.

Localities with the described fauna are divided into those located in North America and Europe. Curculionidae from the only Asian locality in Kazakhstan have not yet been described. Species were described from the White River Badlands and Kap Dalton in Greenland. Their fauna is radically different from the fauna of the Old World. At least 13 species of the family Curculionidae are known from the White River Badlands. These are representatives of the genera Procas, Archaeoheilus, Lithogeraeus, Anthonomus, Limalophus, Mitostylus and Epicaerus. None of these genera were found in the Oligocene of Europe. The fauna of this locality shows a close similarity with the fauna of the middle Eocene, Green River and Road Mountain. Moreover, three species from the White River are found inthese faunas. At the level of genera and tribes, the similarity between them is complete. One can consider White River as a reduced version of the Eocene American faunas. The only Curculionoidea from the Oligocene of Greenland belongs to the North American tribe Eocaenonemonychini and possibly to a genus described from the Green River.

\subsubsection{Early Oligocene Weevil Fauna}

Twenty one species are found in the early Oligocene of Europe (France), 13 species in Brunnstatt and 8 species in Corent. There is one species of the subfamily Urodintinae (Anthribidae), three species of the subfamily Apioninae (Brentidae), 17 species of Curculionidae (one species of the subfamily Erirhininae, five species of the subfamily Molytinae, three species of the subfamily Lixinae, four species of the subfamily Conoderinae, two species of the subfamily Curculioninae, one species of the subfamily Entiminae and one species of the subfamily insertae sedis). The faunas of Brunnstatt and Corent are very different. No species and genera occur in both localities. Three species in Corent can be attributed to the meadow complex (Anisorhynchus and Cleonini) and three to the forest complex (representatives of Molytinae). The fauna of Brunnstatt is much more diverse. Most species noted there belong to the meadow-steppe complex (11 species from the genera Bruchela, Larinus, Baris, Ceutrhynchus, Smicronyx, 
Tychius and the subfamily Apioninae) while Molytinae probably belonged to the forest complex and Bagous to the near-water habitat.

\subsubsection{Middle Oligocene Weevil Fauna}

Only ten weevil species were described from the middle Oligocene (boundaries are of the early and late Oligocene) and six of them belong to Curculionidae insertae sedis. The rest belong to the subfamilies Molytinae, Conoderinae and Curculioninae of the family Curculionidae.

\subsubsection{Late Oligocene Weevil Fauna}

Thirty four species were described from the late Oligocene. One species is known from Luzice, 22 from Aix-en-Provance and 27 from Rott. The fauna of Aix-en-Provance is represented by the three families, Brentidae, Curculionidae and Scolytidae (Figure 24). They involveforest species (Dryophthorus incertus, Cossonus robustus, Rhytidoderes spp., Hylesinus spp.) and open space species (Apioninae, "Sibinia" whitneyi), Protainophthalmus spp. Extinct species belong to widespread groups. Noteworthy are six species of the extinct genus Protainophthalmus known only from this locality. This genus belongs to the subtribe Tainophthalmina that is distributed in Central and East Asia and is also found in the Mediterranean. Three extinct species of the recent genus Rhytideres from the Mediterranean are also only found in Aix-en-Provance. The families Anthribidae, Rhynchitidae, Brentidae and Curculionidae form the Rott fauna (Figure 25). Species of the family Curculionidae dominate. Most species belong to widespread genera (Choragus, Perapion, Nanophyes, Hylobius, Magdalis, Acalles, Larinus, Sphenophorus, Rhyncolus, Ceutorhynchus and Tychius). Three species belong to the West Palaearctic genera Bruchela and Tatianaerhynchites. Representatives of the oriental genera Cartorhynchites and Opacoinvolvulus are also present. The two genera Germanocartus and Brachymycterus are endemic to this locality. The Rott fauna consisted of approximately half forest and meadow species. It is obviousthat the Rott fauna is much more diverse than that of Aix-en-Provance. Common genera are absent.

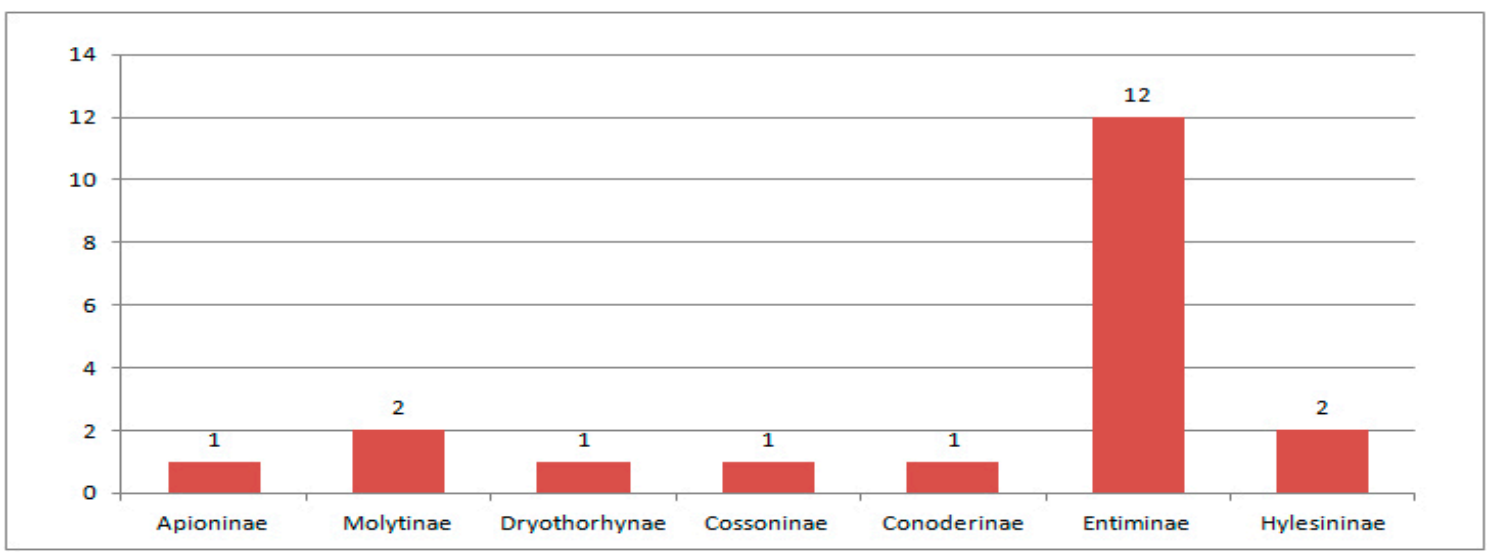

Figure 24. Composition of species of Curculionoidea in Aix-en-Provance. 


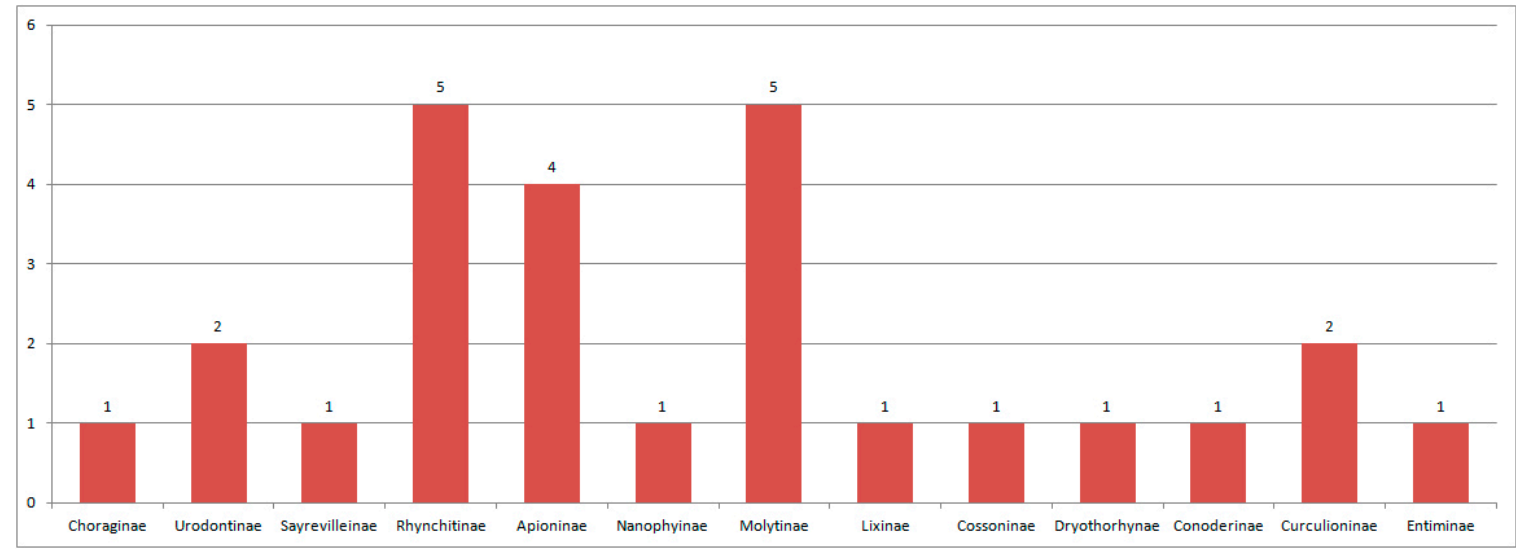

Figure 25. Composition of species of Curculionoidea in Rott.

\subsubsection{Comparing of the Oligocene Weevil Faunas}

Comparing the faunas of the early and late Oligocene, it is noted that the Chattian faunas are more diverse. They contain not only representatives of widespread or West Palaearctic genera but also oriental forms. Relationships with the African fauna are indicated the presence of a representative of the tribe Vossicartini. In general, the Oligocene fauna was formed by forest and meadow-steppe species, which indicates the presence of open spaces.

\section{Discussion}

In total, 564 species of Curculionoidea from nine families were described from the Paleogene. They occur in seven localities in North America (four from Eocene and three from Oligocene), three in South America (one from the Paleocene and two from the Eocene), 34 in Europe (two from the Paleocene, 18 from the Eocene and 14 from the Oligocene) and six in North and East Asia (two from each of the Paleocene, the Eocene and the Oligocene). They have not yet been found in African, South Asian and Australian Paleogene localities. An increase in the number of species is observed from the Paleocene to the Eocene (end of the Eocene) and then decreases in the Oligocene (Figure 26). The greatest diversity of Curculionoidea is described from the Eocene of Europe and North America. The richest faunas are known from the terminal Eocene of Florissant (177 species), the middle Eocene Baltic amber (124 species) and the early-middle Eocene Green River formation (75 species). Discovery of relict groups with a local distribution in the contemporary fauna, such as Ithyceridae-Chilecarinae and Ithycerinae, Belidae-Metrioxenini, Rhynchitinae-Sayrevilleinae, were Made in the Eocene of America and the Eocene and Oligocene of Europe. The most numerous group of all the Paleogene Coleoptera faunas is the superfamily Curculionoidea [200]. The family Curculionidae dominates in all localities of the Paleogene. Anthribidae, Rhynchitidae, Brentidae and Scolytidae are sometimes subdominant in Eocene localities. For example, Anthribidae, Brentidae and Scolytidae account for $40 \%$ of the Baltic amber fauna (weevils $47 \%$ ) and Rhynchitidae comprise $13 \%$ of the Florissant fauna (weevils $72 \%$ ). In all localities, species associated with woody vegetation dominate. Species associated with herbaceous vegetation are present in most localities since the middle Paleocene. Their proportion is increasing in the Oligocene. Further study of the Curculionoidea from Paleogene localities May clarify the picture somewhat. 


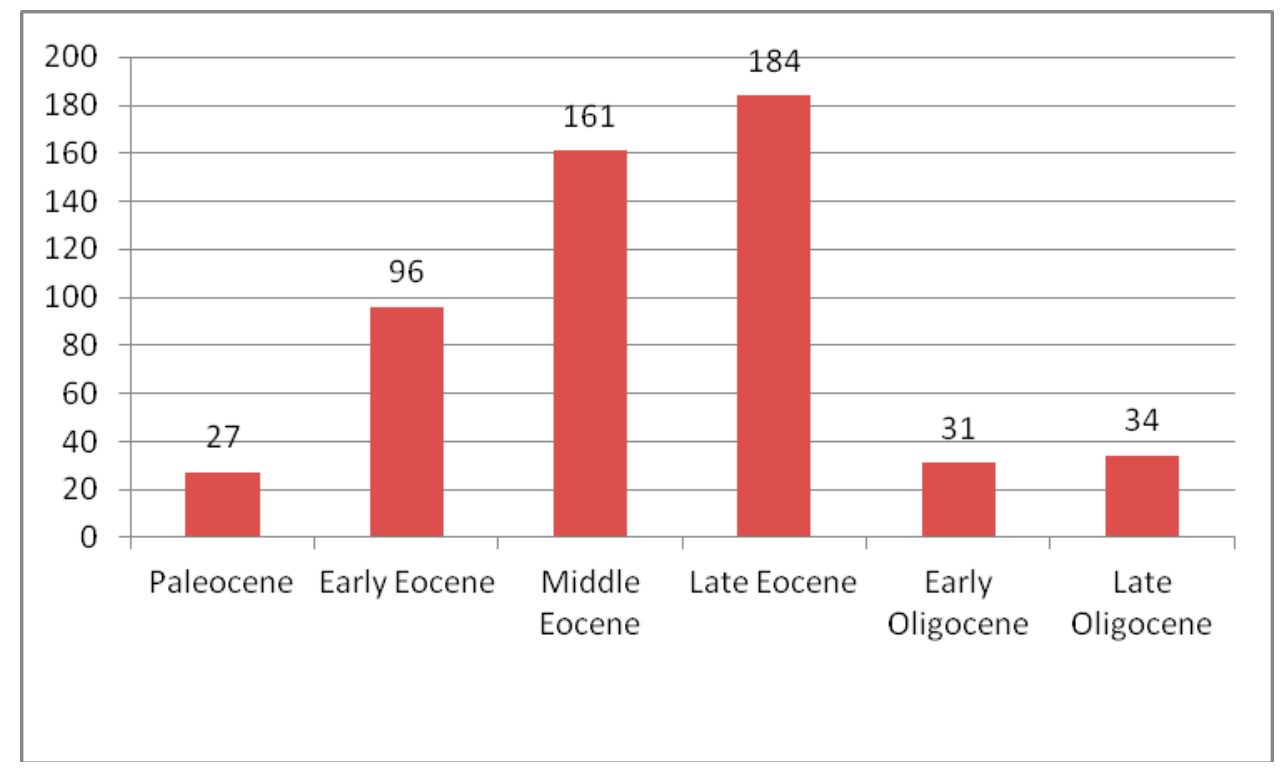

Figure 26. Change in number of Curculionoidea species in the Paleogene subepochs. The fauna of the Green River Formation is included in the Early Eocene.

Funding: The study was partially supported by the Russian Foundation for Basic Research (project nos. 18-04-00243-a and 19-04-00465-a) and the Federal Fundamental Scientific Research Program for 2013-2020 (project no. AAAA-A16-116121410121-7).

Acknowledgments: The author thanks Many colleagues for their assistance throughout his studies: V.I. Alekseev, A.R. Manukyan and A.V. Smirnova (Kaliningrad Regional Amber Museum, Russia: Kaliningrad), A. Allen (USA: Boise), A. Bukejs (Institute of Life Sciences and Technologies, Daugavpils University, Latvia: Daugavpils), D. Berthet (Centre de Conservation du musée des confluences, France, Lyon), J. Damzen (Lithuania: Vilnius), F. Eichmann (Germany: Hannover), A. Górski (Poland: Bielsko-Biaùa), C. Gröhn (Germany: Glinde), V.A. Gusakov (Russia: Moscow), A.G. Kirejtshuk (Zoological Institute RAS, Russia: St. Petersburg), U. Kotthoff (Center of Natural History, Germany: Hamburg), N.V. Martynovich (Museum of the World Ocean, Russia: Kaliningrad), A. Nel (Muséum national d'histoire naturelle, France: Paris), E.E. Perkovsky and V.Y. Nazarenko (Schmalhausen Institute of Zoology, NASU, Ukraine: Kiev), A.G. Ponomarenko, A.P. Rasnitsyn, E.D. Lukashevich, D.E. Shcherbakov, I.D. Sukatsheva, D.V. Vassilenko (Borissiak Paleontological Institute RAS, Russia: Moscow), E. Yu. Shevnin (Russia: Novosibirsk), L.B. Vilhelmsen (Zoological Museum, University of Copenhagen, Denmark: Copenhagen), K. Szczepaniak (Earth Institute, Poland: Warsaw), T. Wappler (Hessisches Landesmuseum Darmstadt, Germany: Darmstadt), Ch. R. Scotese (Northwestern University, USA: Evanston) allowing me to use his paleomaps and G.O. Poinar, Jr (Oregon State University, USA: Corvallis) for improving the Manuscript. The two reviewers are also acknowledged for the valuable comments that improved the manuscript.

Conflicts of Interest: The author declares no conflict of interest.

\section{References}

1. Scotese, C.R. Atlas of Paleogene Paleogeographic Maps (Mollweide Projection), Maps 8-15, Volume 1, The Cenozoic, PALEOMAP Atlas for ArcGIS, PALEOMAP Project, Evanston, IL. 2014. Available online: https://www.researchgate.net/publication/267569784_Atlas_of_Paleogene_ Paleogeographic_Maps_Mollweide_Projection_Maps_8-15_Volume_1_The_Cenozoic_PALEOMAP_ Atlas_for_ArcGIS_PALEOMAP_Project_Evanston_IL (accessed on 6 September 2020).

2. Blythe, A.E.; Kleinspehn, K.L. Tectonically versus climatically driven Cenozoic exhumation of the Eurasian plate Margin, Svalbard: Fission track analyses. Tectonics 1998, 17, 621-639. [CrossRef]

3. Nel, A. The oldest bee fly in the French Paleocene (Diptera: Bombyliidae). C.R.Palevol 2008, 7, 401-405. [CrossRef]

4. Wappler, T.; Currano, E.D.; Wilf, P.; Rust, J.; Labandeira, C.C. No post-Cretaceous ecosystem depression in European forests? Rich insect feeding damage on diverse middle Palaeocene plants, Menat, France. Proc. R. Soc. B: Biol. Sci. 2009, 276, 4271-4277. [CrossRef] [PubMed] 
5. Dyke, G.; Lindow, B. Taphonomy and abundance of birds from the Lower Eocene Fur Formation of Denmark. Geol. J. 2009, 44, 365-373. [CrossRef]

6. Friedman, M.; Beckett, H.T.; Close, R.A.; Johanson, Z. The English Chalk and London Clay: Two remarkable British bony fish Lagerstätten. Geolog. Soc., London, Special Publ. 2015, 430, 165-200. [CrossRef]

7. Smith, M.E.; Singer, B.; Carroll, A. 40Ar/39Ar geochronology of the Eocene Green River Formation, Wyoming. GSA Bull. 2003, 115, 549-565. [CrossRef]

8. Kirejtshuk, A.G.; Legalov, A.A.; Nel, A. A new genus of the subfamily Apioninae (Coleoptera: Brentidae) from the Lower Eocene Oise amber. Paleontol. J. 2015, 49, 1436-1441. [CrossRef]

9. Wilf, P.; Singer, B.S.; Zamaloa, M.C.; Johnson, K.R.; Cúneo, N.R. Early Eocene 40Ar/39Ar age for the Pampa de Jones plant, frog and insect biota (Huitrera Formation, Neuquén Province, Patagonia, Argentina). Ameghiniana 2010, 47, 207-216. [CrossRef]

10. Mathewes, R.W.; Greenwood, D.R.; Archibald, S.B. Paleoenvironment of the Quilchena flora, British Columbia, during the Early Eocene Climatic Optimum. Can. J. Earth Sci. 2016, 53, 1-17. [CrossRef]

11. Mustoe, G.E. Geologic history of Eocene Stonerose Fossil Beds, Republic, Washington, USA. Geosciences 2015, 5, 243-263. [CrossRef]

12. Lenz, O.K.; Wilde, M.; Mertz, D.F.; Riegel, W. New palynology-based astronomical and revised 40Ar/39Ar ages for the Eocene Maar lake of Messel (Germany). Int. J. Earth. Sci. 2015, 104, 873-889. [CrossRef]

13. West, I.M. The Eocene Cliffs of Bournemouth Dorset. Geology of the Wessex Coast. Available online: www.southampton.ac.uk/ \{\}imw/Bournemouth-Geology.htm (accessed on 9 July 2016).

14. Ring, S.J.; Bocherens, H.; Wings, O.; Rabi, M. Divergent Mammalian body size in a stable Eocene greenhouse climate. Sci. Rep. 2020, 10, 3987. [CrossRef] [PubMed]

15. Sachse, M. A remarkable fossiliferous Mass flow deposit in the Eocene Eckfeld Maar (Germany)-Sedimentological, taphonomical and palaeoecological considerations. Facies 2005, 51, 173-184. [CrossRef]

16. Evanoff, E.; McIntosh, W.C.; Murphey, P.C. Stratigraphic summary and 40Ar/39Ar geochronology of the Florissant Formation, Colorado. Denver Mus. Nat. Sci. Proc. 2001, 4, 1-16.

17. Scott, J.W.; Bowring, S.A. High Precision U/Pb Geochronology of Oligocene Tuffs from the White River Formation, Douglas, Wyoming. J. Vertebr. Paleontol. 2000, 20, 69A.

18. Walther, H.; Kvaček, Z. Early Oligocene flora of Seifhennersdorf (Saxony). Acta Mus. Nat. Pragae, B, Hist. Nat. 2007, 63, 85-174.

19. Schindler, T.; Wuttke, M. Geology and limnology of the Enspel Formation (Chattian, Oligocene; Westerwald, Germany). Palaeobiodiversity Palaeoenvironments 2010, 90, 21-27. [CrossRef]

20. Petrulevičius, J.F.; Wappler, T.; Nel, A.; Rust, J. The diversity of Odonata and their endophytic ovipositions from the Upper Oligocene Fossillagerstätte of Rott (Rhineland, Germany). ZooKeys 2011, 130, 67-89. [CrossRef]

21. Gaudant, J.; Nel, A.; Nury, D.; Véran, M.; Carnevale, G. The uppermost Oligocene of Aix-en-Provence (Bouches-du-Rhône, Southern France): A Cenozoic brackish subtropical Konservat-Lagerstätte, with fishes, insects and plants. C.R.Palevol 2018, 17,460-478. [CrossRef]

22. Seyfullah, L.J.; Beimforde, C.; Dal Corso, J.; Perrichot, V.; Rikkinen, J.; Schmidt, A.R. Production and preservation of resins-Past and present. Biol. Rev. 2018, 93, 1684-1714. [CrossRef]

23. Bukejs, A.; Alekseev, V.I.; Pollock, D.A. Waidelotinae, a new subfamily of Pyrochroidae (Coleoptera: Tenebrionoidea) fromBaltic amber of the Sambian peninsula and the interpretation of Sambian amber stratigraphy, age and location. Zootaxa 2019, 4664, 261-273. [CrossRef] [PubMed]

24. Thompson, R.T. Observations on the morphology and classification of weevils (Coleoptera, Curculionoidea) with a key to Major groups. J. Nat. Hist. 1992, 26, 835-891. [CrossRef]

25. Kuschel, G. A phylogenetic classification of Curculionoidea to families and subfamilies. Mem. Entomol. Soc. Wash. 1995, 14, 5-33.

26. Alonso-Zarazaga, M.A.; Lyal, C.H.C. A world catalogue of families and genera Curculionoidea (Insecta: Coleoptera) (excepting Scolytidae and Platypodidae); Entomopraxis: Barcelona, Spain, 1999; p. 315.

27. Oberprieler, R.G.; Marvaldi, A.E.; Anderson, R.S. Weevils, weevils, weevils everywhere. Zootaxa 2007, 1668, 491-520. [CrossRef] 
28. Oberprieler, R.G.; Anderson, R.S.; Marvaldi, A.E. Curculionoidea Latreille, 1802: Introduction, Phylogeny. In Handbook of Zoology. Arthropoda: Insecta; Coleoptera, Beetles. Volume 3: Morphology and Systematics (Phytophaga); Leschen, R.A.B., Beutel, R.G., Eds.; Walter de Gruyter: Berlin, Germany; New York, NY, USA, 2014; pp. 285-300.

29. Gratshev, V.G.; Legalov, A.A. The Mesozoic stage of evolution of the family Nemonychidae (Coleoptera, Curculionoidea). Paleontol. J. 2014, 48, 851-944. [CrossRef]

30. Legalov, A.A. Fossil Mesozoic and Cenozoic weevils (Coleoptera, Obrienioidea, Curculionoidea). Paleontol. J. 2015, 49, 1442-1513. [CrossRef]

31. Zherichin, V.V.; Gratshev, V.G. Obrieniidae, fam. nov., the oldest Mesozoic weevils (Coleoptera, Curculionoidea). Paleontol. J. 1993, 27, 50-69.

32. Bouchard, P.; Bousquet, Y.; Davies, A.E.; Alonso-Zarazaga, M.A.; Lawrence, J.F.; Lyal, C.H.C.; Newton, A.F.; Reid, C.A.M.; Schmitt, M.; Slipiński, S.A.; et al. Family group names in Coleoptera (Insecta). Zookeys 2001, 88, 1-972. [CrossRef]

33. Legalov, A.A. New Obrieniidae from the Jurassic of Kazakhstan (Coleoptera, Obrienioidea). Paleontol. J. 2012, 46, 73-78. [CrossRef]

34. Wood, S.L. A reclasification of the genera of Scolytidae (Coleoptera). Great Basin Nat. Mem. 1986, 10, 1-126.

35. Sanborne, M. Biology of Ithycerus noveboracensis (Forster) (Coleoptera) and weevil phylogeny. Evol. Monogr. 1981, 4, 1-80.

36. Marvaldi, A.E.; Sequeira, A.S.; O’Brien, C.W.; Farrell, B.D. Molecular and morphological phylogenetics of weevils (Coleoptera: Curculionoidea): Do niche shifts accompany diversification? Syst. biol. 2002, 51, 761-785. [CrossRef] [PubMed]

37. Legalov, A.A. Contribution to the knowledge of the family Nemonychidae (Coleoptera) with descriptions of new taxa. Ukr. J. Ecol. 2017, 7, 64-87. [CrossRef]

38. Legalov, A.A. Annotated key to weevils of the world. Part 1. Families Nemonychidae, Anthribidae, Belidae, Ithyceridae, Rhynchitidae, Brachyceridae and Brentidae. Ukr. J. Ecol. 2018, 8, 780-831. [CrossRef]

39. Legalov, A.A. Annotated key to weevils of the world. Part 2. Subfamily Molytinae (Coleoptera, Curculionidae). Ukr. J. Ecol. 2018, 8, 340-350.

40. Legalov, A.A. Annotated key to weevils of the world. Part 3. Subfamily Conoderinae (Coleoptera, Curculionidae). Ukr. J. Ecol. 2018, 8, 494-503.

41. Legalov, A.A. Annotated key to weevils of the world. Part 4. Subfamilies Erirhininae, Dryophthorinae and Cossoninae (Curculionidae). Ukr. J. Ecol. 2020, 10, 319-331. [CrossRef]

42. Legalov, A.A. Annotated key to weevils of the world. Part 5. Subfamily Entiminae (Curculionidae). Ukr. J. Ecol. 2020, 10, 332-346. [CrossRef]

43. Kuschel, G. The Nearctic Nemonychidae (Coleoptera, Curculionoidea). Entomol. Scand. 1989, 20, 121-171. [CrossRef]

44. Seunggwan, S.; Clarke, D.J.; Lemmon, A.R.; Lemmon, E.M.; Aitken, A.L.; Haddad, S.; Farrell, B.D.; Marvaldi, A.E.; Oberprieler, R.G.; McKenna, D.D. Phylogenomic data yield new and robust insights into the phylogeny and evolution of weevils. Mol. Biol. Evol. 2018, 35, 823-836. [CrossRef]

45. Rheinheimer, J. Illustrierter Katalog und Bibliographie der Anthribidae der Welt (Insecta: Coleoptera). Mitt. Entomol. Ver. Stuttg. 2004, 39, 1-288.

46. Gratshev, V.G.; Legalov, A.A. New Mesozoic Ithyceridae beetles (Coleoptera). Paleontol. J. 2011, 45, 76-81. [CrossRef]

47. Legalov, A.A. New and little known weevils (Coleoptera: Curculionoidea) from the Paleogene and Neogene. Hist. Biol. 2013, 25, 59-80. [CrossRef]

48. Legalov, A.A.; Poinar, G., Jr. New tribes of the superfamily Curculionoidea (Coleoptera) in Burmese amber. Hist. Biol. 2015, 27, 558-564. [CrossRef]

49. Poinar, G., Jr.; Brown, A.E.; Legalov, A.A. A new weevil tribe, Mekorhamphini trib. nov. (Coleoptera, Ithyceridae) with two new genera in Burmese amber. Ukr. J. Ecol. 2016, 6, 157-163. [CrossRef]

50. Legalov, A.A.; Nel, A.; Kirejtshuk, A.G. New and little known weevils (Coleoptera: Curculionoidea) from the Paleocene of Menat (France). C.R. Palevol. 2017, 16, 248-256. [CrossRef]

51. Poinar, G.O., Jr.; Brown, A.E.; Legalov, A.A. A new weevil, Periosomerus tanyorhynchus gen. et sp. nov. (Coleoptera; Ithyceridae) in mid-Cretaceous Burmese amber. Cret. Res. 2019, 104, 104195. [CrossRef] 
52. Zimmerman, E.C. Australian Weevils (Coleoptera. Curculionoidea). I. Anthribidae to Attelabidae; CSIRO Publishing: Melbourne, Australia, 1994.

53. Hamilton, R.W. Taxanomic use of endophallic structures in some Attelabidae and Rhynchitidae of America, North of Mexico (Coleoptera: Curculionoidea), with notes on nomenclature. Ann. Entomol. Soc. America. 1979, 72, 29-34. [CrossRef]

54. Sawada, Y. A systematic study of the family Rhynchitidae of Japan (Coleoptera, Curculionoidea). Hum. Nat. 1993, 2, 1-93.

55. Legalov, A.A. Taxonomy, classification and phylogeny of the leaf-rolling weevils (Coleoptera: Rhynchitidae, Attelabidae) of the world fauna. Novosib. CD-R 2003. № 0320301200.

56. Legalov, A.A. Reconstruction of phylogeny in leaf-rolling weevils (Coleoptera, Rhynchitidae, Attelabidae) using the Synap method. Report 1. Zool. Zhurnal. 2004, 83, 1427-1432.

57. Legalov, A.A. Reconstruction of phylogeny in leaf-rolling weevils (Coleoptera, Rhynchitidae, Attelabidae) using the SYNAP method. Report 2. Zool. Zhurnal. 2005, 84, 190-194.

58. Legalov, A.A. Trophic relations of leaf-rolling weevils (Coleoptera, Rhynchitidae, Attelabidae). Zool. Zhurnal. 2005, 84, 352-361.

59. Legalov, A.A. Phylogenetic reconstruction of weevils superfamily Curculionoidea (Coleoptera) using the SYNAP method. Biol. Bull. 2006, 33, 127-134. [CrossRef]

60. Legalov, A.A. Leaf-Rolling Weevils (Coleoptera: Rhynchitidae, Attelabidae) of the World Fauna; Agro-Siberia: Novosibirsk, Russia, 2007.

61. Legalov, A.A. The family Rhynchitidae (Insecta: Coleoptera) in the Himalayas. Biodiversitäd und Nat. im Himalaya 2015, 5, 479-486.

62. Zimmerman, E.C. Australian Weevils (Coleoptera. Curculionoidea). III. Nanophyidae, Rhynchophoridae, Erirhinidae, Curculionidae: Amycterinae, Literature Consulted; CSIRO Publications: Melbourne, Australia, 1993.

63. Zimmerman, E.C. Australian Weevils (Coleoptera. Curculionoidea). II. Brentidae, Eurhynchidae, Apionidae and a Chapter on Immature Stages by Brenda May; CSIRO Publications: Melbourne, Australia, 1994.

64. Wanat, M. Genera of Australo-Pacific Rhadinocybinae and Myrmacicelinae, with Biogeography of the Apionidae (Coleoptera: Curculionoidea) and Phylogeny of the Brentidae (s. lato); Mantis: Olsztyn, Poland, 2001.

65. Zherikhin, V.V.; Egorov, A.B. Weevils (Coleoptera, Curculionidae) from Russian Far East; Institute Biology Soil Science: Vladivostok, Russia, 1991.

66. Morimoto, K.; Kojima, H. Systematic position of the tribe Phylloplatypodini, with remarks on the definitions of the families Scolytidae, Platypodidae, Dryophthoridae and Curculionidae (Coleoptera: Curculionoidea). Esakia 2004, 44, 153-168.

67. Legalov, A.A. New and little known Apioninae (Coleoptera, Brentidae) in Eocene Baltic amber. Evraz. Entomol. Zhurn. 2012, 11, 219-222.

68. Legalov, A.A. A review of the tribe Auletini (Coleoptera, Rhynchitidae) from the Russian fauna. 2. Subtribe Pseudomesauletina. Zool. Zhurnal 2011, 90, 149-155. [CrossRef]

69. Legalov, A.A. New weevils (Coleoptera, Curculionoidea) from the Eocene of the Green River, United States: Part 1. Paleontol. J. 2018, 52, 294-302. [CrossRef]

70. Legalov, A.A. New weevils (Coleoptera, Curculionoidea) from the Eocene of the Green River, United States: Part 2. Paleontol. J. 2018, 52, 421-428. [CrossRef]

71. Legalov, A.A. A review of the Curculionoidea (Coleoptera) from European Eocene ambers. Geosciences 2020, 10, 16. [CrossRef]

72. Legalov, A.A. A new tribe of the subfamily Cretonemonychinae (Coleoptera, Nemonychidae) from the Middle Eocene of the Green River. Paleontol. J. 2013, 47, 410-413. [CrossRef]

73. Scudder, S.H. Fossil Coleoptera from the Rocky mountain tertiaries. Bull. US Geol. Geogr. Surv. Terr. 1876, 2, 77-87.

74. Scudder, S.H. The fossil insects of the Green River shales. Bull. US Geol. Geogr. Surv. Terr. 1878, 4, 747-776.

75. Riedel, A. A new tribe, genus and species of Nemonychidae from Baltic amber (Coleoptera: Curculionoidea: Nemonychidae: Cimberidinae). Insect Syst. Evol. 2010, 41, 29-38. [CrossRef]

76. Wickham, H.F. Fossil Coleoptera from Florissant, with descriptions of several new species. Bull. Am. Mus. Nat. Hist. 1911, 30, 53-69.

77. Scudder, S.H. Tertiary rhynchophorus Coleoptera of the United States. Monogr. US Geol. Surv. Tert. 1893, 21, 1-206. [CrossRef] 
78. Scudder, S.H. The Tertiary insects of North America. US Geol. Surv. Terr. 1890, 13, 1-734.

79. Zherikhin, V.V. On weevils (Insecta, Coleoptera) from the Baltic amber. Tr. Paleontol. Inst. Akad. Nauk. SSSR. 1971, 130, 197-209.

80. Voss, E. Einige Rhynchophoren der Bernsteinfauna (Col.). Mitt. aus dem Geol. Staatsinst. Hambg. 1953, 22, 119-140.

81. Legalov, A.A.; Kirejtshuk, A.G.; Nel, A. New weevils (Coleoptera, Curculionoidea) from the earlymost Eocene Oise amber. Paleontol. J. 2019, 53, 729-751. [CrossRef]

82. Legalov, A.A. New Curculionoid beetles (Coleoptera: Curculionoidea) from the Baltic amber. Paleontol. J. 2012, 46, 262-272. [CrossRef]

83. Gratshev, V.G.; Perkovsky, E.E. New species of the genus Glaesotropis (Insecta: Coleoptera: Anthribidae) from Rovno amber. Paleontol. J. 2008, 42, 60-63.

84. Gratshev, V.G.; Zherikhin, V.V. A new anthribid genus from the Baltic amber (Insecta: Coleoptera: Anthribidae). Mitt. aus dem Geol. -Paläontologischen Intitut der Univ. Hambg. 1995, 78, 149-157.

85. Heyden, C.; Heyden, L. Käfer und Polypen aus der Braunkohle des Siebengebirges, XXII-XXIV. Palaeontographica 1866, 15, 131-156.

86. Legalov, A.A.; Nazarenko, V.Y.; Perkovsky, E.E. A new genus of fungus weevils (Coleoptera: Anthribidae) in Rovno amber. Foss. Rec. 2018, 21, 207-212. [CrossRef]

87. Foerster, B. Die Insekten des 'Plattigen Steinmergels' von Brunstatt, XI-XVI. Abh. Geol. Spec. Elsass Lotheringen 1891, 3, 333-594.

88. Heyden, C.H.G. Gliederthiere aus der Braunkohle des Niederrhein's, der Wetterau und der Röhn. Palaeontographica 1862, 10, 62-82.

89. Schlechtendal, D.H.R. Beiträge zur Kenntnis Fossiler Insekten aus dem Braunkohlengebirge von Rott am Sie bengebirge, XII-XIV. Abh. Nat. Gesellsch. Halle 1894, 20, 197-228.

90. Legalov, A.A. Two new genera and four new species of fossil weevils (Coleoptera: Curculionoidea) in Baltic amber. Entomol. Fenn. 2016, 27, 57-69. [CrossRef]

91. Legalov, A.A.; Poinar, G.O., Jr. Two new species and new findings of Curculionoidea (Insecta: Coleoptera) in Baltic amber. Ukr. J. Ecol. 2020, 10, 357-364. [CrossRef]

92. Legalov, A.A. A new weevil genus of the tribe Metrioxenini (Coleoptera: Belidae) in Eocene Baltic amber. Hist. Biol. 2012, 24, 213-217. [CrossRef]

93. Riedel, A.; dos Santos Rolo, T.; Cecilia, A.; van de Kamp, T. Sayrevilleinae Legalov, a newly recognised subfamily of fossil weevils (Coleoptera, Curculionoidea, Attelabidae) and the use of synchrotron microtomography to examine inclusions in amber. Zool. J. Linn. Soc. 2012, 165, 773-794. [CrossRef]

94. Kania, J.; Legalov, A.A. A new genus of tooth-nosed snout weevils (Coleoptera: Rhynchitidae) in Baltic amber. Paleontol. J. 2019, 53, 1040-1044. [CrossRef]

95. Bukejs, A.; Legalov, A.A. The first record of Rhynchitidae (Coleoptera) from Rovno amber. Entomol. Fenn. 2019, 30, 168-172. [CrossRef]

96. Wickham, H.F. A report on some recent of fossil Coleoptera from the Miocene Shales of Florissant, I-VIII. Bull. Lab. Nat. Hist. St. Univ. Iowa 1912, 6, 3-38.

97. Wickham, H.F. Fossil Coleoptera from the Wilson Ranch near Florissant, Colorado, I-VII. Bull. Lab. Nat. Hist. State. Univ. Iowa. 1913, 6, 3-29.

98. Zherikhin, V.V. Tertiary weevils (Insecta, Coleoptera: Curculionoidea), identified from the collections of the Senckenberg Museum, Senckenberg. Lethaea 1992, 72, 169-178.

99. Piton, L. Paléontologie du gisement Éocène de Menat (PuydeDôme) (Flore et faune); Imprim. Paul Vallier: ClermontFerrand, France, 1940.

100. Wickham, H.F. New fossil Coleoptera from the Florissant beds Bull. Lab. Nat. Hist. St. Univ. Iowa 1916, 7, 3-19, pls. I-IV.

101. Wickham, H.F. On some fossil Rhynchophorus Coleoptera from Florissant Colorado, I-IV. Bull. Am. Mus. Nat. Hist. 1912, 31, 41-55.

102. Rheinheimer, J. Neue fossile Rüsselkäfer (Coleoptera: Curculionidae) aus dem Eozän des Baltischen Bernsteins und der Grube Messel bei Darmstadt. Staatliches. Mus. Nat. 2007, 365, 1-24.

103. Legalov, A.A. The oldest Brentidae and Curculionidae (Coleoptera: Curculionoidea) from the Aptian of Bon-Tsagaan. Hist. Biol. 2014, 26, 6-15. [CrossRef] 
104. Legalov, A.A. Two new weevil genera of the family Brentidae (Coleoptera) in Baltic amber. Entomol. Fennica 2018, 29, 161-168. [CrossRef]

105. Voss, E. Einige Rüsselkäfer der Tertiärzeit aus baltischen Bernstein (Coleoptera, Curculionidea). Steenstupia 1972, 2, 167-181.

106. Legalov, A.A.; Bukejs, A. Succinapion telnovi n. gen. et n. sp. of the tribe Kalcapiini (Coleoptera: Brentidae: Apioninae) in Baltic amber. Hist. Biol. 2014, 26, 603-607. [CrossRef]

107. Wagner, H. Ein neues Apion aus dem baltischen Bernstein (Coleoptera, Curculionidae). Deut. Entomol. Zeitsch. 1924, 1924, 134-136.

108. Bukejs, A.; Legalov, A.A. The first record of Brentidae (Coleoptera) in Eocene Rovno amber with description of a new fossil species of Toxorhynchus Scudder, 1893. Fossil Record 2020, 23, 169-177. [CrossRef]

109. Legalov, A.A. New weevils of the family Brentidae (Coleoptera) in Baltic amber. Paleontol. J.. (In Press).

110. Oustalet, M.E. Recherches sur les insectes fossiles des terraines tertiaires de la France. 2 me partie. Insectes fossiles d'Aix en Provence. Ann. Sci. Géol. Paris 1874, 5, 1-347.

111. Legalov, A.A. First record of the weevil subfamily Nanophyinae (Coleoptera, Brentidae) from the Eocene of the Green River, United States. Paleontol. J. 2015, 49, 399-401. [CrossRef]

112. Théobald, N. Les insectes fossiles des terrains oligocènes de France. Bull. Mensuel. Soc. Sci. Nancy 1937, $1,1-473$.

113. Legalov, A.A.; Wappler, T. The oldest record of straight-snouted weevils (Coleoptera: Curculionoidea: Brentidae: Brentinae) from the Eocene of Germany. Hist. Biol. 2019, 1-9. [CrossRef]

114. Britton, E.B. Beetles from the London clay (Eocene) of Bognor regis, Sussex. Bull. Br. Mus. Nat. Hist. Geol. 1960, 4, 27-50.

115. Legalov, A.A. New weevils (Curculionidae) in Baltic amber. Paleontol. J. 2016, 50, 970-985. [CrossRef]

116. Bukejs, A.; Legalov, A.A. A new species of the genus Dorytomus Germar, 1817 (Coleoptera, Curculionidae) from Baltic amber. Entomol. Fenn. 2019, 30, 173-178. [CrossRef]

117. Legalov, A.A.; Nazarenko, V.Y.; Perkovsky, E.E. New weevils (Coleoptera: Curculionidae) from Rovno amber. Paleontol. J. 2019, 53, 1045-1059. [CrossRef]

118. Legalov, A.A. Checklist of Mesozoic Curculionoidea (Coleoptera) with description of new taxa. Balt. J. Col. 2010, 10, 71-101.

119. Oustalet, F.E. Recherches sur les insectes fossiles des terraines tertiaires de la France. Ann. Sci. Géol. Paris 1870, 2, 1-178.

120. Beier, M. Miozäne und oligozäne Insekten aus Österreich und den unmitulbar angrenzenden Gebieten. Sitzungsberichte Österreichische Akademie der Wissenschaften, Mathe MatischenNaturwissensschaftliche Klasse, Abteilung 1: Biologie, Mineralogie, Erdkunde und vewandte Wissenschaften 1952, 161, 129-134.

121. Hustache, A. Un curculionide de l'ambre de la Baltique. Bull. Mens. Soc. Lin. Lyon. 1942, 11, 108-109. [CrossRef]

122. Ulke, T. A new genus and species of Curculionidae (Coleoptera) in Baltic amber. Not. Nat. 1947, 19, 1-5.

123. Cockerell, T.D.A. Some Eocene insects from Colorado and Wyoming. Proc. Unit. Stat. Nat. Mus. 1921, 59, 29-39. [CrossRef]

124. Haupt, H. Die Käfer (Coleoptera) aus der Eozänen Braunkohle des Geiseltales. Geologica 1950, 6, 1-168.

125. Bukejs, A.; Alekseev, V.I.; Legalov, A.A. A new Eocene genus of the subtribe Tylodina (Coleoptera: Curculionidae) and notes concerning local differences of Baltic amber in the Kaliningrad Region. Foss. Rec. 2020, 23, 75-81. [CrossRef]

126. Deichmueller, J.V. Fossile Insecten aus dem Diatomeenschiefir von Kutschlin bei Bilin, Böhmen. Nova Acta Leopold. Verh. Kais. Leopold.Carol. Dtsch. Akad. Nat. 1881, 42, 293-331.

127. Meunier, F. Sur quelques nouveaux insectes des lignites oligocenès (Aquitanien) de Rott, Siebengebirge (Rhénanie). Konin. Akad. Wetensch. Amst. 1924, 26, 605-612.

128. Heyden, C.H.G. Fossile Insekten aus der Braunkohle der Sieblos. Palaeontographica 1858, 5, 115-120.

129. Nazarenko, V.Y.; Perkovsky, E.E. A new genus and species of Dryophthorid weevils (Coleoptera, Dryophthoridae: Stromboscerinae) from the Rovno amber. Paleontol. J. 2009, 43, 1097-1100. [CrossRef]

130. Piton, L.; Theobald, N. La faune entomologique des gisements miopliocènes du Massif Central. Rev. Sci. Nat. Auvergne 1935, 2, 1-81.

131. Cockerell, T.D.A. Some tertiary fossil insects. An. Mag. Nat. Hist. 1926, 18, 313-324. [CrossRef] 
132. Kuska, A. Three new species of beetles (Coleoptera: Cantharidae, Anobiidae, Curculionidae) from the Baltic amber. Ann. Up. Sil. Mus. Bytom Entomol. 1992, 3, 107-113.

133. Nazarenko, V.Y.; Legalov, A.A.; Perkovsky, E.E. A new species of the genus Caulophilus Woll. (Coleoptera: Curculionidae: Cossoninae) from the Rovno amber. Paleontol. J. 2011, 45, 287-290. [CrossRef]

134. Cockerell, T.D.A. Tertiary insects from Argentina, I. Proc. Unit. Stat. Nat. Mus. 1925, 68, 1-5. [CrossRef]

135. Meunier, F. Über einige fossile Insekten aus den Braunkohlenschichten (Aquitanien) von Rott (Siebengebirge). Z. Deut. Geol. Gesellsch. 1916, 67, 205-225.

136. Wickham, H.F. New species of fossil beetles from Floris sant, Colorado. Proc. US Nat. Mus. 1917, 52, 463-472. [CrossRef]

137. Cockerell, T.D.A. Fossil arthropods in the British Museum. II. An. Mag. Nat. Hist. 1920, 9, 273-279. [CrossRef]

138. Legalov, A.A.; Bukejs, A. A new genus of the tribe Ceutorhynchini (Coleoptera: Curculionidae) in Baltic amber. Entomol. Fenn. 2018, 29, 185-190. [CrossRef]

139. Kuska, A. New beetle species (Coleoptera, Cantharidae, Curculionidae) from the Baltic amber. Pr. Muz. Ziemi 1996, 44, 13-23.

140. Legalov, A.A. A new tribe Palaeoanoplini trib. nov. (Coleoptera: Curculionidae) in Baltic amber. Paleontol. J.. (in press).

141. Legalov, A.A. A new genus of the tribe Curculionini (Coleoptera: Curculionidae) from Baltic amber. Paleontol. J. 2019, 53, 1036-1039. [CrossRef]

142. Zherikhin, V.V. A weevil of the tribe Curculionini (Insecta: Coleoptera, Curculionidae) from the Paleogene of Havighorst, Germany. Mitt. Geol.-Paläontol. Inst. Hambg. 1995, 78, 145-148.

143. Cockerell, T.D.A. New species of North American fossil beetles, cockroaches and tsetse flies. Proc. Unit. Stat. Nat. Mus. 1918, 54, 301-311, pls. 54-55. [CrossRef]

144. Bukejs, A.; Legalov, A.A. Groehnius, a new genus of Eugnomini (Coleoptera: Curculionidae) from Eocene Baltic amber. Foss. Rec. 2019, 22, 45-49. [CrossRef]

145. Nazarenko, V.Y.; Perkovsky, E.E. A new species of derelomine weevils (Coleoptera, Curculionidae, Curculioninae: Acalyptini) from the Rovno amber. Paleontol. J. 2016, 50, 991-996. [CrossRef]

146. Heer, O. Ueber die fossilen Insecten von Aix in der Provence. Vierteljahr. Naturforsch. Gesllschaft. Zürich 1856, 5, 1-40.

147. Germar, E.F. Ueber einige Insekten aus Tertiärgebildungen. Z. Deut. Geol. Gesellsch. 1849, 1, 52-66. [CrossRef]

148. Nicolas, M. Insectes fossiles d'Aix (Provence). Descriptions de Quelques Nouvelles Espèces (Collection de M. Matheron). Assoc. Fr. pour l'Avancement Sci. 1891, 2, 425-438.

149. Giebel, C.G.A. Fauna der Vorwelt mit steter Berücksichtigung der lebenden Thiere. Insecten und Spinnen. Monogr. Dargestellt 1856, 2, 1-511.

150. Zherikhin, V.V. Oligocene seed beetles and weevils (Coleoptera: Bruchidae, Curculionidae) from Bol'shaya Svetlovodnaja River (northern Primorie). In Kainozoi Dal'nego Vost. (Cenozoic of the Far East); Institute Biology Soil Science: Vladivostok, Russia, 1989; pp. 145-150.

151. Legalov, A.A.; Bukejs, A. A new species of the genus Archaeocallirhopalus (Coleoptera: Curculionidae) in Baltic amber. Entomol. Fenn. 2015, 26, 25-29. [CrossRef]

152. Haupt, H. Beitrag zur Kenntnis der Eozänen Arthropodenfauna des Gieseltales. Nova Acta Leopoldiana 1956, 18, 1-90.

153. Wanat, M.; Borovec, L. New genus of weevil (Coleoptera, Curculionidae) from Baltic amber. Pol. Pismo Entomol. 1986, 56, 243-247.

154. Yunakov, N.N.; Kirejtshuk, A.G. New genus and species of broad-nosed weevils from Baltic amber and notes on fossils of the subfamily Entiminae (Coleoptera, Curculionidae). ZooKeys 2011, 160, 73-96. [CrossRef] [PubMed]

155. Bukejs, A.; Legalov, A.A. First record of the tribe Naupactini (Coleoptera: Curculionidae) in Rovno amber. Foss. Rec. 2019, 22, 25-30. [CrossRef]

156. Wickham, H.F. New Miocene Coleoptera from Florissant. Bull. Mus. Compar. Zool. 1914, 58, 423-494, pls. 1-16.

157. Germar, E.F. Fauna Insectorum europae. 19. Insectorum Protogaea Specimen Systems Insecta Carbonum Fossilum; Kuemmelii: Halae, Germany, 1837; Available online: http://dl.ub.uni-freiburg.de/diglit/germar1837 (accessed on 6 September 2020). 
158. Williams, M.Y. The stratigraphy and palaeontology of Hong Kong and the new territories. Trans. Royal. Soc. Can. 1943, 3, 93-116.

159. Cockerell, T.D.A. The fauna of the Sunchal (or Margas Verdes) Formation, Northern Argentina. Am. Mus. Nov. 1936, 886, 1-9.

160. Wickham, H.F. Coleoptera from the Lower Eocene (Wilcox) clays. J. Wash. Acad. Sci. 1929, 19, 148-150.

161. Heer, O. Die miocene Flora and Fauna Spitsbergens, I-XVI. Kongl. Sven. Vetensk.-Akad. Handl. 1870, 8, 1-98.

162. Cockerell, T.D.A. Fossil arthropods in the British Museum. III. An. Mag. Nat. Hist. 1920, 9, 65-72. [CrossRef]

163. Cockerell, T.D.A. Some American fossil Insects. Proc. Unit. Stat. Nat. Mus. 1917, 51, 89-106. [CrossRef]

164. Piton, L.; Rudel, M.A. Sur de nouveaux gisemente d'Insectes fossiles dans l'Oligocene de la Limagne. Bull. Mens. Soc. Linn. Lyon 1936, 5, 78-81.

165. Cockerell, T.D.A. Eocene fossils from Green River, Wioming. Am. J. Sci. 1909, 28, 447-448. [CrossRef]

166. Piton, L.; Theobald, N. Les insectes fossiles du PuydeMur (Limagne). Bull. Menseul. Soc. Sci. Nancy 1937, 9, 202-214.

167. Peris, D.; Solórzano Kraemer, M.M.; Smith, S.M.; Cognato, A.I. Eoplatypus jordali gen.n. et sp.n., the first described Platypodinae (Coleoptera: Curculionidae) from Baltic amber. Arthropod Syst. Phyl. 2017, 75, 85-194.

168. Schedl, K. Die Borkenkäfer des baltischen Bernsteins. Zent. Ges. Entomol. 1947, 2, 12-45.

169. Hagedorn, M. Borkenkäfer des baltischen Bersteins. Schr. Phys.-Ökonom. Gesell. König. Prus. 1906, 47, 115-121.

170. Germar, E.F. Insekten in Bernstein eingeschlossen, beschrieben aus dem academischen Mineralien Cabinet zu Halle. Mag. Entomol. 1813, 1, 11-18.

171. Petrov, A.V.; Zherikhin, V.V. A new species of bark beetles of the genus Hylesinus (Insecta: Coleoptera: Scolytidae) from the Oligocene of France. Paleontol. J. 2004, 38, 187-189.

172. Petrov, A.V.; Perkovsky, E.E. New species of bark beetles from the Rovno amber (Insecta: Coleoptera: Scolytidae). Paleontol. J. 2008, 42, 406-408. [CrossRef]

173. Petrov, A.V.; Perkovsky, E.E. A new genus and species of Scolytinae (Coleoptera: Curculionidae) from the Rovno amber. Paleontol. J. 2018, 52, 164-167. [CrossRef]

174. Martins-Neto, R.G. Review of some Insecta from Mesozoic and Cenozoic Brazilian deposits with descriptions of new taxa. Acta Geol. Leop. 2001, 24, 115-124.

175. Motschulsky, V. Voyages. Lettres de M. de Motschulsky a M. Ménétriés, 4. Étud. Entomol. 1857, 5, 21-38.

176. Lin, Q.B.; Lee, C.M. Early Tertiary insects from Peng Chau, Hong Kong. Palaeontol. Strat. Hong Kong 1997, 1, 185-199.

177. Legalov, A.A. Fossil history of Mesozoic weevils (Coleoptera: Curculionoidea). Insect Sci. 2012, 19, 683-698. [CrossRef]

178. Kuschel, G. Reappraisal of the Baltic Amber Curculionoidea described by E. Voss. Mitt. Geol.-Paläontol. Inst. Hambg. 1992, 73, 191-215.

179. Legalov, A.A. A review of fossil and recent species of the family Ithyceridae (Coleoptera) from the world fauna, I-IV. Amur. Zool. Zh. 2009, 1, 117-131.

180. Legalov, A.A. A new classification of ecological groups of the leaf-rolling weevils (Coleoptera: Rhynchitidae, Attelabidae). Evraz. Entomol. Zhur. 2004, 3, 43-45.

181. Caldara, R.; Franz, N.M.; Oberprieler, R.G. Chapter 2. Curculioninae Latreille 1802. In Handbook of Zoology. Arthropoda: Insecta. Coleoptera, Beetles; Volume 3: Morphology and systematics (Phytophaga); Kristensen, N.P., Beutel, R.G., Eds.; Walter de Gruyter: Berlin, Germany; New York, NY, USA, 2014; Volume 3, pp. 589-628.

182. Meregalli, M. 3.7.6. Lixinae Schoenherr 1823. In Handbook of Zoology. Arthropoda: Insecta. Coleoptera, Beetles; Volume 3: Morphology and Systematics (Phytophaga); Kristensen, N.P., Beutel, R.G., Eds.; Walter de Gruyter: Berlin, Germany; New York, NY, USA, 2014; Volume 3, pp. 523-528.

183. Alonso-Zarazaga, M.A. Revision of the supraspecific taxa in the Palaearctic Apionidae Schoenherr 1823 (Coleoptera, Curculionoidea). 2. Subfamily Apioninae Schoenherr, 1823: Introduction, keys and descriptions. Graellsia 1990, 46, 19-156.

184. Gruas-Cavagnetto, C. Etude palynologique del'Eocene du Bassin Anglo-Parisien. Mem. Soc. Geol. Fr. 1978, $56,1-64$.

185. Archibald, S.B.; Mathewes, R.W. Early Eocene insects from Quilchena, British Columbia and their paleoclimatic implications. Can. J. Zool. 2000, 78, 1441-1462. [CrossRef] 
186. Gratshev, V.G.; Zherikhin, V.V. The fossil record of weevils and related beetle families (Coleoptera, Curculionoidea). Acta Zool. Cracov. 2003, 46, 129-138.

187. Wilf, P.; Donovan, M.P.; Cúneo, N.R.; Gandolfo, M.A. The fossil flip-leaves (Retrophyllum, Podocarpaceae) of southern South America. Am. J. Bot. 2017, 104, 1344-1369. [CrossRef] [PubMed]

188. Lyal, C.H.C. 3.7.7. Molytinae Schoenherr 1823. In Handbook of Zoology. Arthropoda: Insecta. Coleoptera, Beetles; Volume 3: Morphology and Systematics (Phytophaga); Kristensen, N.P., Beutel, R.G., Eds.; Walter de Gruyter: Berlin, Germany; New York, NY, USA, 2014; Volume 3, pp. 529-569.

189. Klebs, R. Über Bernsteinschlüsse im allgemein und die Coleopteren meiner Bernsteinsammlung. Schr. Phys.-ökonom. Gesellsch. Königsberg in Pr. 1910, 51, 217-242.

190. Kirejtshuk, A.G.; Nel, A. Current knowledge of Coleoptera (Insecta) from the lowermost Eocene Oise amber. Insect Syst. Evol. 2013, 44, 175-201. [CrossRef]

191. Alekseev, V.I. Coleoptera from the middle-upper Eocene European ambers: Generic composition, zoogeography and climatic implications. Zootaxa 2017, 4290, 401-443. [CrossRef]

192. O'Brien, C.W.; Tang, W. Revision of the New World cycad weevils of the subtribe Allocorynina, with description of two new genera and three new subgenera (Coleoptera: Belidae: Oxycoryninae). Zootaxa 2015, 3970, 1-87. [CrossRef] [PubMed]

193. Poinar, G.J.; Legalov, A.A. Pleurambus strongylus n. gen., n. sp. (Coleoptera: Belidae) in Dominican amber. Hist. Biol. 2014, 26, 670-674. [CrossRef]

194. Hassan, M.Y. Tertiary faunas from Kap Brewster, East Greenland. Medd. om Grønland 1953, 111, 1-42.

195. Lutz, H. Beitrag zur Kenntnis der unteroligozänen Insektenfauna von Cereste (Süd-Frankreich). Doc. Nat. 1984, 21, 1-26.

196. Prokop, J.; Fikacek, M. Early Oligocene insect fauna from Seifhennersdorf (Saxony, Germany). Acta Mus. Nat. Pragae 2007, 63, 205-213.

197. Cifuentes-Ruiz, P.; Vega, F.J.; Cevallos Ferriz, S.R.S.; Gonzalez-Soriano, E.; Zaragoza Caballero, S.; Garibayromero, L. Oligocene Scorpion and insects (Plecoptera and Coleoptera) from the Los Ahuehuetes Locality, Puebla, Mexico. Ameghiniana 2007, 44, 673-679.

198. Prokop, J. Remarks on palaeoenviromental changes based on reviewed Tertiary insect associations from the Krušnéhory piedmont basins and the Ėeské støedohoøí Mts in northwestern Bohemia (Czech Republic). Acta Zool. Cracov. 2003, 46, 329-344.

199. Wedmann, S.; Poschmann, M.; Hörnschemeyer, T. Fossil insects from the Late Oligocene Enspel Lagerstätte and their palaeobiogeographic and palaeoclimatic significance. Palaeobio. Palaeoenv. 2010, 90, 49-58. [CrossRef]

200. Kirejtshuk, A.G.; Ponomarenko, A.G.; Kurochkin, A.S.; Alexeev, A.V.; Gratshev, V.G.; Solodovnikov, A.V.; Krell, F.-T.; Soriano, C. The beetle (Coleoptera) fauna of the Insect Limestone (late Eocene), Isle of Wight, southern England. Earth Env. Sci. Trans. R. Soc. Edinb. 2019, 110, 405-492. [CrossRef] 